Aus der Klinik für Kardiologie und Pneumologie

(Prof. Dr. med. G. Hasenfuß)

im Zentrum Innere Medizin

der Medizinischen Fakultät der Universität Göttingen

\title{
Prädiktoren von Schocks und Mortalität - Eine klinische Analyse bei Patienten mit implantierbarem Kardioverter-Defibrillator (ICD)
}

\author{
INAUGURAL - DISSERTATION \\ zur Erlangung des Doktorgrades \\ der Medizinischen Fakultät der \\ Georg-August-Universität zu Göttingen
}

vorgelegt von
Hannes Wenk

aus

Rostock

Göttingen 2015 
Dekan:

Prof. Dr. rer. nat. H. K. Kroemer

I. Berichterstatter: Prof. Dr. med. M. Zabel

II. Berichterstatter: PD Dr. Stephan Kazmeier

III. Berichterstatterin: Prof. Dr. Margarete Schön 


\section{Inhaltsverzeichnis}

1. Einleitung.

2. Patienten und Methoden................................................................................................... 6 -

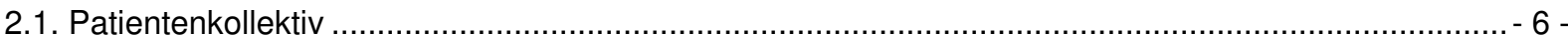

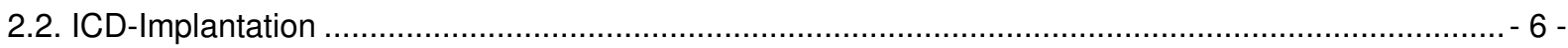

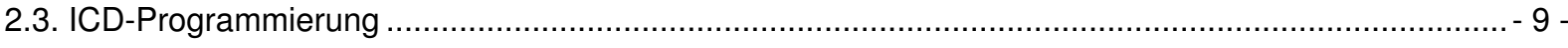

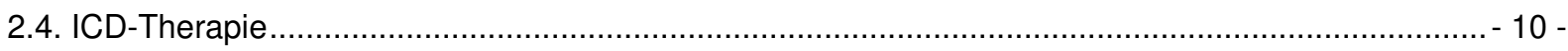

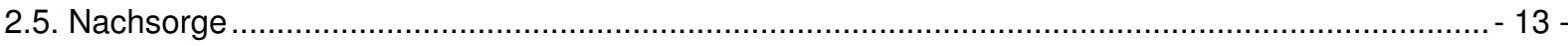

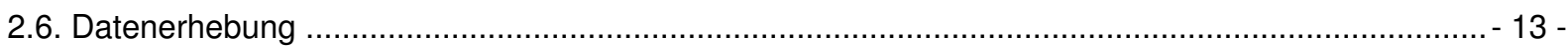

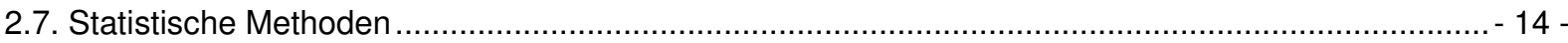

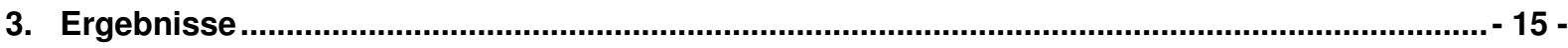

3.1. Charakterisierung des Patientenkollektivs ................................................................... $15-$

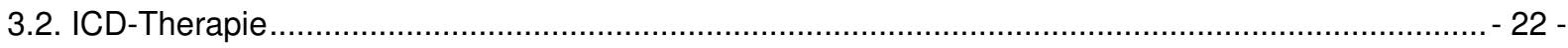

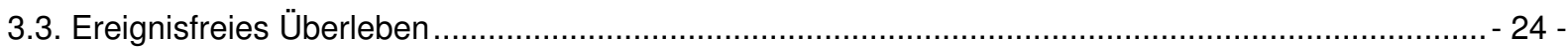

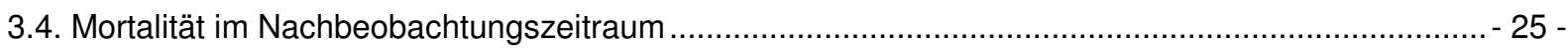

3.5. Analyse von Prädiktoren für appropriate ICD-Schocks ..................................................... 26 -

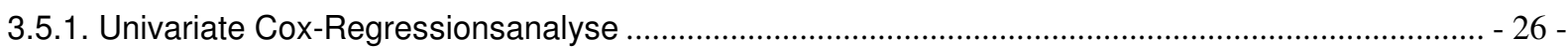

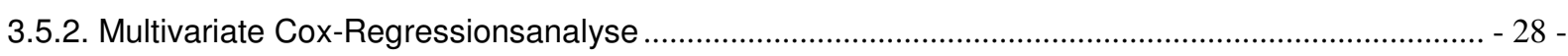

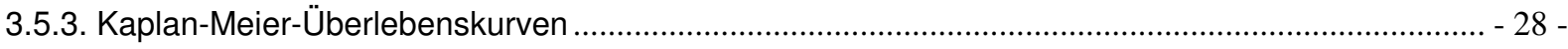

3.6. Analyse von Prädiktoren für inappropriate ICD-Schocks ........................................................... 30 -

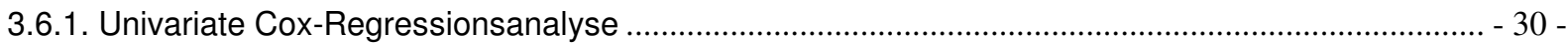

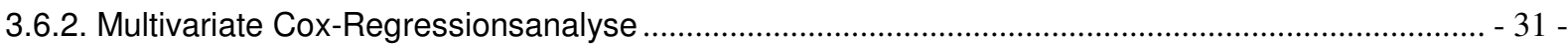

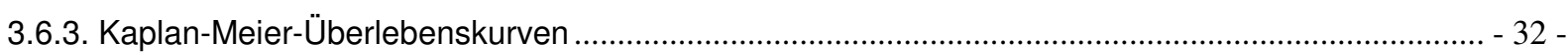

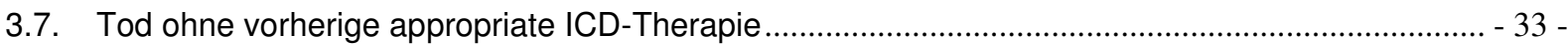

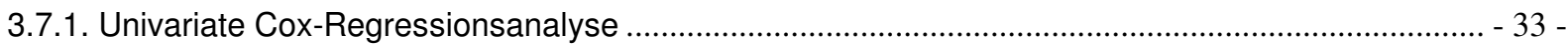

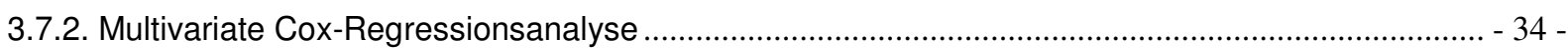

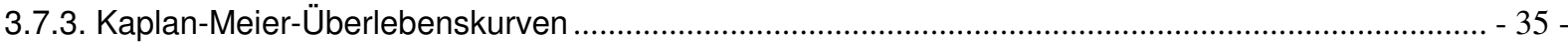

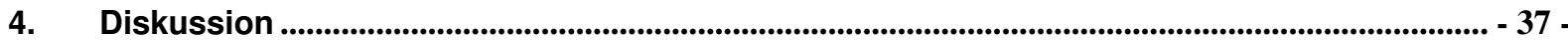

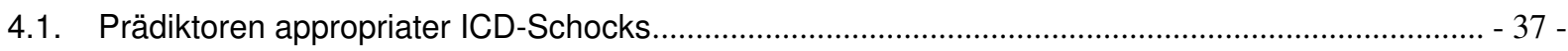

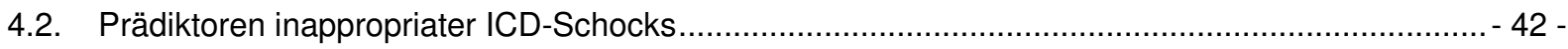

4.3. Prädiktoren für Tod ohne vorherige appropriate ICD-Therapie ....................................... 48 -

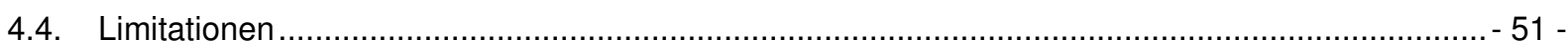

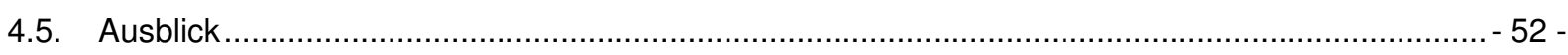

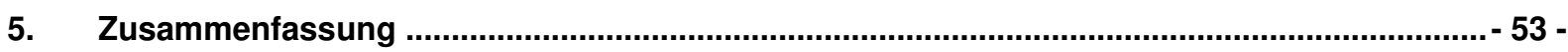

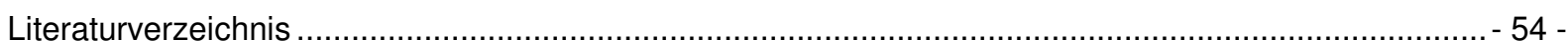

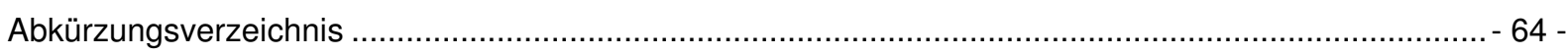

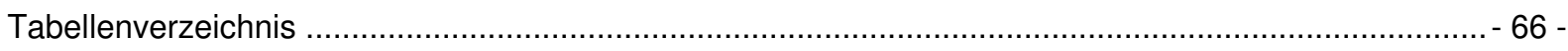

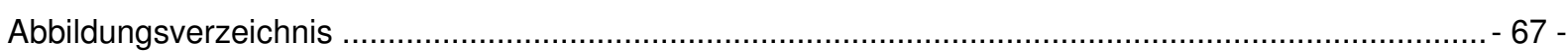




\section{Einleitung}

Der plötzliche Herztod ist definiert als Tod aufgrund von unerwartetem Kreislaufstillstand wegen kardialer Arrhythmien, der innerhalb einer Stunde nach Beginn der Symptome auftritt (Zipes et al. 2006).

Die Pathophysiologie der diese Situation herbeiführenden Vorgänge ist komplex. Auf dem Boden einer strukturellen Herzerkrankung können verschiedene Faktoren eine elektrische Instabilität mit nachfolgenden ventrikulären Arrhythmien verursachen, die schließlich zu einem Herz-Kreislauf-Stillstand führen können.

Es gibt zahlreiche kardiale Grunderkrankungen, die das Auftreten eines plötzlichen Herztodes begünstigen, am häufigsten sind dies eine koronare Herzkrankheit (KHK) und nicht ischämische, dilatative Kardiomyopathien (DCM), seltener valvuläre Herzkrankheiten, das Long-QT-Syndrom, das Brugada-Syndrom oder eine rechtsventrikuläre Dysplasie. Mit höherem Lebensalter nimmt die Inzidenz des plötzlichen Herztodes zu, bei 50 Jahre alten Männern beträgt sie 100 je 100.000, bei 75-Jährigen schon 800 pro 100.000, während Frauen im Allgemeinen seltener betroffen sind (Deo und Albert 2012). Ein besonderer Umstand ist, dass mehr als die Hälfte der betroffenen Patienten keine bekannte kardiale Grunderkrankung aufweisen, bei Frauen liegt die Rate sogar bei 69\% (Albert et al. 2003). Das heißt, der plötzliche Herztod ist bei diesen Patienten die klinische Erstmanifestation einer kardialen Erkrankung.

Die Mehrzahl der Fälle tritt im außerklinischen Umfeld oder unbeobachtet auf. Auch nach notfallmäßiger Behandlung durch geschultes Personal sind die Überlebensraten gering. Nichol et al. (2008) geben diese nach einer Untersuchung von 20.520 Fällen von plötzlichem Herztod mit circa $8 \%$ an.

Insgesamt zählt der plötzliche Herztod zu den häufigsten Todesursachen und macht etwa 15-20\% aller Todesfälle aus (Deo und Albert 2012). Die Inzidenz in Deutschland beträgt schätzungsweise 70.000 - 100.000 Fälle pro Jahr (Trappe 2012). Der überwiegende Anteil wird dabei durch ventrikuläre Tachyarrhythmien (ventrikuläre Tachykardien oder Kammerflimmern) ausgelöst. Nur ein geringer Anteil von circa $15 \%$ entsteht auf dem Boden bradykarder Herzrhythmusstörungen, meist als Zeichen einer Herzinsuffizienz im Endstadium und elektromechanischer Dissoziation (Josephson und Wellens 2004). Zur Kontrolle dieser Rhythmusstörungen hat sich neben einer medikamentösen antiarrhythmischen Therapie vor allem die Behandlung mit einem automatischen implantierbaren Kardioverter-Defibrillator (ICD) als wichtigstes Instrument zur Prävention des plötzlichen Herztodes entwickelt. Der Vorteil ist die 
unmittelbar erfolgende Therapie, im Gegensatz zur oft beträchtlichen zeitlichen Verzögerung bis zur externen Defibrillation. Seit der ersten Implantation im Jahre 1980 in den USA erfolgte eine kontinuierliche technische Weiterentwicklung. Waren die ersten Geräte noch relativ groß und wurden abdominal platziert, so ist der heutige Standard eine pektorale Implantation mit transvenösen Sonden zur Detektion und kontinuierlichen Aufzeichnung des Herzrhythmus sowie eventuell nötig werdender Stimulation und Defibrillation.

Entsprechend den über die Zeit angepassten Leitlinien (Epstein et al. 2008, Dickstein et al. 2010, Epstein et al. 2013) veränderten sich die Empfehlungen zu ICDImplantationen. In der letztgenannten Version besteht für folgende Patientenkollektive eine Klasse-I-Indikation zur ICD-Implantation: Patienten nach überlebtem plötzlichem Herztod durch Kammerflimmern (VF), anhaltende ventrikuläre Tachykardie (VT) oder Synkope unklarer Genese mit zusätzlicher Induzierbarkeit ventrikulärer Tachyarrhythmien in einer elektrophysiologischen Untersuchung (EPU). Dies wird als Sekundärprophylaxe des plötzlichen Herztodes bezeichnet. Patienten, die bisher noch keine lebensbedrohlichen, ventrikulären Tachyarrhythmien erlitten haben, aber ein hohes Risiko für Rhythmusereignisse aufweisen, erhalten den ICD zur sogenannten Primärprophylaxe des plötzlichen Herztodes. Dies sind Patienten mit Zustand nach Myokardinfarkt (mehr als 40 Tage) und entweder einer linksventrikulären Ejektionsfraktion (LVEF) $\leq 30 \%$ und Herzinsuffizienz entsprechend New York Heart Association (NYHA) Stadium I oder einer LVEF $\leq 35 \%$ bei NYHA-Stadium II oder III. Patienten mit nicht anhaltenden VT nach Myokardinfarkt und einer LVEF $\leq 40 \%$ müssen zusätzlich induzierbare ventrikuläre Tachyarrhythmien in einer EPU aufweisen. Bei Vorliegen einer nicht-ischämischen dilatativen Kardiomyopathie und einer LVEF $\leq 35 \%$ muss für eine Klasse-I-Indikation ein NYHA-Stadium II oder III vorliegen (Epstein et al. 2013).

Die genannten Empfehlungen basieren auf den Ergebnissen zahlreicher klinischer Studien, die im Folgenden kurz skizziert werden sollen. Gemeinsam ist diesen die Erkenntnis, dass ein ICD nicht nur zuverlässig Tachyarrhythmien terminiert, sondern verglichen mit einer konservativen, meist medikamentösen, Therapie auch das Gesamtüberleben positiv beeinflusst. In den nicht randomisierten Studien AVID (Antiarrhythmics versus Implantable Defibrillators), CASH (Cardiac Arrest Study Hamburg) und CIDS (Canadian implantable defibrillator study) konnte eine Reduktion 
der Gesamtmortalität nach sekundärprophylaktischer ICD-Implantation gezeigt werden, allerdings wurde in den beiden letztgenannten Untersuchungen keine statistische Signifikanz erreicht (The AVID-Investigators 1997, Kuck et al. 2000, Connolly et al. 2000). Die erste randomisierte Studie zum Nutzen einer primärprophylaktischen ICD-Implantation war MADIT (Multicenter Automatic Defibrillator Implantation Trial). Es zeigte sich eine signifikante Reduktion der Gesamtmortalität um 54\% im Vergleich von ICD zu alleiniger medikamentöser antiarrhythmischer Therapie (Moss et al. 1996). In der MUSTT-Studie (Multicenter Unsustained Tachycardia Trial) betrug die Mortalitätsreduktion durch primärprophylaktisch implantierten ICD gegenüber antiarrhythmischer Medikation sogar 60\%. Bemerkenswert war die fehlende Überlegenheit einer medikamentösen Therapie gegenüber keiner Therapie in Bezug auf plötzlichen Herztod oder Gesamtmortalität. Der Nutzen von elektro-physiologischen Testungen, um über die Art der Therapie zu entscheiden, konnte nicht bestätigt werden (Buxton et al. 1999). MADIT-II (Multicenter Automatic Defibrillator Implantation Trial II) bestätigte ebenfalls eine signifikante Reduktion des Mortalitätsrisikos um 31\% in der ICDGruppe gegenüber konventioneller Therapie bei primärprophylaktischen Einschlusskriterien (Moss et al. 2002). Eine Mortalitäts-reduktion durch eine ICD-Implantation nach kürzlich stattgehabtem Myokardinfarkt (6-40 Tage zuvor) konnte in der DINAMIT-Studie (Defibrillator in Acute Myocardial Infarction Trial) nicht bestätigt werden, da die Reduktion arrhythmisch bedingter Todesfälle durch einen Anstieg anderer Todesursachen in der ICD-Gruppe aufgehoben wurde (Hohnloser et al. 2004). Der Nutzen einer ICD-Implantation für Patienten mit nicht-ischämischer DCM wurde in der DEFINITE-Studie (Defibrillators in Non-Ischemic Cardiomyopathy Treatment Evaluation) randomisiert untersucht. Trotz einer 80-prozentigen Reduktion von plötzlichem Herztod war die Senkung der Gesamtmortalität statistisch nicht signifikant (Kadish et al. 2004). Hingegen zeigte sich eine Signifikanz für die 23-prozentige Mortalitätsreduktion durch einen Einkammer-ICD verglichen mit Amiodaron oder Placebo in der SCD-HeFT-Studie (Sudden Cardiac Death in Heart Failure Trial) bei je etwa zur Hälfte ischämisch und nicht-ischämisch bedingter Herzinsuffizienz (Bardy et al. 2005). Die Senkung der Gesamtmortalität um 36\% durch biventrikuläre Stimulation mit zusätzlichem ICD zeigte die Studie COMPANION (Comparison of Medical Therapy, Pacing, and Defibrillation in Heart Failure) bei Patienten mit fortgeschrittener Herzinsuffizienz entsprechend NYHA-Stadium III oder IV (Bristow et al. 2004). In der MADIT-CRT-Studie (Multicenter Automatic Defibrillator Implantation Trial with Cardiac Resynchronization Therapy) bei Patienten mit milderer Herzinsuffizienz 
(NYHA-Stadium I oder II) und LVEF $\leq 30 \%$ konnte zwar eine 41-prozentige Reduktion von Herzversagen und eine signifikant verbesserte LVEF durch einen CRT-D im Vergleich zu alleinigem ICD gezeigt werden, eine Überlegenheit in Bezug auf die Gesamtmortalität war allerdings nicht nachweisbar (Moss et al. 2009).

Aufgrund der zunehmenden Bedeutung der Primärprophylaxe wurde ein starker Anstieg der Implantationszahlen beobachtet. Aus diesem Grund wird die Kosteneffektivität der ICD-Therapie zunehmend wichtiger. Smith et al. (2012) gaben in einer Metaanalyse klinischer Studien zur primärprophylaktischen ICD-Implantation die Kosten je gewonnenem Lebensjahr und angepasst an die Lebensqualität und Morbidität (QALY, quality adjusted life year) mit durchschnittlich 43.993 Euro je QALY an und schlussfolgtern, dass die ICD-Therapie bei einer Grenze von 80.000 Euro je QALY kosteneffektiv sei. Besonders galt dies für Patienten, die ein hohes Risiko für einen arrhythmisch bedingten Tod und ein geringes Risiko für einen Tod anderer Ursache hatten. Kosteneffektiv sei die ICD-Therapie laut Goldenberg et al. (2005) auch für Hochrisiko-Patienten mit Long-QT-Syndrom oder hypertropher Kardiomyopathie, die im Allgemeinen jünger sind und bei denen eine höhere Lebenserwartung unter anderem zu einer höheren Produktivität führt.

Die Gesamtkosten für ICD bei allen gesetzlich versicherten Patienten in Deutschland, die die MADIT-II-Kriterien (Zustand nach Myokardinfarkt und LVEF $\leq 30 \%$ ) erfüllen, belaufen sich nach Berechnungen von Gandjour et al. (2011) auf 44.736 Euro pro qualitätsbezogenem Lebensjahr beziehungsweise insgesamt zwischen 173 Millionen und 1,7 Milliarden Euro pro Jahr. Diese Daten beziehen sich auf ein 8-jähriges Follow-Up der MADIT-II-Daten und die Schätzung von etwa 190.000 Fällen von Myokardinfarkt in Deutschland pro Jahr, von denen circa ein Viertel eine stark eingeschränkte LVEF aufweist.

Aus den genannten Gründen ist es ausgesprochen wichtig, zu identifizieren, welche Patienten den größten Nutzen einer ICD-Implantation haben. Die Risikostratifizierung anhand klinischer Studien kann hierzu einen wichtigen Beitrag leisten. Oft haben diese Studien aber zahlreiche Ein- und Ausschlusskriterien und die untersuchten Populationen sind somit nicht direkt vergleichbar mit dem klinischen Alltag. Die hier vorliegende Arbeit untersucht eine umfassende Patientenpopulation, die über einen Zeitraum von 13 Jahren in einem tertiären Zentrum, nämlich der Universitätsmedizin Göt- 
tingen, einen ICD zur Primär- oder Sekundärprophylaxe des plötzlichen Herztodes erhalten hatte.

Die Analyse umfasst Prädiktoren für appropriate ICD-Schocks, was vor allem dazu dient, Patienten auszuwählen, die den größten Nutzen einer wirksamen antiarrhythmischen Therapie haben. Dies kann, soweit noch nicht erfolgt, eine ICDImplantation, andererseits eine zusätzliche antiarrhythmische Medikation sein.

Außerdem ist es wichtig, eine Implantation nur in Betracht zu ziehen, wenn die Patienten von einem ICD voraussichtlich auch profitieren. Daher wird in dieser Arbeit eine Analyse von klinischen Parametern durchgeführt, die Hinweise geben auf einen möglichen Tod ohne vorherige appropriate ICD-Schocks, also solche Patienten, die im Nachhinein keinen Nutzen der ICD-Therapie gehabt hätten.

Da vor allem inappropriate Schocks eine Belastung für Patienten darstellen, sollten auch hierfür Prädiktoren analysiert werden, um Strategien zur Prävention zu entwickeln oder Patienten mit generell höherem Risiko aufzuklären, um damit die Akzeptanz der potentiell lebensrettenden Therapie mittels ICD möglichst zu erhöhen. 


\section{Patienten und Methoden}

\subsection{Patientenkollektiv}

In die Auswertung eingeschlossen wurden 1484 konsekutive Patienten, denen ein ICD in der Universitätsmedizin Göttingen im Zeitraum von Januar 1998 bis Oktober 2010 implantiert wurde.

Die Patienten waren im Mittel $64 \pm 13$ Jahre alt, bei der Geschlechtsverteilung überwog der männliche Anteil deutlich (1200 Männer, 81\%).

\subsection{ICD-Implantation}

Die Implantation erfolgte zu 54\% aus primärprophylaktischer Indikation und bei $46 \%$ der Patienten zur Sekundärprophylaxe nach malignen ventrikulären Arrhythmien.

Eine umfassende kardiologische Diagnostik, unter anderem zur Evaluation der linksventrikulären Funktion ging der ICD-Implantation voraus. Nach Hautschnitt in der klavikulären Region wurde entweder die Vena cephalica oder die Vena subclavia punktiert, die Sonden transvenös vorgeschoben und im Myokard verschraubt. Alle ICD-Geräte wurden pektoral implantiert, entweder in eine subkutane oder eine submuskuläre Aggregattasche.

Da bei linksthorakaler Implantation ein physikalisch besseres Defibrillationsfeld vorliegt (Aggregatgehäuse als Anode), wurde die überwiegende Mehrheit der ICD linksthorakal implantiert (94\%). Gründe für Abweichungen waren z.B. eine Venenthrombose links oder ein Wechsel von einem Schrittmacher zu einem ICD (SystemUpgrade) in eine bereits vorhandene Aggregattasche rechts.

Einkammer-ICD haben eine einzelne Sonde, die meist im apikalen Myokard des rechten Ventrikels verschraubt wird und sowohl zur Abgabe von Schocks als auch zur antibradykarden Stimulation genutzt werden kann.

Das Vorliegen einer Schrittmacherindikation wie Sinusbradykardie oder anderer Sinusknotendysfunktionen beziehungsweise hochgradigen AV-Blockierungen ist eine Indikation für einen Zweikammer-ICD. Durch die zusätzlich im rechten Vorhof platzierte Sonde können Vorhof und Ventrikel sequenziell stimuliert und so eine hämodynamische Verbesserung erzielt werden. Ebenfalls ein Zweikammer-Gerät erhalten Patienten mit permanentem Vorhofflimmern. Zusätzliche atriale Detektionsalgorithmen können dabei zur besseren Unterscheidung von supraventrikulären und 
ventrikulären Tachyarrhythmien angewendet werden. Bei Vorliegen einer hochgradigen Herzinsuffizienz (NYHA-Stadium III / IV) trotz optimaler medikamentöser Therapie und eines kompletten Linksschenkelblocks (QRS-Breite $>120 \mathrm{~ms}$ ) beziehungsweise einer echokardiographisch gesicherten ventrikulären Asynchronizität hat sich eine kardiale Resynchronisationstherapie mittels eines CRT-D als vorteilhaft erwiesen. Dabei wird eine dritte Sonde in der Koronarsinus-Vene platziert und ermöglicht eine biventrikuläre Stimulation und Defibrillation. Nach Platzierung der Sonden und eventueller Relokation, bis sich zufriedenstellende Ergebnisse für die Stimulationsund Detektionswerte ergeben, erfolgt eine Testung der Defibrillationsschwelle (DFT, defibrillation treshold), das heißt der niedrigsten Energie, mit der eine erfolgreiche Defibrillation ausgelöst werden kann. Dazu wird induziertes Kammerflimmern mit jeweils sinkenden Energien terminiert, der niedrigste Wert muss bestätigt werden. Die postoperative Röntgenkontrolle dient der Beurteilung des regelrechten Sitzes des Aggregats und der Sonden als auch dem Ausschluss von Komplikationen, z.B. eines Pneumothorax nach Punktion der Vena sublavia.

Abbildung 1 zeigt ein konventionelles Röntgenbild in posterior-anteriorem Strahlengang. Zu sehen ist der links thorakal implantierte Kardioverter-Defibrillator vom Typ CRT-D (Boston Scientific Cognis $\left.\AA^{\circledR}\right)$.

In Abbildung 2 ist ein konventionelles Röntgenbild des gleichen Patienten in seitlichem Strahlengang dargestellt. Gut zu sehen ist die Projektion der drei Sonden auf den rechten Ventrikel, den rechten Vorhof und den Koronarsinus. 
Abbildung 1: Röntgenbild (posterior-anteriorer Strahlengang)

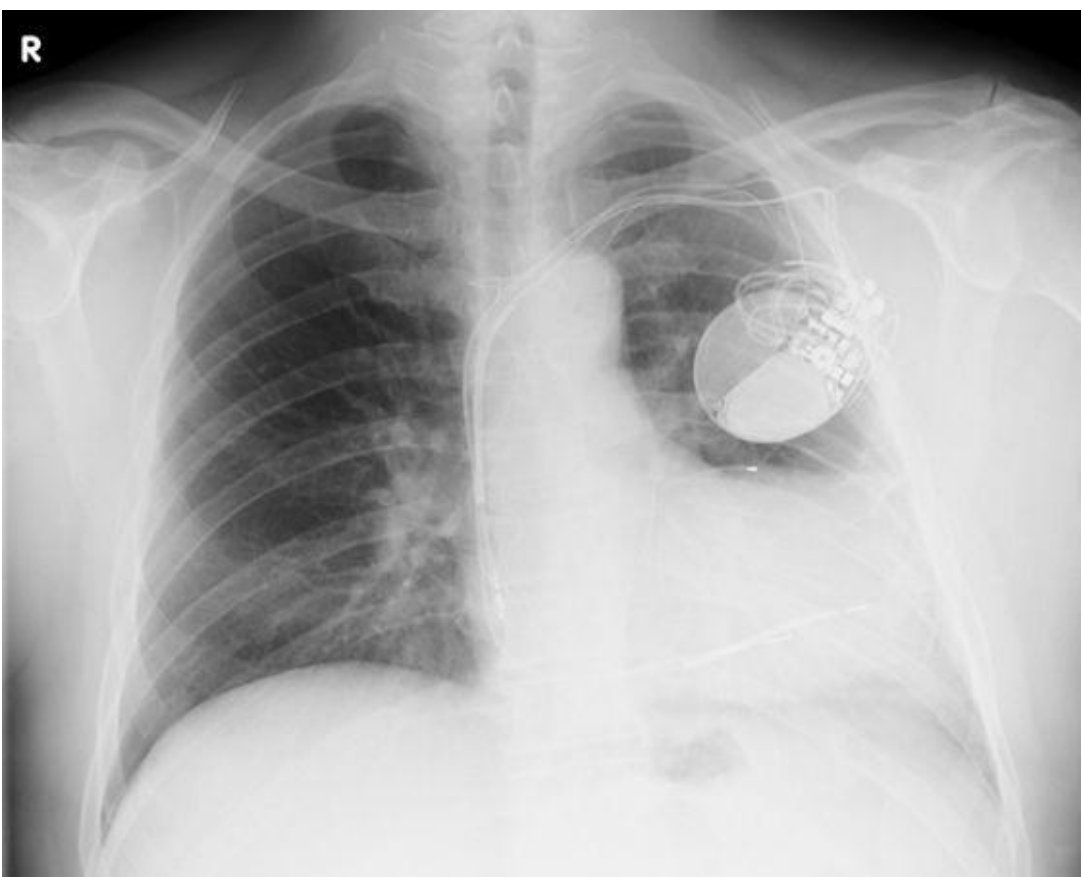

Abbildung 2: Röntgenbild (seitlicher Strahlengang)

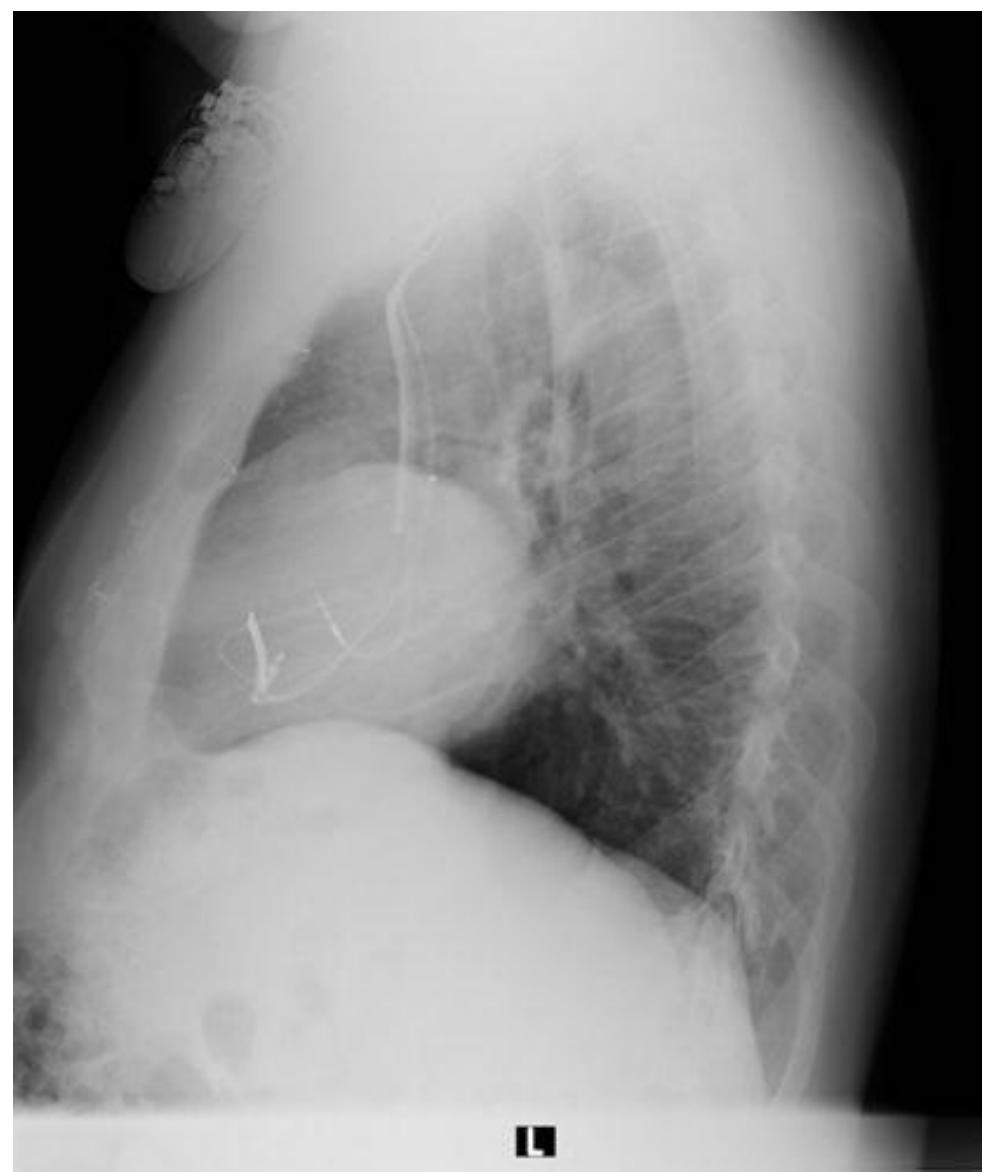




\subsection{ICD-Programmierung}

Die Programmierung der ICD-Geräte erfolgte in standardisierter Form, bei Notwendigkeit erfolgten patientenspezifische Anpassungen. Zur Therapie von eventuell auftretenden Bradykardien wurde bei allen Patienten eine antibradykarde Stimulation bei Frequenzen unter 40 pro Minute aktiviert. Die Untergrenze der VT-Zone wurde entweder mit 160-170 bpm (beats per minute, Schläge pro Minute) bei Patienten ohne spontane ventrikuläre Tachykardien programmiert, beziehungsweise mit 10 Schlägen unterhalb der Frequenz der niedrigsten aufgetretenen Tachykardie. Bei Einnahme von Amiodaron wurde die Grenze nochmals um 10 Schläge nach unten verschoben, da eine medikamentös bedingte Verlangsamung ventrikulärer Tachykardien auftreten kann. Als Detektionskriterien in der VT-Zone müssen 20 konsekutive Schläge den Kriterien entsprechen (NID, number of intervals detected 20/20), was bei einer kurzzeitig unterbrochenen Tachykardie zu einem erneuten Beginn der Analyse führt und die vorzeitige Therapie eventuell spontan terminierender Arrhythmien verhindern soll. Die Verzögerungszeit bis zur Therapieabgabe betrug 2,5 Sekunden. Als Therapie in der VT-Zone war standardmäßig antitachykardes Pacing aktiviert (3mal Burst-Stimulation gefolgt von 3-mal Ramp-Stimulation). Es erfolgt somit zunächst der Versuch, ventrikuläre Tachykardien durch schnelle Stimulation zu terminieren, bevor bei fehlendem Erfolg ein Schock abgegeben wird.

Bei Patienten mit dokumentierten langsamen VT mit einer unteren Grenze von circa 120 bpm, die hämodynamisch gut toleriert werden, wurde die Programmierung einer dritten Therapiezone erwogen. Da schnelle ventrikuläre Tachykardien (FVT, fast ventricular tachycardia) in ihrer Zykluslänge eventuell mit der VF-Zone überlappen können, wird in dieser Zone die ATP-Abgabe aktiviert. Nur bei ausbleibender Terminierung kommt es zum nachfolgenden Schock.

Hingegen kommt es in der VF-Zone (Frequenzen > $230 \mathrm{bpm}$ und unregelmäßige Kammerkomplexe) bei allen Patienten zu einer unmittelbaren Therapie mittels biphasischem Schock mit maximaler Energie (30-35 Joule je nach Aggregat). In der VF-Zone beträgt die NID 18/24 Schläge und die Detektionszeit 1 Sekunde.

Um vor allem inappropriaten Therapien vorzubeugen, gibt es zahlreiche Diskriminationsalgorithmen, die zur besseren Unterscheidung von ventrikulären und supraventrikulären Arrhythmien beitragen. Diese wurden regulär aktiviert, aufgrund der vielen 
unterschiedlichen Aggregattypen, die im Verlauf des untersuchten Zeitraums implantiert wurden, können hier aber nur Richtwerte angegeben werden.

Die Frequenzstabilität wird vor allem zur Detektion von Vorhofflimmern genutzt. Beträgt die Schwankung der R-R-Intervalle mehr als $30-50 \mathrm{~ms}$, ist von einer supraventrikulären Genese der Rhythmusstörung auszugehen und die Therapie wird zurückgehalten. Problematisch ist, dass Vorhofflimmern $>170$ bpm zunehmend regelmäßiger übergeleitet wird und auch ventrikuläre Tachykardien Zyklusschwankungen unterliegen können, vor allem unter antiarrhythmischer Medikation.

Ein weiteres Kriterium ist ein plötzlicher Frequenzsprung (sudden onset), der vor allem bei ventrikulären Tachykardien typisch ist. Dagegen kommt es bei Sinustachykardien zu einem allmählicheren Frequenzanstieg. Es werden die Mittelwerte der Intervalle mit den vorhergehenden Intervallen verglichen. Bei einer Verkürzung um $15-20 \%$ ist das Kriterium erfüllt und die Therapie wird eingeleitet. Bei ventrikulären Tachykardien, die unterhalb der VT-Zone beginnen und schneller werden, kann dieser Algorithmus die gewünschte Unterscheidung eventuell nicht leisten.

Da sich bei ventrikulären Tachyarrhythmien die QRS-Komplexe von denen bei Sinusrhythmus unterscheiden, kann die Analyse der Morphologie zur Differenzierung beitragen. Je nach Firma kommen verschiedene Mechanismen zum Einsatz, wie zum Beispiel Boston Rhythm ID ${ }^{\text {TM }}$ oder Medtronic Wavelet ${ }^{\mathrm{TM}}$. Gemeinsam ist innen der Vergleich von Kammerkomplexen mit einem zuvor gespeicherten Referenzkomplex bei Sinusrhythmus. Bei bestimmten, vorher definierten Abweichungen wird die Arrhythmie als ventrikulär angesehen und entsprechend therapiert.

\subsection{ICD-Therapie}

In Abbildung 3 ist der Ausdruck eines intrakardialen Elektrogramms (EGM) zu sehen, in dem eine spontane ventrikuläre Tachykardie mit einer durchschnittlichen Frequenz von $150 \mathrm{bpm}$ auftritt. Diese wird mittels antitachykardem Pacing (Ramp-Stimulation, Abnahme der Zykluslänge von Stimulus zu Stimulus) erfolgreich terminiert. Im Anschluss erfolgt eine regelrechte biventrikuläre Stimulation (Herzfrequenz 61 pro Minute). 
Abb. 3: ATP - Antitachykardes Pacing (EGM-Ausdruck)
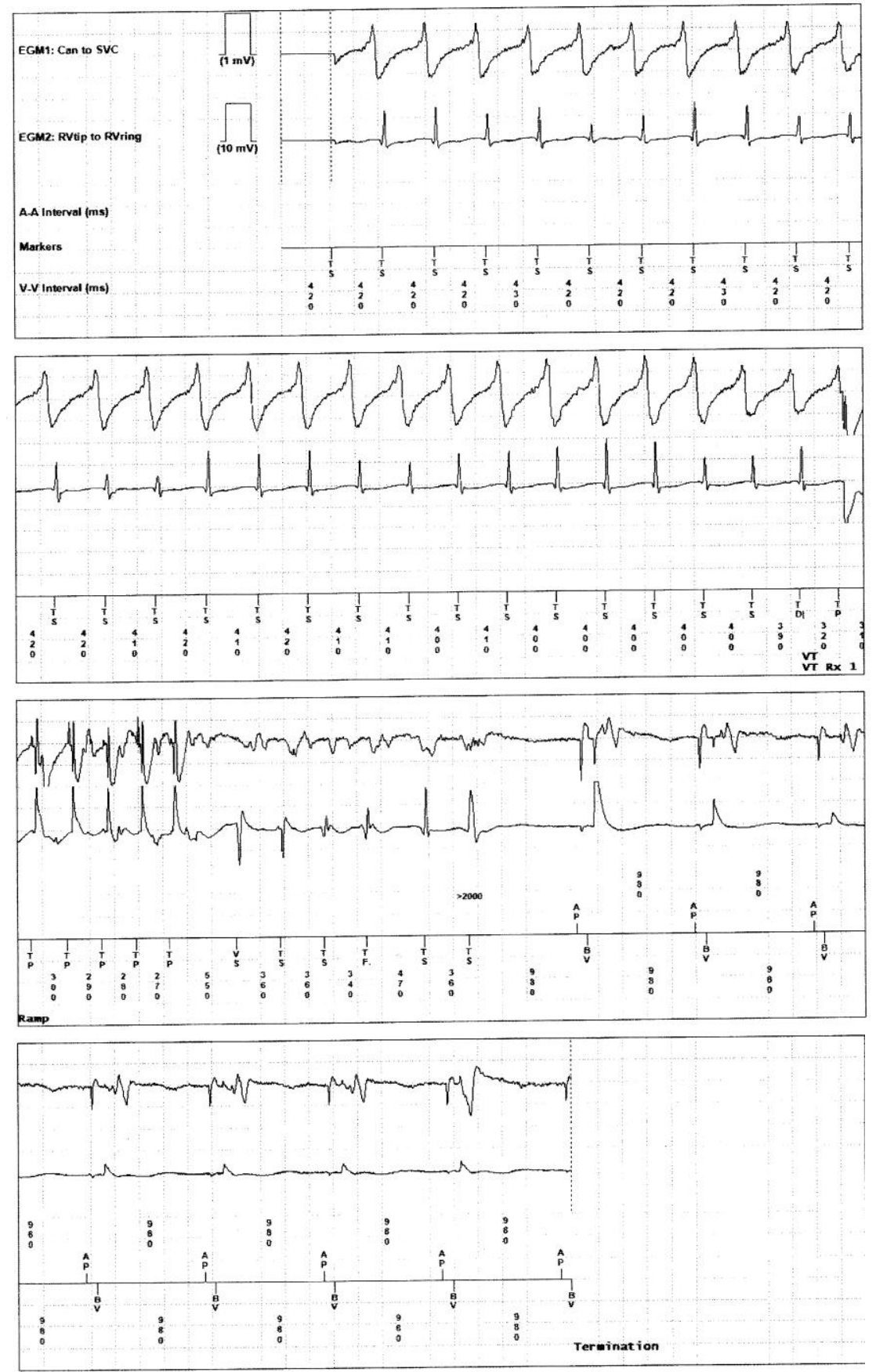

In der folgenden Abbildung 4 ist ein EGM dargestellt, in dem eine spontane ventrikuläre Tachykardie mit durchschnittlich 143 Schlägen pro Minute nach antitachykardem Pacing in Kammerflimmern übergeht und mittels eines ICD-Schocks mit 35 Joule terminiert wird. Im Anschluss zeigt sich eine regelrechte biventrikuläre Stimulation und eine Herzfrequenz von 80 bpm. 
Abb. 4: Appropriater Schock für VT (EGM-Ausdruck)

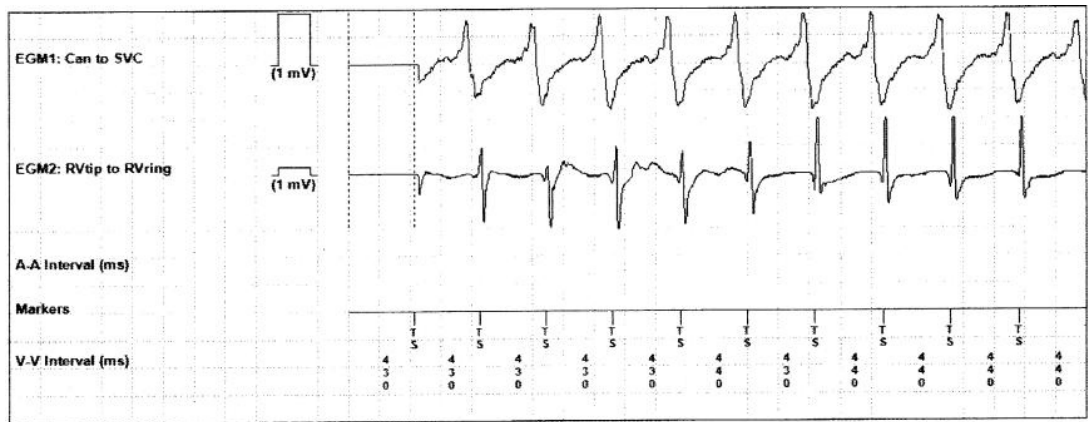

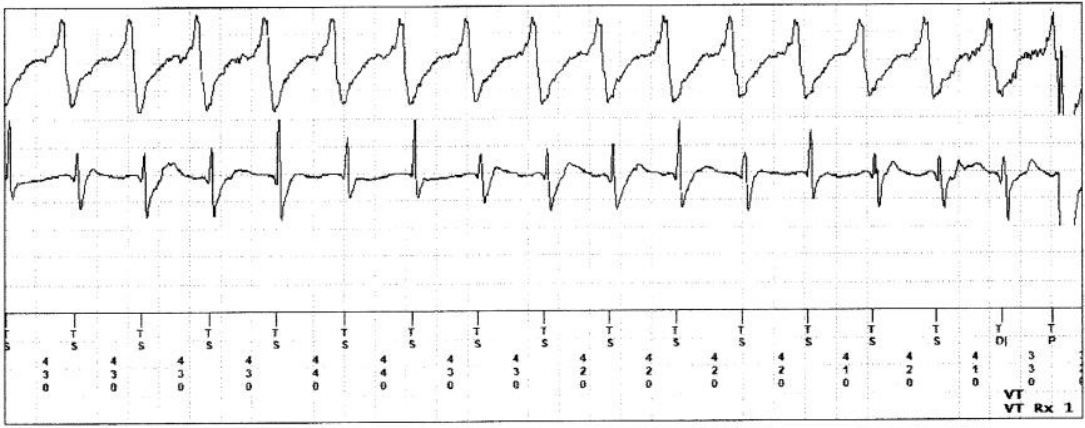

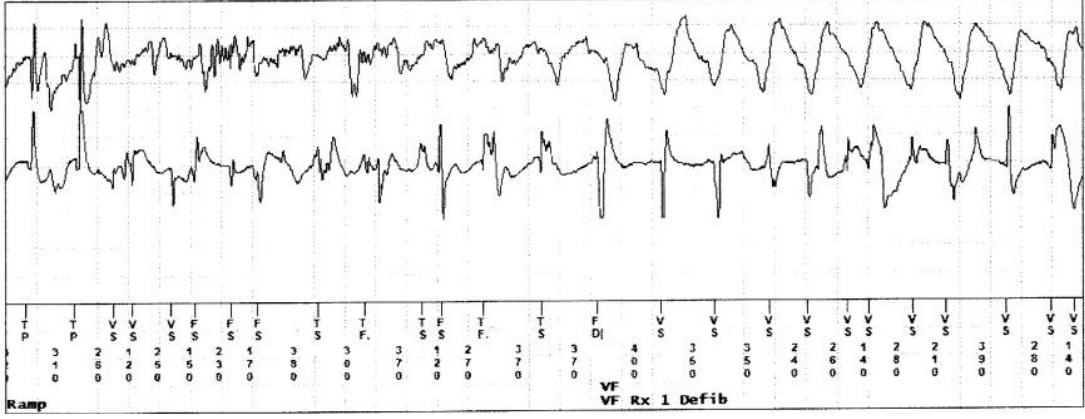

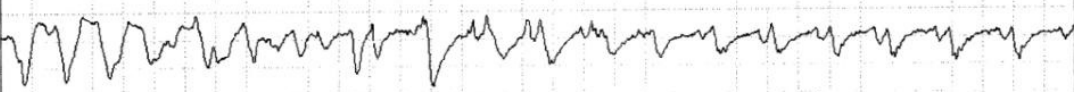

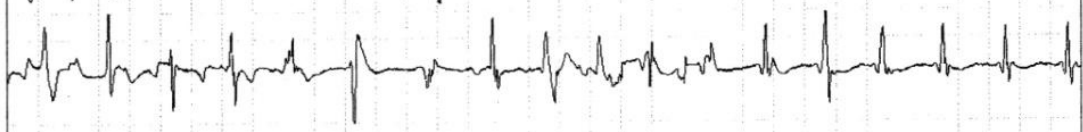

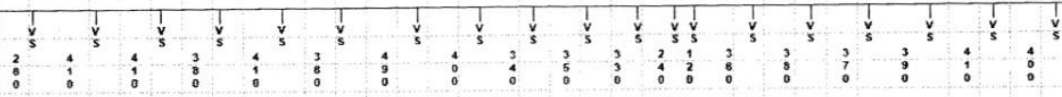

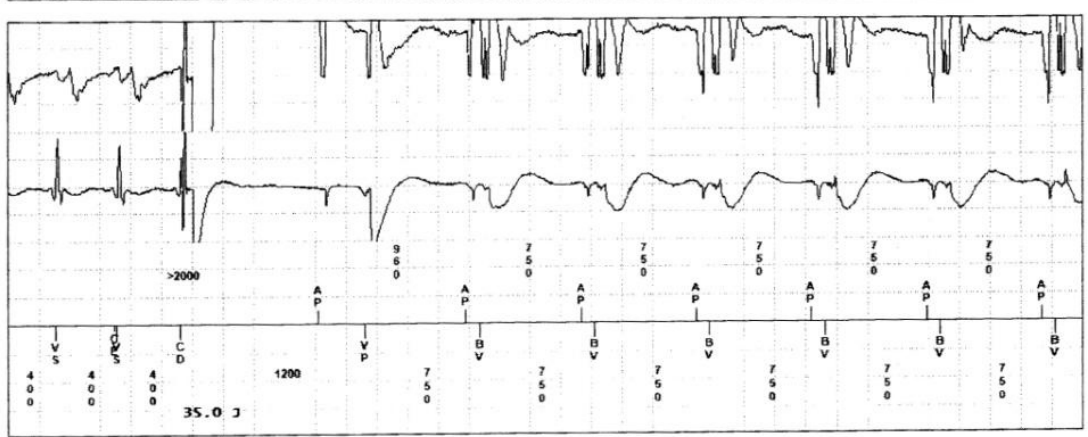




\subsection{Nachsorge}

Drei Monate nach Implantation wurden die Patienten erstmalig erneut einbestellt zur Kontrolle und Optimierung der Programmierungsparameter. Danach betrug das übliche Nachsorgeintervall sechs Monate. Bei Auftreten von Synkopen, abgegebenen Schocks, Verdacht auf Gerätekomplikationen oder einer klinischen Verschlechterung war eine Vorstellung in der Ambulanz jederzeit möglich.

Die routinemäßige Anamnese beinhaltete die aktuelle Medikation und eventuell bemerkte Rhythmusereignisse oder Synkopen, das Abfragen des Gerätes lieferte Angaben zu Batteriestatus und Therapieabgaben. Außerdem wurde die Stimulationsimpedanz, Defibrillationsimpedanz, R-Wellenamplitude und die Reizschwelle bestimmt, um Veränderungen zu erkennen, die z.B. auf eine Sondendislokation hindeuten. Zudem erfolgten regelmäßig eine körperliche Untersuchung mit Inspektion der Aggregattasche und das Prüfen von Herzinsuffizienzzeichen, außerdem die Aufzeichnungen der Vitalparameter und eine EKG-Analyse.

\subsection{Datenerhebung}

Die Datenerhebung erfolgte retrospektiv aus Implantationsprotokollen, Patientenakten und elektronischen Patientendaten. Die Nachbeobachtungszeit hinsichtlich ICDTherapien erfolgte aus Nachsorgeprotokollen der ICD-Ambulanz und wurde bis November 2012 durchgeführt. Die personenbezogenen Daten wurden in pseudonymisierter Form ausgewertet. Die Bewertung der ICD-Therapien anhand gespeicherter Elektrogramme hinsichtlich appropriater und inappropriater Therapien erfolgte in Zusammenarbeit mit einem Kardiologen.

Um Informationen zur Mortalität zu erhalten, wurden Auskünfte der jeweiligen Einwohnermeldeämter des letzten bekannten Wohnorts der Patienten eingeholt. Waren Patienten verzogen, so wurde beim nächsten zuständigen Einwohnermeldeamt nachgefragt. Nach fünf Umzügen in verschiedene Zuständigkeitsbereiche wurde die Nachverfolgung beendet. So konnten Mortalitätsdaten zu 1458 Patienten (98,2\%) erhoben werden.

Die Patienten, die bis Oktober 2011 keinen Schock dokumentiert hatten und nicht verstorben waren, wurden persönlich angeschrieben und bezüglich des Auftretens von Schocks befragt. Ein diesbezüglicher Ethikantrag wurde von der EthikKommission der Medizinischen Fakultät genehmigt (Antragsnummer 30/10/11). 
Ebenso wurden die behandelnden Hausärzte angeschrieben oder telefonisch kontaktiert sowie Daten über die Nachsorge von externen Krankenhäusern angefordert. Insgesamt konnten zusätzliche Daten zu 537 Patienten erhoben und die Ergebnisse vervollständigt werden.

\subsection{Statistische Methoden}

Alle Auswertungen erfolgten mit dem Programm IBM $₫$ SPSS $®$ Statistics Version 20 für Windows (SPSS GmbH Software, München).

Die Angabe von metrischen Daten erfolgte als Mittelwert \pm Standardabweichung, die Darstellung nominaler Daten als absolute und relative Häufigkeiten. Dabei wurden die Ergebnisse auf eine Stelle nach dem Komma gerundet. Mit dem exakten Test nach Fisher wurden kategoriale Variablen auf Unterschiede in den Verteilungshäufigkeiten getestet.

Zur Untersuchung von Prädiktoren für das Auftreten von appropriaten und inappropriaten ICD-Therapien beziehungsweise von Todesfällen, bevor es zu einer appropriaten Therapie kam, wurden jeweils Cox-Regressionsanalysen durchgeführt. Dazu wurden zunächst 23 klinisch relevante Variablen ausgewählt und in einer univariaten Analyse jeweils einzeln auf einen möglichen Einfluss hin untersucht. Das Signifikanzniveau in diesem ersten Schritt wurde auf $p \leq 0,10$ festgelegt. Im zweiten Schritt wurden die als statistisch signifikant angesehenen Variablen der univariaten Analyse in ein multivariates Modell übernommen und in schrittweiser Rückwärts-Selektion (nach der Wald-Teststatistik) ausgewertet. Das heißt, dass Schritt für Schritt diejenigen Variablen aus dem Modell entfernt wurden, die bei jeweiliger Interaktion einen $p$ Wert $\geq 0,05$ aufwiesen.

Für die endgültig zum multivariaten Regressionsmodell beitragenden Parameter wurden jeweils Kaplan-Meier-Überlebenskurven zum Vergleich der Patientenpopulationen angefertigt und mittels des Log-Rank-Tests verglichen.

Bis auf die Ausnahme der univariaten Cox-Regressionsanalysen wurden Ergebnisse ab einer Irrtumswahrscheinlichkeit von $p \leq 0,05$ als statistisch signifikant angesehen. 


\section{Ergebnisse}

\subsection{Charakterisierung des Patientenkollektivs}

Aufgrund der retrospektiven Erhebung ergaben sich unterschiedlich vollständige Datensätze. Daher unterscheidet sich die jeweilige Anzahl der betrachteten Patienten (Basis-n) je nach thematischem Schwerpunkt. Die Angaben beziehen sich jeweils nur auf die Erstimplantation des ICD.

Von den 1484 Patienten, die im Folgenden beschrieben werden, waren 1200 Patienten männlich (81\%) und 284 weiblich (19\%). Bei Erstimplantation waren die Patienten im Durchschnitt $64 \pm 13$ Jahre alt. Die gruppierte Altersverteilung ist in Abbildung 5 dargestellt.

Abbildung 5: Alter bei Erstimplantation des ICD

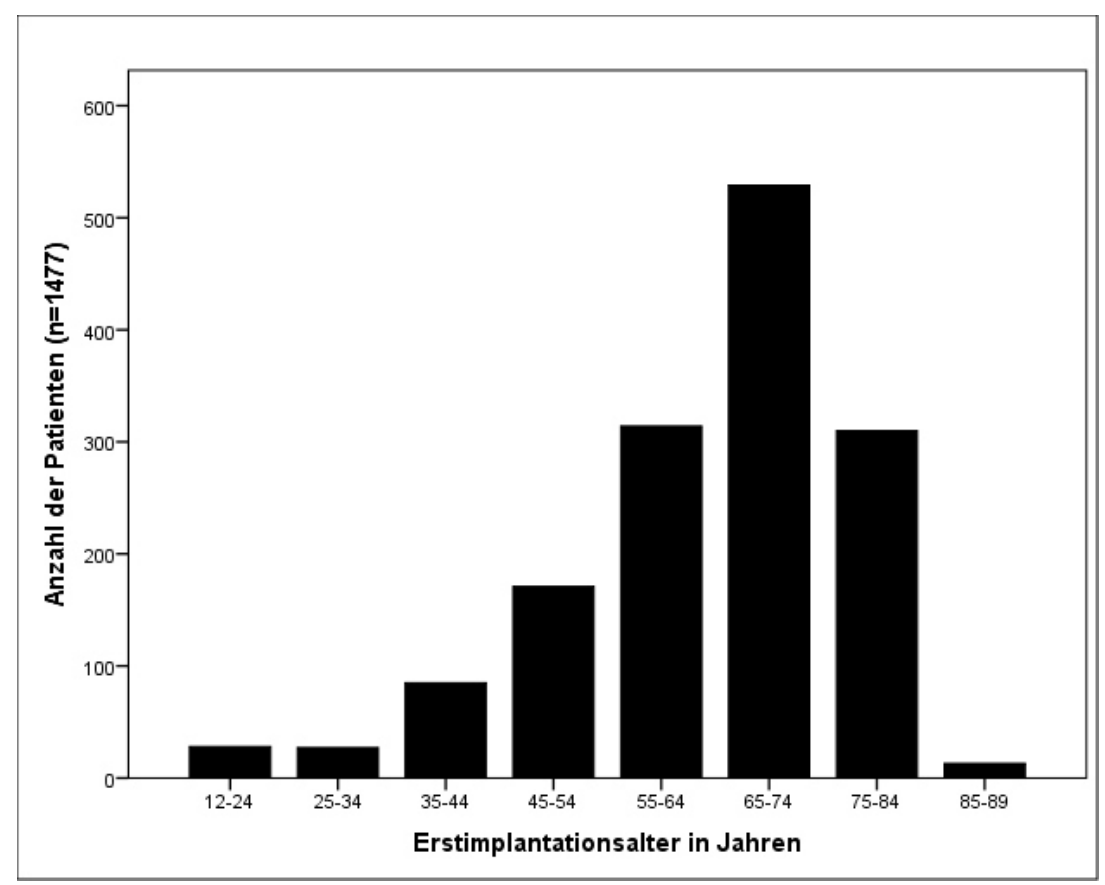


Tabelle 1 zeigt die Häufigkeiten der kardialen Grunderkrankungen in der untersuchten Population. Diese können einzeln oder auch kombiniert vorgelegen haben.

Tabelle 1: Kardiale Grunderkrankung ( $n=1421)$

\begin{tabular}{lrr}
\hline & absolut & in \% \\
\hline keine Grunderkrankung & 81 & 5,7 \\
KHK - 1 Gefäß & 409 & 28,8 \\
KHK - 2 Gefäße & 184 & 12,9 \\
KHK - 3 Gefäße & 388 & 27,3 \\
Z.n. Myokardinfarkt & 603 & 42,4 \\
Mitralinsuffizienz > II & 311 & 21,9 \\
kongenitale Herzvitien & 20 & 1,4 \\
dilatative Kardiomyopathie & 494 & 34,8 \\
hypertrophe Kardiomyopathie & 38 & 2,7 \\
Long-QT-Syndrom & 15 & 1,1 \\
Brugada-Syndrom & 6 & 0,4 \\
ARVC & 6 & 0,4 \\
\hline
\end{tabular}

KHK: koronare Herzkrankheit

Z.n.: Zustand nach

ARVC: arrhythmogene rechtsventrikuläre Kardiomyopathie

Der Grundrhythmus bei Erstimplantation ist in Tabelle 2 aufgeführt. Es wurde nicht zwischen intermittierendem und persistierendem Vorhofflimmern unterschieden.

Tabelle 2: Grundrhythmus bei Erstimplantation ( $n=1395)$

\begin{tabular}{lrc} 
& absolut & in \% \\
\hline Sinusrhythmus & 831 & 59,6 \\
Vorhofflimmern & 564 & 40,4 \\
\hline
\end{tabular}

Die durchschnittliche linksventrikuläre Ejektionsfraktion vor ICD-Implantation lag bei $30 \pm 12 \%$. Bei 88 Patienten (6\%) zeigte sich eine Hypertrophie des linken Ventrikels von mehr als $12 \mathrm{~mm}$. Die Unterteilung der LVEF in die Gruppen normal (>55\%), leichtgradig eingeschränkt (41-55\%), mittelgradig eingeschränkt (31-40\%) und schwer eingeschränkt $(\leq 30 \%)$ ist in Abbildung 6 dargestellt. 
Abbildung 6: Linksventrikuläre Ejektionsfraktion (LVEF)

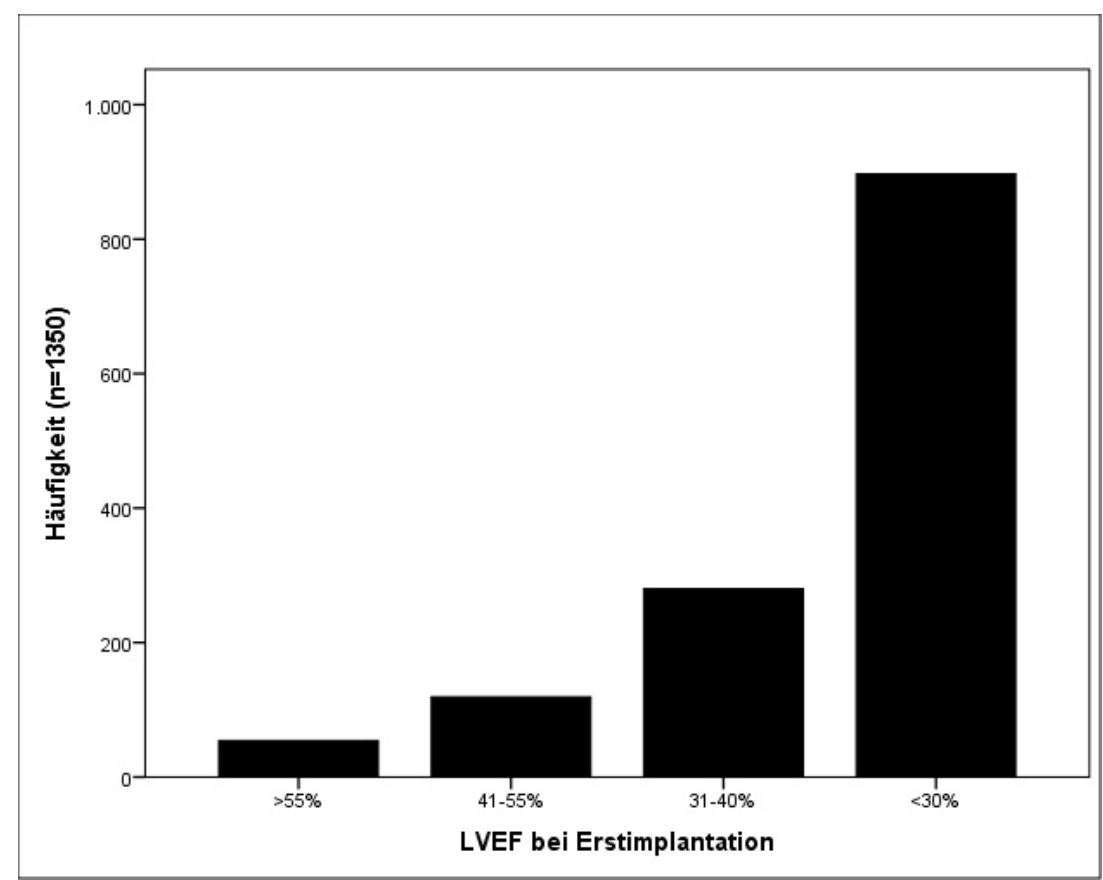

Eine Übersicht über die Art der Funktionsbestimmung der linksventrikulären Ejektionsfraktion gibt Tabelle 3 .

Tabelle 3: Funktionsbestimmung der LVEF ( $n=1350)$

\begin{tabular}{lrr}
\hline & absolut & in \% \\
\hline Angiographie des linken Ventrikels & 152 & 11,2 \\
Echokardiographie & 980 & 72,6 \\
andere (z.B. Magnetresonanztomographie) & 21 & 1,6 \\
Unbekannt & 197 & 14,6 \\
\hline
\end{tabular}

In Tabelle 4 sind die kardiologisch relevanten operativen Interventionen vor ICDImplantation dargestellt, die einzeln oder kombiniert vorgelegen haben können.

Tabelle 4: (Operative) Interventionen ( $n=1337)$

\begin{tabular}{lrr}
\hline & absolut & in \% \\
\hline Z.n. PCI & 397 & 29,7 \\
Z.n. ACVB & 365 & 27,3 \\
Z.n. Herzklappen-Operation & 106 & 7,9 \\
Z.n. Katheterablation & 40 & 3,0 \\
\hline Z.n.: Zustand nach & & \\
PCl: perkutane Koronarintervention & & \\
ACVB: aorto-coronarer Venen-Bypass & &
\end{tabular}


Weitere relevante nicht kardiale Erkrankungen der Patienten sind in Tabelle 5 aufgeführt. Als Kriterium für eine Depression galt eine medikamentöse antidepressive Therapie.

Tabelle 5: Nicht kardiale Grunderkrankungen ( $n=1293)$

\begin{tabular}{lrr}
\hline & absolut & in \% \\
\hline Z.n. zerebralen Durchblutungsstörungen & 130 & 10,1 \\
Z.n. peripherer Embolie & 96 & 7,4 \\
COPD & 211 & 16,3 \\
Malignom & 120 & 9,3 \\
Depression & 53 & 4,1 \\
\hline
\end{tabular}

Z.n.: Zustand nach

COPD: chronisch obstruktive Lungenerkrankung

Wichtige kardiovaskuläre Risikofaktoren sind arterielle Hypertonie (systolischer Blutdruck $\geq 140 \mathrm{~mm} \mathrm{Hg}$ und / oder diastolischer Blutdruck $\geq 90 \mathrm{~mm} \mathrm{Hg}$ ), Diabetes mellitus und Hyperlipoproteinämie. Die Häufigkeiten dieser Risikofaktoren in der Patientenpopulation werden in Tabelle 6 gezeigt, dabei sind Mehrfachnennungen möglich.

Nikotinkonsum stellt einen weiteren wichtigen kardiovaskulären Risikofaktor dar. Die Anzahl der Patienten mit aktuellem oder zurückliegendem Nikotinkonsum konnte aber aufgrund von unvollständiger Dokumentation nicht bestimmt werden.

Adipositas liegt ab einem Body-Mass-Index (BMI) von größer oder gleich $30 \mathrm{~kg} / \mathrm{m}^{2}$ vor und gilt als eigenständiger kardiovaskulärer Risikofaktor. Von den 827 Patienten mit vorliegenden Daten zu Größe und Gewicht waren nach diesen Kriterien 25\% adipös, der durchschnittliche BMI zum Zeitpunkt der Implantation betrug $28 \pm 8$ kg/m².

Tabelle 6: Kardiovaskuläre Risikofaktoren $(n=1244)$

\begin{tabular}{lrr}
\hline & absolut & in \% \\
\hline arterielle Hypertonie & 1046 & 84,1 \\
Typ-1-Diabetes mellitus & 152 & 12,2 \\
Typ-2-Diabetes mellitus & 199 & 16,0 \\
Hyperlipoproteinämie & 634 & 51,0 \\
\hline
\end{tabular}

Bei 84\% (912/1092) der Patienten, über die entsprechende Daten vorlagen, zeigten sich Zeichen der Herzinsuffizienz, vor allem Dyspnoe als Hinweis auf eine Linksherzinsuffizienz. Bei 180 Patienten gab es keine Beschwerden im Sinne einer Dyspnoe. 
Eine Darstellung entsprechend der Herzinsuffizienz-Stadien nach NYHA (New York Heart Association) findet sich in Abbildung 7.

Abbildung 7: Grad der Herzinsuffizienz (NYHA-Klassifikation)

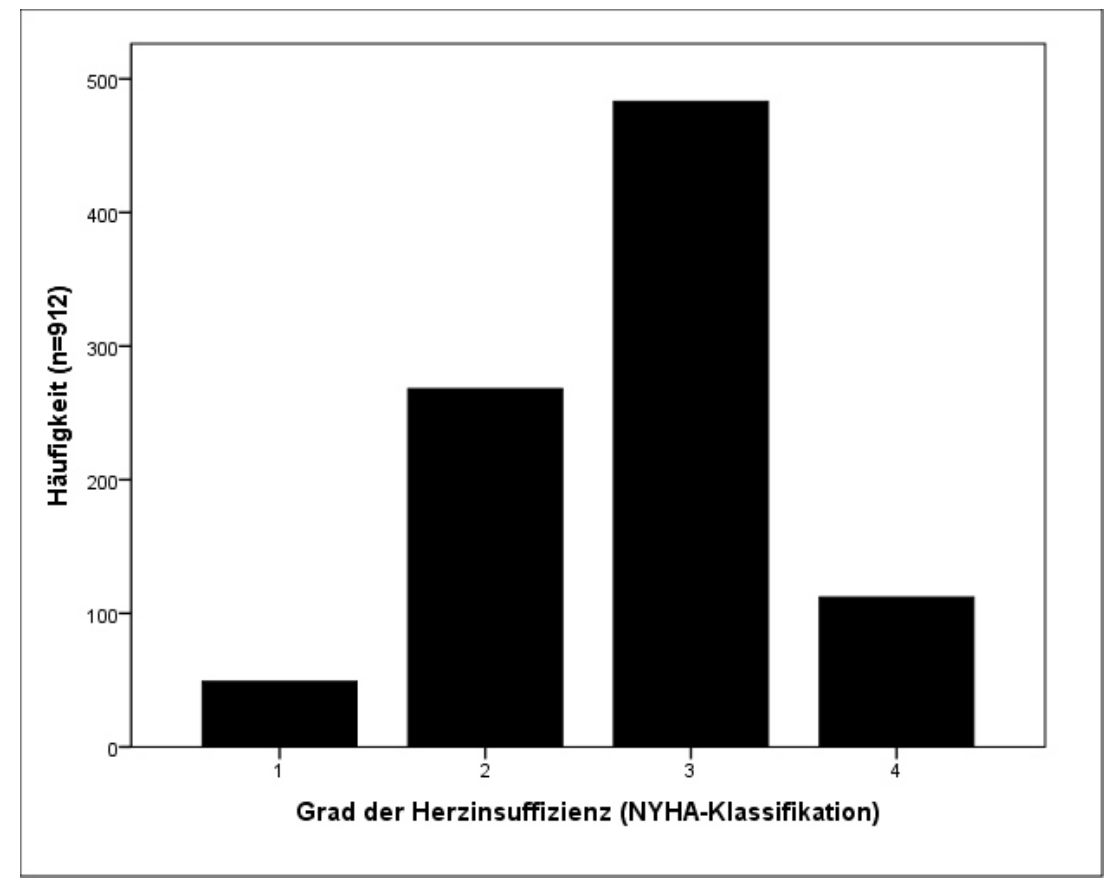

Die überwiegende Mehrheit der Patienten erhielt als Standardmedikation bei Herzinsuffizienz eine Therapie mit ACE-Hemmern beziehungsweise $\mathrm{AT}_{1}$-Antagonisten, Betablockern und Diuretika. Bei Bedarf kam eine Medikation mit Digitalisglykosiden, Aldosteronantagonisten und Statinen hinzu. Etwa 20\% der Patienten erhielten Amiodaron, andere Antiarrhythmika wurden nur selten eingesetzt.

Einen Überblick über die zum Zeitpunkt der ICD-Implantation bestehende Medikation liefert Tabelle 7. Hierbei kann es sich entweder um eine Mono- oder Kombinationstherapie handeln. 
Tabelle 7: Medikation bei ICD-Implantation ( $n=1263)$

\begin{tabular}{lrr}
\hline & absolut & in \% \\
\hline ACE-Hemmer / AT ${ }_{1}$-Antagonisten & 1097 & 86,9 \\
Betablocker & 1119 & 88,6 \\
Diuretika & 929 & 73,6 \\
Digitalisglykoside & 395 & 31,3 \\
Aldosteronantagonisten & 568 & 45,0 \\
Calciumkanalblocker & 115 & 9,1 \\
ASS & 765 & 60,6 \\
Clopidogrel & 255 & 20,2 \\
Cumarinderivate & 362 & 28,7 \\
Statine & 733 & 58,0 \\
Klasse-I-Antiarrhythmika & 16 & 1,3 \\
Sotalol & 10 & 0,8 \\
Amiodaron & 262 & 20,7 \\
Ivabradin & 5 & 0,4 \\
\hline ACT:Angor
\end{tabular}

ACE: angiotensin converting enzyme

$\mathrm{AT}_{1}$ : Angiotensin-II-Rezeptor-Subtyp-1

Präsynkopen sind gekennzeichnet durch Benommenheit, Seh- und Hörstörungen und Schweißausbrüche und können zu einer Synkope mit Bewusstseinsverlust führen. Diese klinischen Zeichen können Hinweise auf Herzrhythmusstörungen und nachfolgende zerebrale Minderperfusion liefern. Bei hochgradigen Rhythmusstörungen wie ventrikulären Tachykardien und Kammerflimmern können ein lebensbedrohlicher funktioneller Herzstillstand und eine Reanimationspflicht die Folge sein. Die Häufigkeit dieser klinischen Parameter im Vorfeld der ICD-Implantation zeigt die folgende Tabelle 8.

Tabelle 8: Klinische Präsentation ( $n=1293)$

\begin{tabular}{lrr}
\hline & absolut & in \% \\
\hline Z.n. Präsynkope & 66 & 5,1 \\
Z.n. Synkope & 151 & 11,7 \\
Z.n. Reanimation & 301 & 23,3 \\
\hline Z.n.: Zustand nach & &
\end{tabular}


Die primärprophylaktische Implantationsindikation war im untersuchten Patientenkollektiv etwas häufiger als die Sekundärprophylaxe des plötzlichen Herztodes. Bei 13 Patienten war eine Induzierbarkeit von ventrikulären Tachyarrhythmien in einer elektrophysiologischen Untersuchung alleinige Implantationsindikation. Tabelle 9 zeigt die jeweiligen Indikationen zur ICD-Implantation.

Tabelle 9: ICD-Implantationsindikation $(n=1450)$

\begin{tabular}{lrr}
\hline & absolut & in \% \\
\hline Primärprophylaxe & 781 & 53,9 \\
ventrikuläre Tachykardie (VT) & 339 & 23,4 \\
Kammerflimmern (VF) & 317 & 21,8 \\
induzierbare VT/VF & 13 & 0,9 \\
\hline
\end{tabular}

Die Verteilung der jeweiligen ICD-Gerätetypen wird in Tabelle 10 dargestellt. Je nach kardialer Grunderkrankung erhielten die Patienten einen Einkammer-, Zweikammeroder Dreikammer-ICD entsprechend den jeweils gültigen Leitlinien.

Tabelle 10: Gerätetyp bei Erstimplantation ( $n=1468)$

\begin{tabular}{lrr}
\hline & absolut & in \% \\
\hline Einkammer-ICD & 528 & 36,0 \\
Zweikammer-ICD & 486 & 33,1 \\
Dreikammer-ICD & 454 & 30,9 \\
\hline
\end{tabular}

Es wurden ICD von insgesamt drei Herstellerfirmen implantiert. Am häufigsten waren dies Geräte der Firma Medtronic (Medtronic Inc., Fridley, Minnesota, USA), gefolgt von Guidant beziehungsweise Boston Scientific (Guidant Corporation, seit 2006 Teil von Boston Scientific Corporation, Natick, Massachusetts, USA). Geräte der Firma Biotronik (Biotronik SE \& Co. KG, Berlin) wurden seit 2006 implantiert. Eine grafische Darstellung der Herstellerverteilung liefert Abbildung 8. 


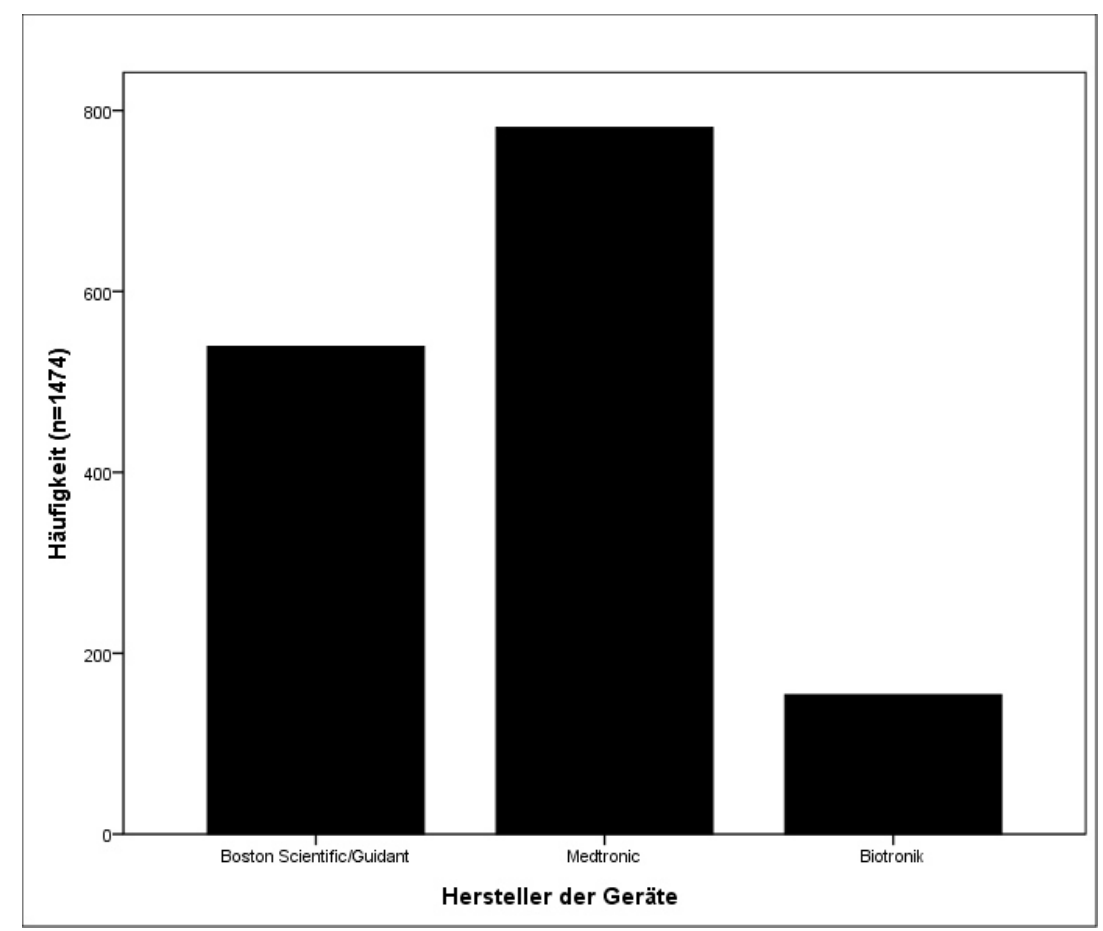

\subsection{ICD-Therapie}

Im Folgenden wird auf die abgegebenen Therapien der implantierbaren KardioverterDefibrillatoren eingegangen. Bei 180 Patienten war es nicht möglich, Daten zu erheben, da nach der Implantation keine Nachsorge in unserer Klinik stattfand und keine Daten von externen Quellen zugänglich waren.

Tabelle 11 zeigt die Häufigkeiten verschiedener Therapien, wobei ,jede ICDTherapie" sowohl die Abgabe von Schocks als auch ATP umfasst. Hierbei wird nicht zwischen appropriater und inappropriater Therapie unterschieden.

Als VT-Sturm wurden drei oder mehr isoliert auftretende Episoden von ventrikulärem Flimmern beziehungsweise ventrikulären Tachykardien innerhalb eines Zeitraums von 24 Stunden betrachtet, entsprechend der Definition von Exner et al. (2001). Die Art der abgegeben ICD-Therapien wurde hierbei nicht differenziert.

Die Häufigkeiten beziehen sich jeweils auf die Anzahl der Patienten $(n=1304)$, nicht auf die Anzahl der Therapien. 
Tabelle 11: Allgemeine ICD-Therapie ( $n=1304)$

\begin{tabular}{lrr}
\hline & absolut & in \% \\
\hline jede ICD-Therapie & 538 & 41,3 \\
jeder Schock & 408 & 31,3 \\
appropriate ATP-Abgabe & 288 & 22,1 \\
inappropriate ATP-Abgabe & 49 & 3,8 \\
VT-Sturm mit ICD-Therapie & 17 & 1,3 \\
\hline ATP: antitachykardes Pacing & & \\
VT: ventrikuläre Tachykardie & &
\end{tabular}

Als appropriate Therapie wurde ein Schock, der aufgrund von ventrikulärer Tachykardie oder Kammerflimmern abgegeben wurde, gewertet.

Ein Viertel der Patienten erhielt im Laufe der Nachbeobachtung einen oder mehrere appropriate Schocks, in Tabelle 12 wird zudem noch nach der jeweiligen Anzahl der Schocks unterteilt.

Die appropriate Therapie war in der Gruppe der sekundärprophylaktisch Implantierten signifikant größer als bei Patienten mit primärprophylaktischer Implantationsindikation (196 versus $135, p \leq 0,001$ ).

Tabelle 12: Appropriate ICD-Therapie $(n=1304)$

\begin{tabular}{crr}
\hline & absolut & in \% \\
\hline appropriater Schock & 331 & 25,4 \\
Anzahl: 1 & 135 & 10,4 \\
Anzahl: $2-4$ & 90 & 6,9 \\
Anzahl: $>4$ & 106 & 8,1 \\
\hline
\end{tabular}

Jede ICD-Therapie, die nicht aufgrund von ventrikulären Tachykardien oder Kammerflimmern abgegeben wurde, wurde als inappropriat betrachtet. Analog zur appropriaten Therapie werden in Tabelle 13 die Häufigkeiten der inappropriaten Schocks nach Anzahl und Häufigkeit aufgeführt. Daraus geht hervor, dass über die Hälfte der Patienten mit inappropriaten Schocks keine appropriate ICD-Therapie erhalten hatte. 
Tabelle 13: Inappropriate ICD-Therapie $(n=1304)$

\begin{tabular}{crr}
\hline & absolut & in \% \\
\hline inappropriater Schock & 134 & 10,3 \\
Anzahl: 1 & 72 & 5,5 \\
Anzahl: $2-4$ & 28 & 2,1 \\
Anzahl: $>4$ & 34 & 2,6 \\
inappropriate, aber keine appropriaten Schocks & 74 & 5,7 \\
\hline
\end{tabular}

Die Ursachen der inappropriaten Schocks sind in Tabelle 14 dargestellt. Neben schnell übergeleitetem Vorhofflimmern waren es vor allem Sinustachykardien oder supraventrikuläre Tachyarrhythmien, die zu inappropriaten Schocks führten. In etwas mehr als einem Drittel der Fälle war die Ursache ein fehlerhaftes Sensing, z.B. durch Oversensing von diaphragmalen Myopotentialen, P- oder T-Wellen, doppelter Wahrnehmung von R-Zacken oder Artefakten bedingt durch Sondendefekte, vor allem Isolationsdefekte oder Elektrodenbrüche, z.B. bei Subclavian-Crush-Syndrom.

In Bezug auf den Aggregattyp und das Auftreten inappropriater ICD-Schocks zeigte sich kein Unterschied zwischen Ein- und Zweikammer-ICD (39\% versus 42\%, $p=$ $0,408)$ in der untersuchten Population von 134 Patienten. Dagegen war ein CRT-D mit signifikant weniger inappropriaten Therapien im Vergleich mit Einkammer-ICD $(19 \%$ versus $39 \%, p=0,022)$ und Zweikammer-ICD $(19 \%$ versus $42 \%, p=0,002)$ assoziiert.

Tabelle 14: Ursachen der inappropriaten ICD-Therapie $(n=134)$

\begin{tabular}{lrr}
\hline & absolut & in \% \\
\hline Vorhofflimmern & 39 & 29,1 \\
supraventrikuläre Tachykardie & 35 & 26,1 \\
fehlerhaftes Sensing & 47 & 35,1 \\
Unbekannt & 13 & 9,7 \\
\hline
\end{tabular}

\subsection{Ereignisfreies Überleben}

Die Zeit bis zum ersten ICD-Schock wird in Abbildung 9 als Kaplan-Meier-Diagramm dargestellt. Zensiert wurden alle Patienten, die verstorben waren, bevor ein Schock 
auftrat. Unterhalb der Abbildung sind die verbliebenen Fälle zu den jeweiligen Zeitpunkten der Nachbeobachtung dargestellt.

Abbildung 9: Auftreten des ersten ICD-Schocks

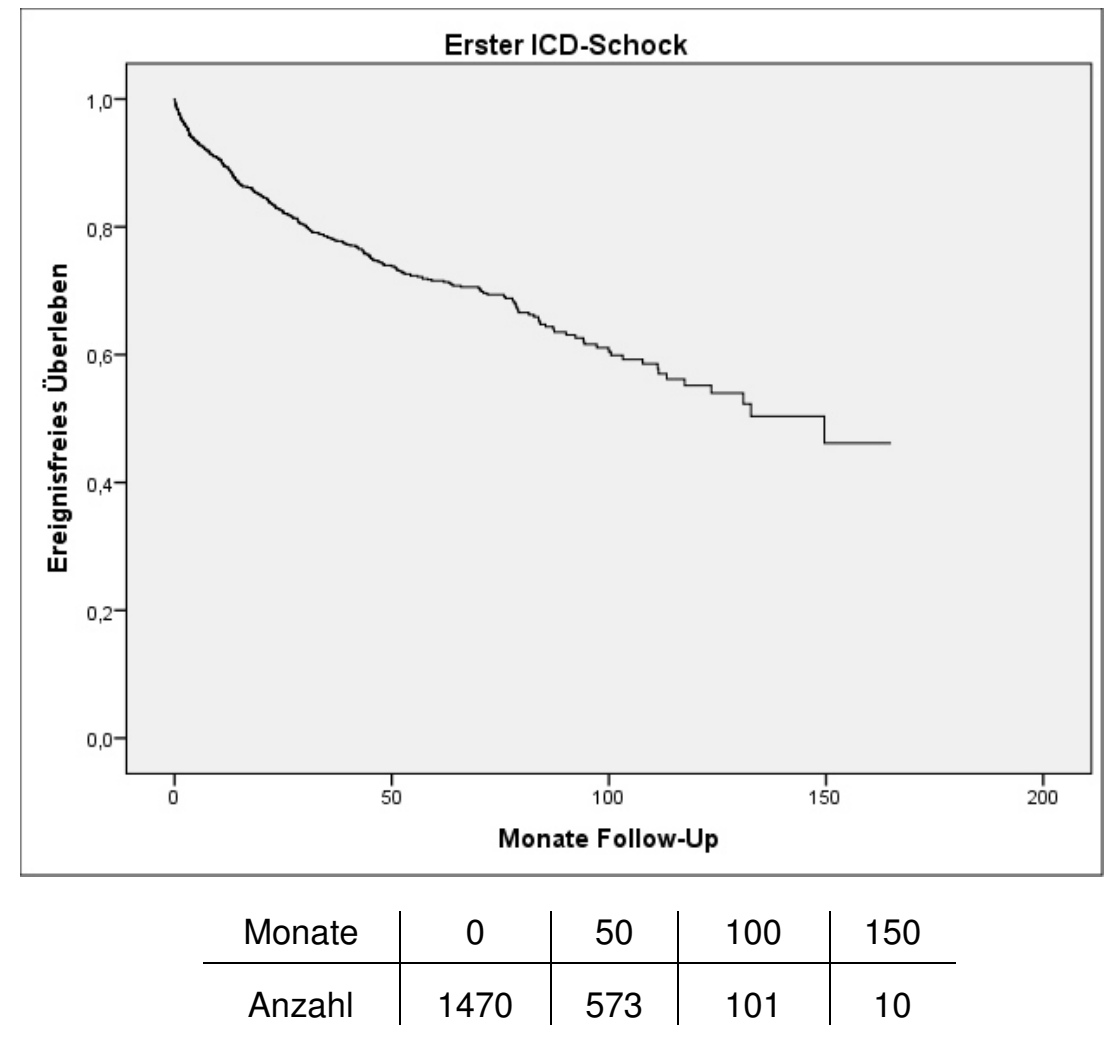

\subsection{Mortalität im Nachbeobachtungszeitraum}

Der Nachbeobachtungszeitraum endete im November 2012, das mittlere Follow-Up beträgt demnach $58 \pm 35$ Monate. Eine grafische Darstellung nach Kaplan-Meier der Mortalität in dem genannten Zeitraum zeigt Abbildung 10.

Bei 1458 Patienten (98\%) liegen Daten zur Mortalität vor. 481 Patienten (33\%) sind verstorben, davon $89(6 \%)$ im ersten Jahr nach Implantation. Das mittlere Todesalter lag bei $72 \pm 8$ Jahren. Die Mortalität unterschied sich nicht signifikant zwischen den Gruppen der Primär- und Sekundärprophylaxe (248 versus 233, $p=0,215$ ). 
Abbildung 10: Mortalität im Nachuntersuchungszeitraum

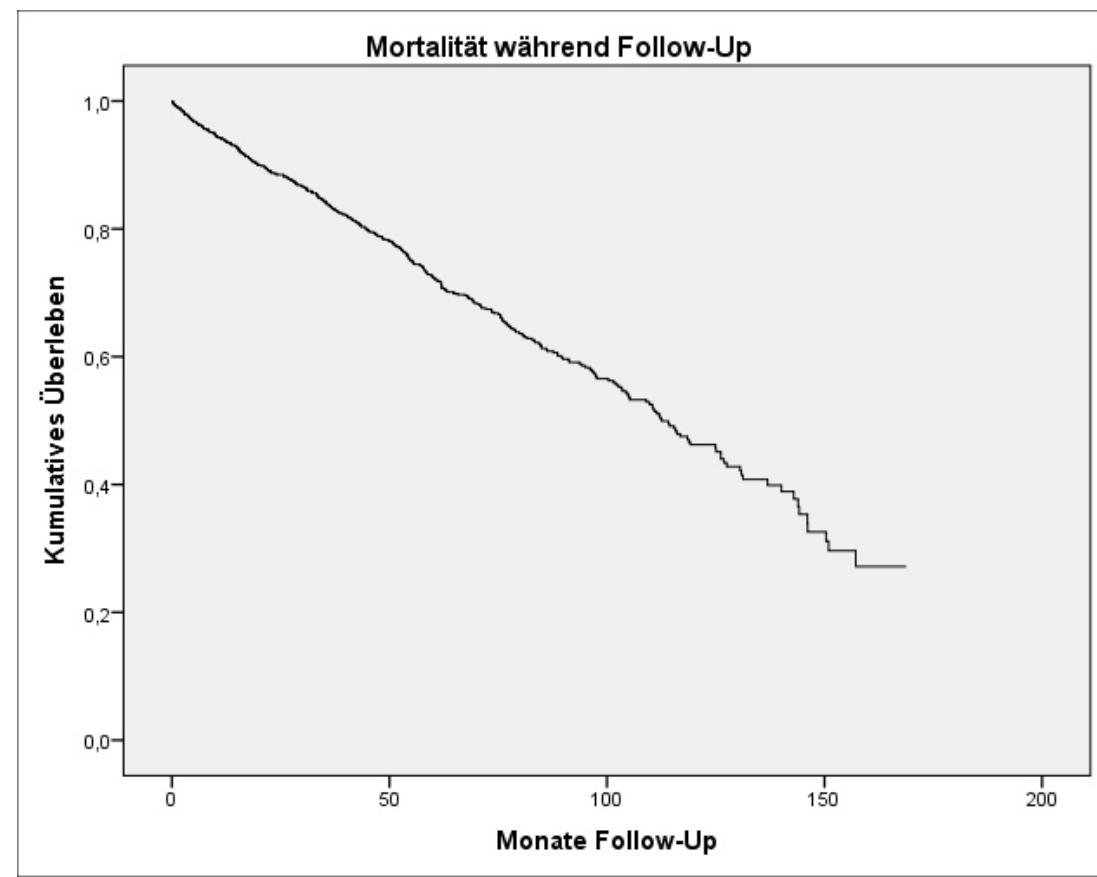

\begin{tabular}{c|c|c|c|c} 
Monate & 0 & 50 & 100 & 150 \\
\hline Anzahl & 1449 & 791 & 177 & 21
\end{tabular}

\subsection{Analyse von Prädiktoren für appropriate ICD-Schocks}

Um eine Aussage über eventuelle demographische oder klinische Parameter zur Vorhersage von Schocks zu treffen, wurden Cox-Regressionsanalysen durchgeführt. In Tabelle 15 sind die 23 Parameter aufgeführt, deren Einfluss auf das Ereignis „appropriater Schock“ untersucht wurde. Dabei wurden die Werte für das Implantationsalter und die linksventrikuläre Ejektionsfraktion nach dem Median in der Patientenpopulation geteilt.

NT-proBNP als laborchemischer Parameter für eine Herzinsuffizienz und Hinweis auf eine linksventrikuläre Dysfunktion wurde auf ein Vorliegen von pathologischen Werten (Norm < $400 \mathrm{pg} / \mathrm{ml}$ ) untersucht. Die QRS-Breite des 12-Kanal-EKG nach Implantation wurde ab einem Wert $>120 \mathrm{~ms}$ als Zeichen eines Schenkelblocks oder einer intraventrikulären Erregungsleitungsstörung in die Auswertung einbezogen.

\subsubsection{Univariate Cox-Regressionsanalyse}

Zuerst wurden die Parameter getrennt einer univariaten Regressionsanalyse unterzogen, um einen Einfluss auf das Auftreten von appropriaten Schocks zu untersu- 
chen. Die Ergebnisse sind in Tabelle 15 dargestellt, wobei die neun Parameter mit einem $p$-Wert $\leq 0,10$ hervorgehoben sind.

Tabelle 15: Univariate Cox-Regressionsanalyse für appropriate Schocks

\begin{tabular}{|c|c|c|c|}
\hline & p-Wert & Hazard Ratio & 95\% Konfidenzintervall \\
\hline Vorhofflimmern & 0,039 & 1,264 & $1,012-1,579$ \\
\hline Implantationsalter > $67 \mathrm{~J}$. & 0,674 & 0,954 & $0,764-1,191$ \\
\hline männliches Geschlecht & $<0,001$ & 1,891 & $1,353-2,642$ \\
\hline NYHA-Stadium III / IV & 0,210 & 0,852 & $0,662-1,095$ \\
\hline LVEF $<30 \%$ & 0,793 & 1,032 & $0,815-1,308$ \\
\hline Primärprophylaxe & $<0,001$ & 0,630 & $0,502-0,790$ \\
\hline KHK & 0,119 & 1,211 & $0,952-1,542$ \\
\hline DCM & 0,101 & 0,819 & $0,645-1,040$ \\
\hline Z.n. ACVB & 0,210 & 1,169 & $0,916-1,493$ \\
\hline Z.n. Myokardinfarkt & 0,068 & 1,229 & $0,985-1,533$ \\
\hline Diabetes mellitus & 0,926 & 0,988 & $0,764-1,278$ \\
\hline COPD & 0,075 & 1,314 & $0,973-1,775$ \\
\hline arterielle Hypertonie & 0,490 & 0,900 & $0,666-1,215$ \\
\hline Hyperlipoproteinämie & 0,789 & 1,032 & $0,819-1,301$ \\
\hline ACE-Hemmer & 0,305 & 0,846 & $0,615-1,164$ \\
\hline Betablocker & 0,184 & 0,793 & $0,563-1,117$ \\
\hline Statin & 0,023 & 0,764 & $0,606-0,964$ \\
\hline Diuretika & 0,646 & 1,063 & $0,819-1,380$ \\
\hline Sotalol & 0,094 & 2,131 & $0,880-5,162$ \\
\hline Amiodaron & $<0,001$ & 1,693 & $1,314-2,183$ \\
\hline Kreatinin-Wert erhöht & 0,078 & 1,167 & $0,983-1,384$ \\
\hline NT-proBNP > $400 \mathrm{pg} / \mathrm{ml}$ & 0,231 & 0,688 & $0,372-1,270$ \\
\hline QRS-Breite > $120 \mathrm{~ms}$ & 0,799 & 0,963 & $0,718-1,291$ \\
\hline
\end{tabular}

NYHA: New York Heart Association

LVEF: linksventrikuläre Ejektionsfraktion

KHK: koronare Herzkrankheit

DCM: dilatative Kardiomyopathie

Z.n.: Zustand nach

ACVB: aorto-coronarer Venen-Bypass

COPD: chronisch obstruktive Lungenerkrankung

ACE: angiotensin converting enzyme

BNP: brain natriuretic peptide

$\mathrm{pg} / \mathrm{ml}$ : Pikogramm je Milliliter

ms: Millisekunden 


\subsubsection{Multivariate Cox-Regressionsanalyse}

Insgesamt lagen 1144 vollständige Datensätze zur multivariaten Analyse vor. Davon war in 245 Fällen das Ereignis „appropriater Schock“ eingetreten.

Bei der schrittweisen Rückwärts-Selektion zeigten drei Parameter eine statistische Signifikanz mit einem p-Wert $\leq 0,05$ (siehe Tabelle 16).

Ein männliches Geschlecht und die Einnahme von Amiodaron vor Implantation waren mit einem erhöhten Risiko für appropriate Schocks assoziiert, eine primärprophylaktische Implantationsindikation mit einem niedrigeren Risiko.

Tabelle 16: Multivariates Cox-Regressionsmodell für appropriate Schocks

\begin{tabular}{lrrr}
\hline & p-Wert & Hazard Ratio & 95\% Konfidenzintervall \\
\hline männliches Geschlecht & 0,008 & 1,669 & $1,142-2,438$ \\
Primärprophylaxe & 0,014 & 0,719 & $0,553-0,934$ \\
Amiodaron & 0,002 & 1,554 & $1,171-2,063$ \\
\hline
\end{tabular}

\subsubsection{Kaplan-Meier-Überlebenskurven}

Der Einfluss der Parameter männliches Geschlecht, Primärprophylaxe und Einnahme von Amiodaron auf die Wahrscheinlichkeit des Auftretens von appropriaten ICDSchocks wurde jeweils in Kaplan-Meier-Überlebenskurven dargestellt (siehe Abbildungen 11 - 13). 
Abbildung 11: Appropriater Schock (Geschlecht)

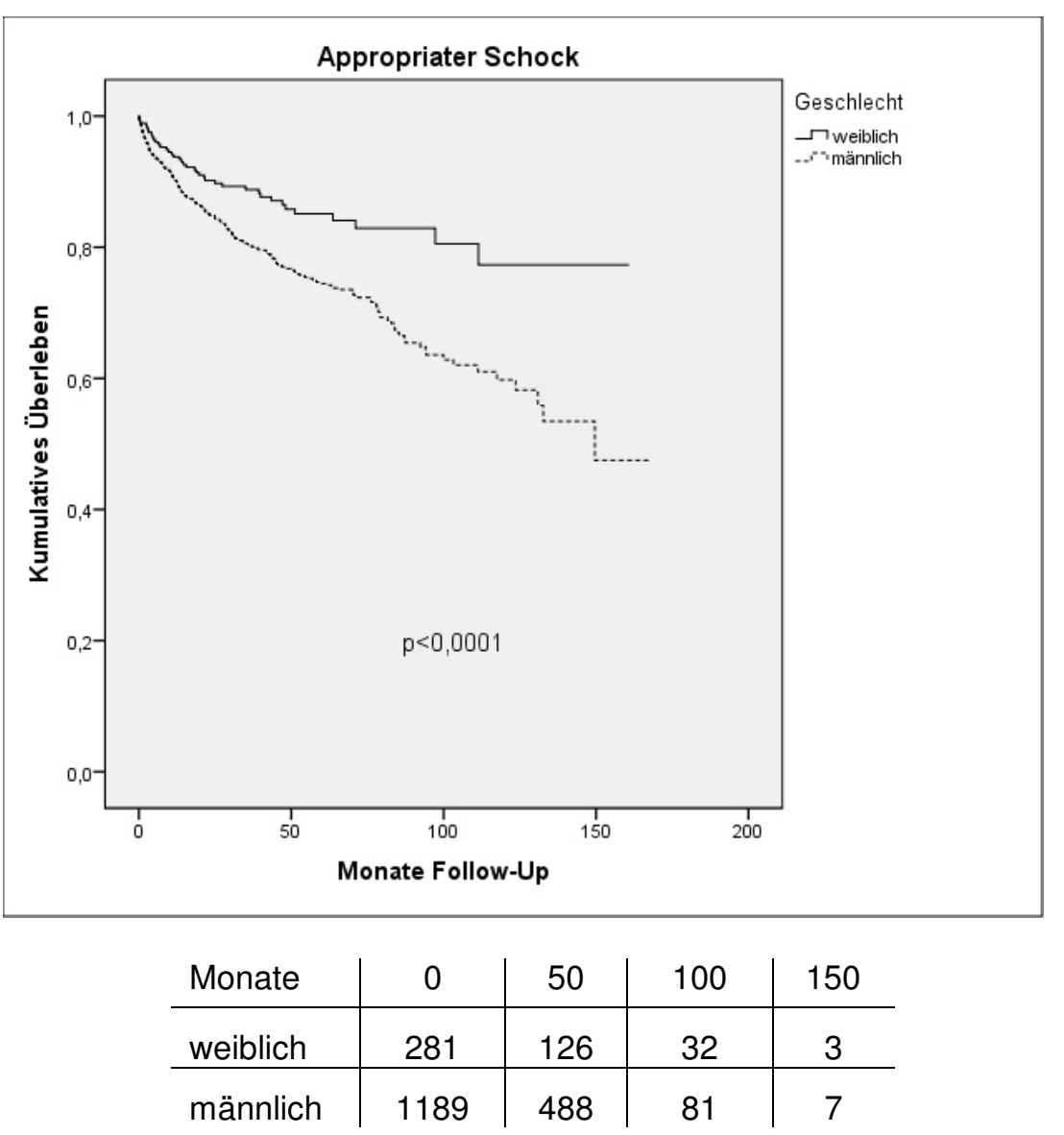

Abbildung 12: Appropriater Schock (Primärprophylaxe)

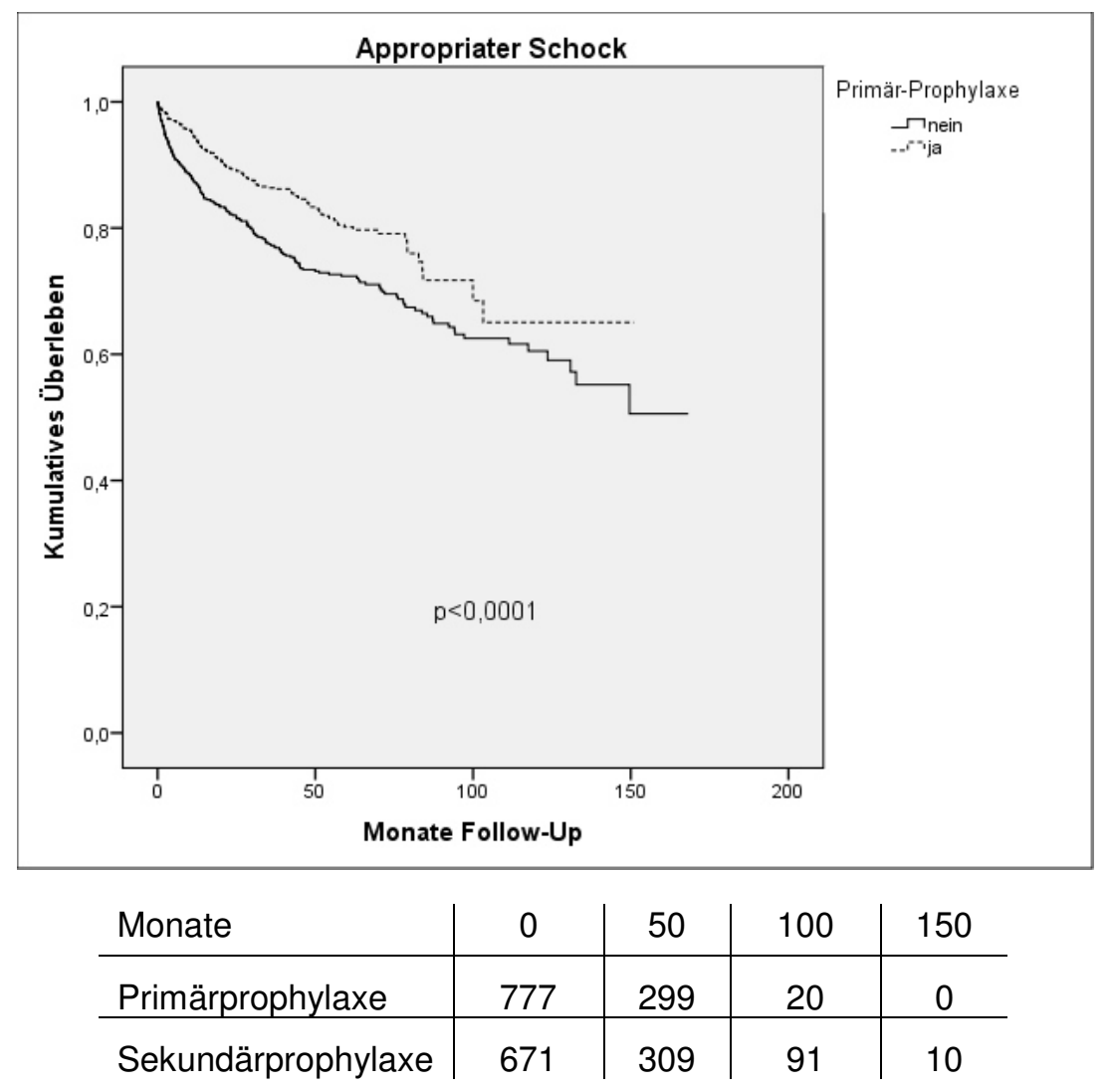


Abbildung 13: Appropriater Schock (Amiodaroneinnahme)

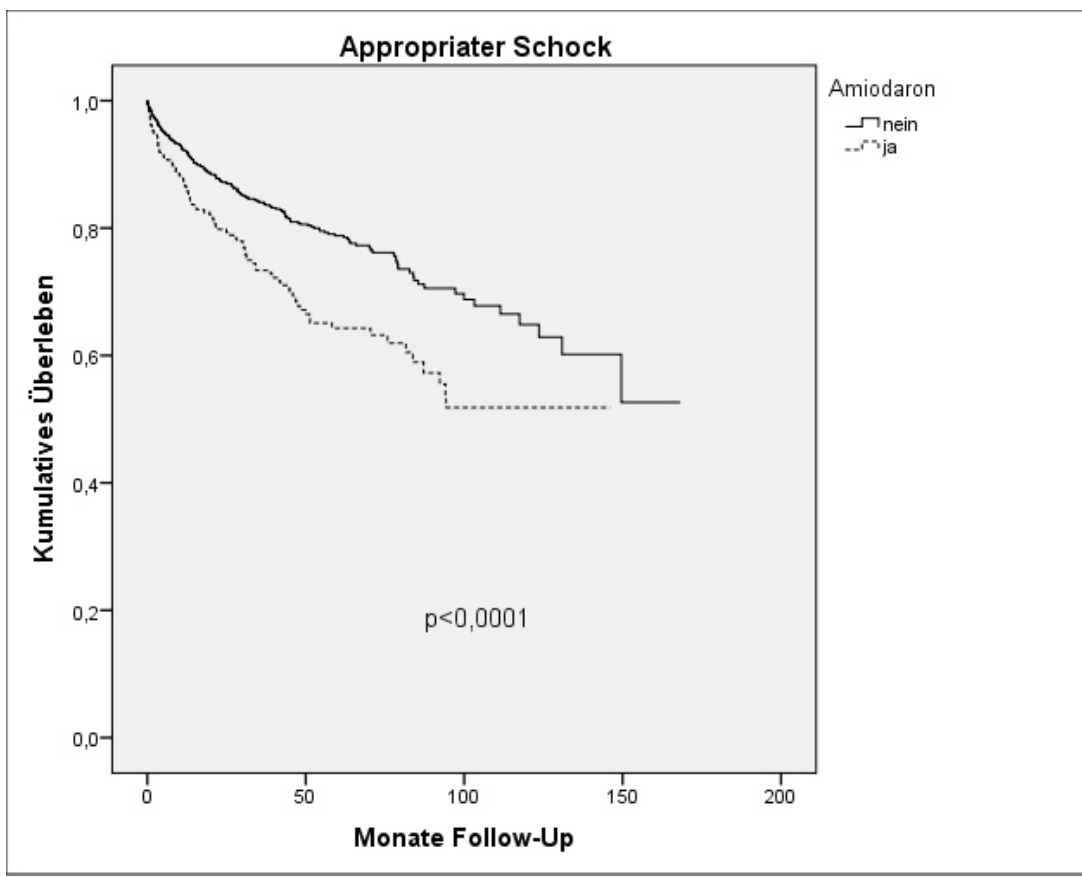

\begin{tabular}{l|c|c|c|c} 
Monate & 0 & 50 & 100 & 150 \\
\hline kein Amiodaron & 1003 & 407 & 74 & 6 \\
\hline Amiodaron & 260 & 97 & 24 & 0
\end{tabular}

\subsection{Analyse von Prädiktoren für inappropriate ICD-Schocks}

Analog der Prädiktor-Analyse für appropriate Therapien wurden die gleichen CoxRegressionsanalysen für das Auftreten von inappropriaten Schocks durchgeführt. Es wurden die gleichen 23 Variablen untersucht (siehe Tabelle 17).

\subsubsection{Univariate Cox-Regressionsanalyse}

In der jeweils einzeln durchgeführten univariaten Cox-Regressionsanalyse zeigten fünf Parameter einen $p$-Wert $\leq 0,10$ und wurden in die multivariate Analyse einbezogen. Diese sind in Tabelle 17 hervorgehoben. 
Tabelle 17: Univariate Cox-Regressionsanalyse für inappropriate Schocks

\begin{tabular}{|c|c|c|c|}
\hline & p-Wert & Hazard Ratio & 95\% Konfidenzintervall \\
\hline Vorhofflimmern & 0,002 & 1,936 & $1,275-2,942$ \\
\hline Implantationsalter > $67 \mathrm{~J}$. & 0,006 & 0,526 & $0,333-0,831$ \\
\hline männliches Geschlecht & 0,756 & 1,087 & $0,642-1,841$ \\
\hline NYHA-Stadium III / IV & 0,214 & 1,354 & $0,839-2,185$ \\
\hline LVEF $<30 \%$ & 0,445 & 0,846 & $0,550-1,300$ \\
\hline Primärprophylaxe & 0,559 & 0,882 & $0,578-1,344$ \\
\hline KHK & 0,018 & 0,608 & $0,403-0,917$ \\
\hline DCM & 0,240 & 1,284 & $0,846-1,948$ \\
\hline Z.n. ACVB & 0,772 & 0,931 & $0,574-1,510$ \\
\hline Z.n. Myokardinfarkt & 0,564 & 0,883 & $0,579-1,347$ \\
\hline Diabetes mellitus & 0,345 & 0,780 & $0,466-1,306$ \\
\hline COPD & 0,796 & 1,081 & $0,598-1,954$ \\
\hline arterielle Hypertonie & 0,017 & 0,546 & $0,332-0,898$ \\
\hline Hyperlipoproteinämie & 0,963 & 0,990 & $0,638-1,534$ \\
\hline ACE-Hemmer & 0,848 & 0,942 & $0,511-1,737$ \\
\hline Betablocker & 0,386 & 1,409 & $0,649-3,058$ \\
\hline Statin & 0,126 & 0,716 & $0,467-1,098$ \\
\hline Diuretika & 0,630 & 1,127 & $0,693-1,835$ \\
\hline Sotalol & 0,515 & 0,049 & $0,001-437,626$ \\
\hline Amiodaron & 0,909 & 1,030 & $0,617-1,720$ \\
\hline Kreatinin-Wert erhöht & 0,568 & 0,884 & $0,579-1,350$ \\
\hline NT-proBNP > 400 pg/ml & 0,009 & 0,249 & $0,087-0,711$ \\
\hline QRS-Breite > $120 \mathrm{~ms}$ & 0,612 & 0,865 & $0,493-1,516$ \\
\hline
\end{tabular}

Abkürzungen wie in Tabelle 15

\subsubsection{Multivariate Cox-Regressionsanalyse}

Von den 1230 vollständigen Datensätzen, die in der multivariaten Analyse untersucht wurden, war in 79 Fällen das Ereignis "inappropriater Schock“ eingetreten.

Die schrittweise Rückwärts-Selektion führte zu zwei signifikanten Ergebnissen: Vorhofflimmern $(p=0,002)$ bedeutete ein mehr als zweifach erhöhtes Risiko, inappropriaten ICD-Therapien ausgesetzt zu sein, während sich bei einem Implantationsalter 
über 67 Jahren $(p=0,009)$ ein nahezu halbiertes Risiko für solche Ereignisse zeigte. Tabelle 18 zeigt diese Ergebnisse in einem multivariaten Cox-Regressionsmodell.

Tabelle 18: Multivariates Cox-Regressionsmodell für inappropriate Schocks

\begin{tabular}{lrrr}
\hline & p-Wert & Hazard Ratio & 95\% Konfidenzintervall \\
\hline Vorhofflimmern & 0,002 & 2,015 & $1,292-3,142$ \\
Implantationsalter $>67 \mathrm{~J}$. & 0,009 & 0,520 & $0,317-0,851$ \\
\hline
\end{tabular}

\subsubsection{Kaplan-Meier-Überlebenskurven}

Die beiden Einflussgrößen Vorhofflimmern und Implantationsalter für das Auftreten inappropriater ICD-Schocks sind in den Abbildungen 14 und 15 als Kaplan-MeierAnalysen dargestellt.

Abbildung 14: Inappropriater Schock (Vorhofflimmern)

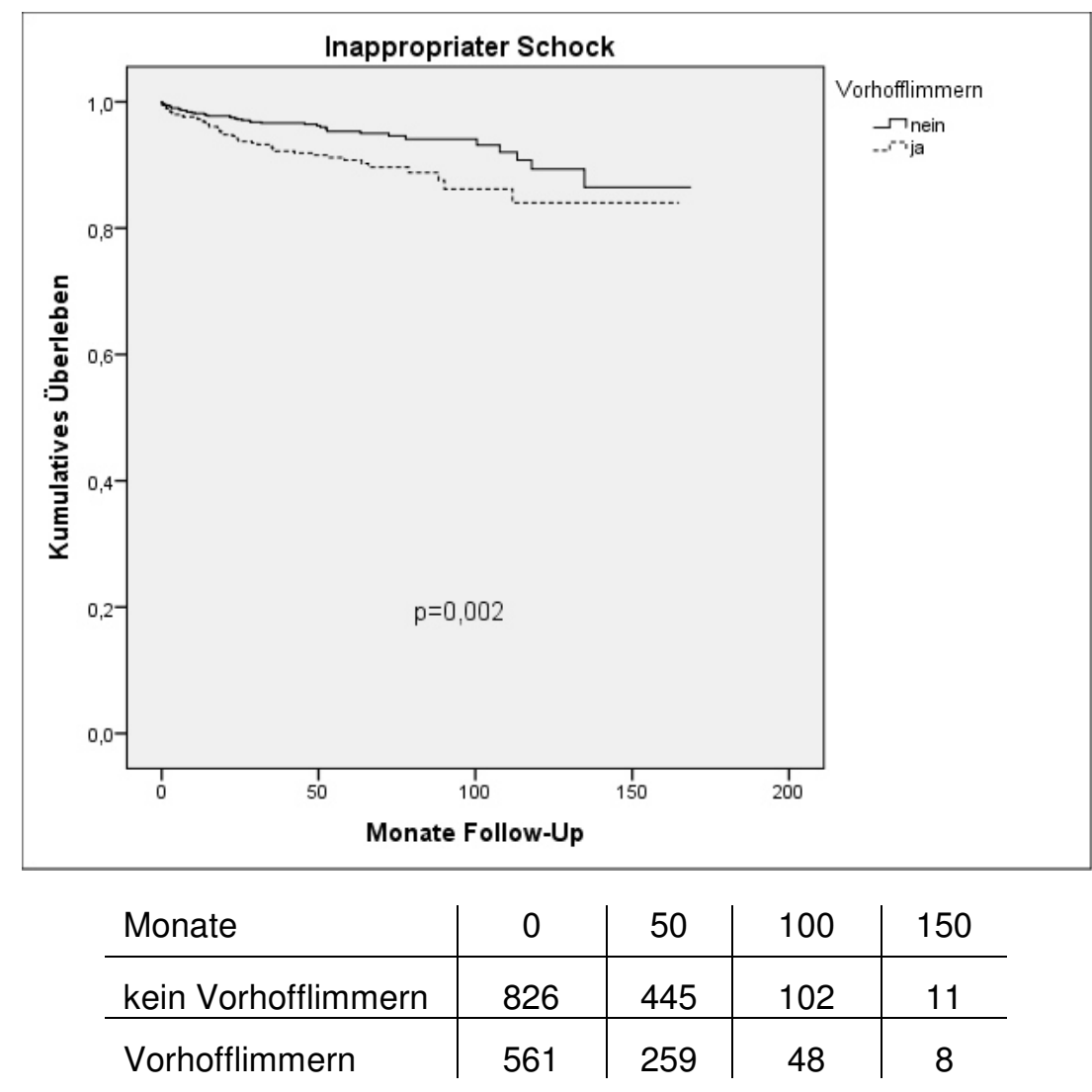


Abbildung 15: Inappropriater Schock (Implantationsalter)

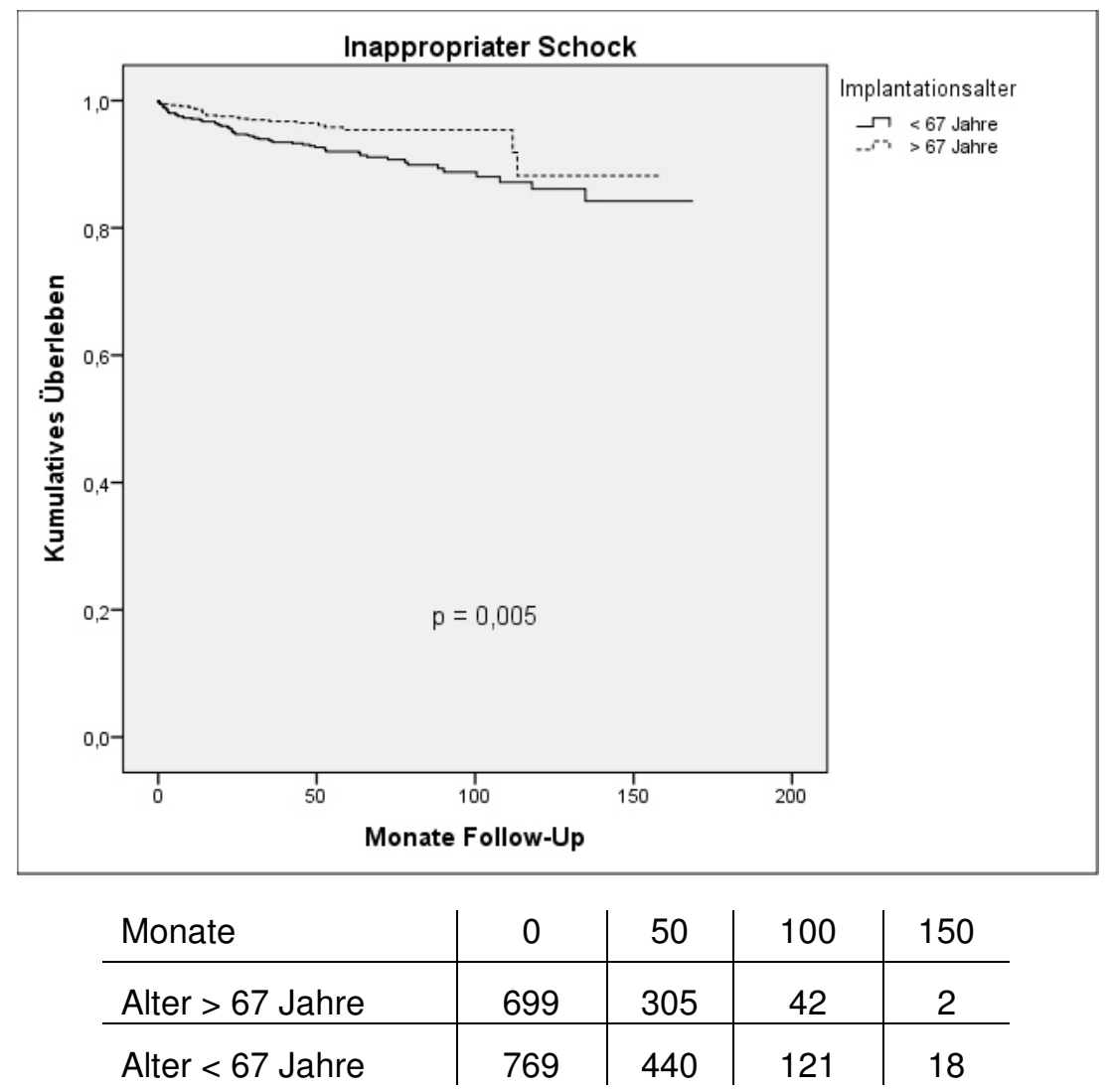

\subsection{Tod ohne vorherige appropriate ICD-Therapie}

Von den 1304 Patienten, über die Daten zur eventuellen ICD-Therapie vorliegen, sind 265 Patienten (20\%) ohne bekannte vorherige appropriate ICD-Schocks verstorben. 24 Patienten (2\%) erhielten nur inappropriate Schocks, bei den restlichen 241 Patienten (18\%) sind keine Schocks bekannt. Dem gegenüber stehen 331 Patienten $(25 \%)$ mit im Vorfeld dokumentierter appropriater Therapie.

\subsubsection{Univariate Cox-Regressionsanalyse}

Um auch hier eventuelle Einflussfaktoren für das Ereignis „Tod ohne vorherige appropriate ICD-Therapie" zu finden, wurden die bereits verwendeten 23 Parameter einer weiteren univariaten Regressionsanalyse unterzogen. Zwölf Ergebnisse mit einem $p$-Wert $\leq 0,10$ sind in Tabelle 19 hervorgehoben. 
Tabelle 19: Univariate Cox-Regressionsanalyse für Tod ohne vorherige appropriate Therapie

\begin{tabular}{|c|c|c|c|}
\hline & p-Wert & Hazard Ratio & 95\% Konfidenzintervall \\
\hline Vorhofflimmern & $<0,001$ & 1,632 & $1,268-2,101$ \\
\hline Implantationsalter > $67 \mathrm{~J}$. & $<0,001$ & 2,955 & $2,283-3,824$ \\
\hline männliches Geschlecht & 0,335 & 1,169 & $0,851-1,608$ \\
\hline NYHA-Stadium III / IV & $<0,001$ & 2,747 & $1,999-3,775$ \\
\hline LVEF $<30 \%$ & 0,007 & 1,470 & $1,114-1,940$ \\
\hline Primärprophylaxe & $<0,001$ & 1,718 & $1,330-2,220$ \\
\hline KHK & 0,008 & 1,461 & $1,106-1,930$ \\
\hline DCM & 0,883 & 0,981 & $0,757-1,271$ \\
\hline Z.n. ACVB & 0,189 & 1,204 & $0,913-1,587$ \\
\hline Z.n. Myokardinfarkt & 0,227 & 1,164 & $0,910-1,490$ \\
\hline Diabetes mellitus & 0,003 & 1,516 & $1,152-1,994$ \\
\hline COPD & $<0,001$ & 2,199 & $1,629-2,970$ \\
\hline arterielle Hypertonie & 0,165 & 1,314 & $0,894-1,931$ \\
\hline Hyperlipoproteinämie & 0,266 & 1,164 & $0,891-1,520$ \\
\hline ACE-Hemmer & 0,831 & 0,960 & $0,663-1,392$ \\
\hline Betablocker & 0,211 & 0,788 & $0,543-1,144$ \\
\hline Statin & 0,587 & 0,929 & $0,713-1,211$ \\
\hline Diuretika & $<0,001$ & 2,317 & $1,607-3,342$ \\
\hline Sotalol & 0,240 & 0,047 & $0,001-7,687$ \\
\hline Amiodaron & 0,967 & 1,007 & $0,736-1,377$ \\
\hline Kreatinin-Wert erhöht & $<0,001$ & 1,370 & $1,196-1,570$ \\
\hline NT-proBNP > $400 \mathrm{pg} / \mathrm{ml}$ & 0,023 & 10,087 & $1,377-73,889$ \\
\hline QRS-Breite > $120 \mathrm{~ms}$ & $<0,001$ & 1,876 & $1,332-2,642$ \\
\hline
\end{tabular}

Abkürzungen wie in Tabelle 15

\subsubsection{Multivariate Cox-Regressionsanalyse}

Für die multivariate Analyse mit schrittweiser Rückwärts-Selektion wurden 834 vollständige Datensätze untersucht, wobei das Ereignis „Tod ohne vorherige appropriate ICD-Therapie" in 153 Fällen eingetreten war. Es zeigte sich bei drei Parametern ein statistisch signifikanter Einfluss (siehe Tabelle 20). Sowohl ein Erstimplantationsalter 
von über 67 Jahren als auch eine fortgeschrittene Herzinsuffizienz (NYHA-Stadium III / IV) und die Einnahme von Diuretika sind mit einem erhöhten Risiko für ein Versterben assoziiert, ohne dass es zu einer vorherigen appropriaten Therapie des ICD kam.

Tabelle 20: Multivariates Cox-Regressionsmodell für Tod ohne vorherige appropriate Therapie

\begin{tabular}{lrrr}
\hline & p-Wert & Hazard Ratio & 95\% Konfidenzintervall \\
\hline Implantationsalter $>$ 67 J. & $<0,001$ & 2,726 & $1,896-3,919$ \\
NYHA-Stadium III / IV & $<0,001$ & 2,186 & $1,498-3,191$ \\
Diuretika & 0,014 & 1,916 & $1,141-3,217$ \\
\hline
\end{tabular}

\subsubsection{Kaplan-Meier-Überlebenskurven}

Die Abbildungen 16 - 18 zeigen die grafischen Darstellungen der ermittelten Prädiktoren als Kaplan-Meier-Diagramme.

Abbildung 16: Tod ohne vorherige appropriate Therapie (Implantationsalter)

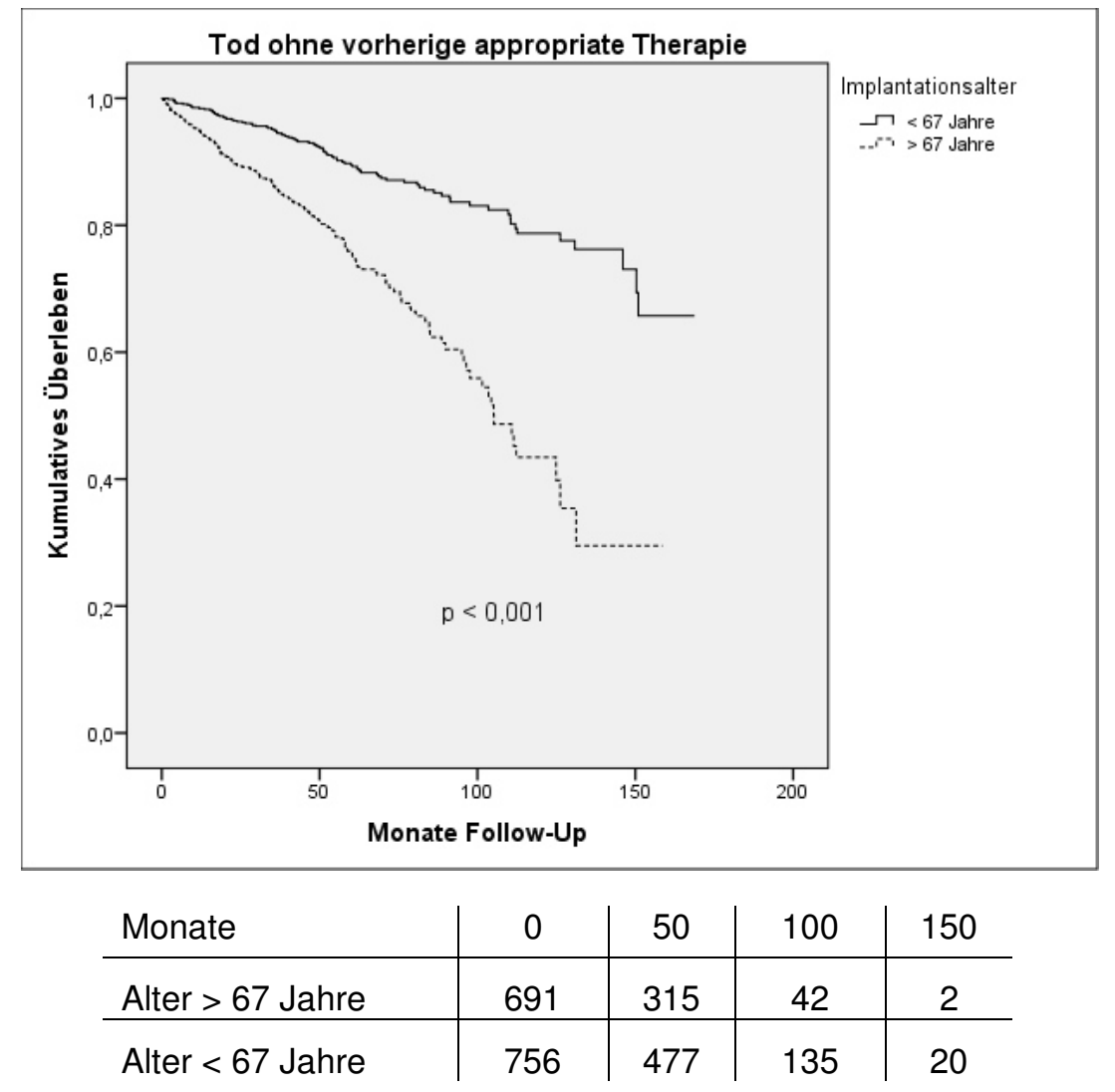


Abbildung 17: Tod ohne vorherige appropriate Therapie (NYHA)

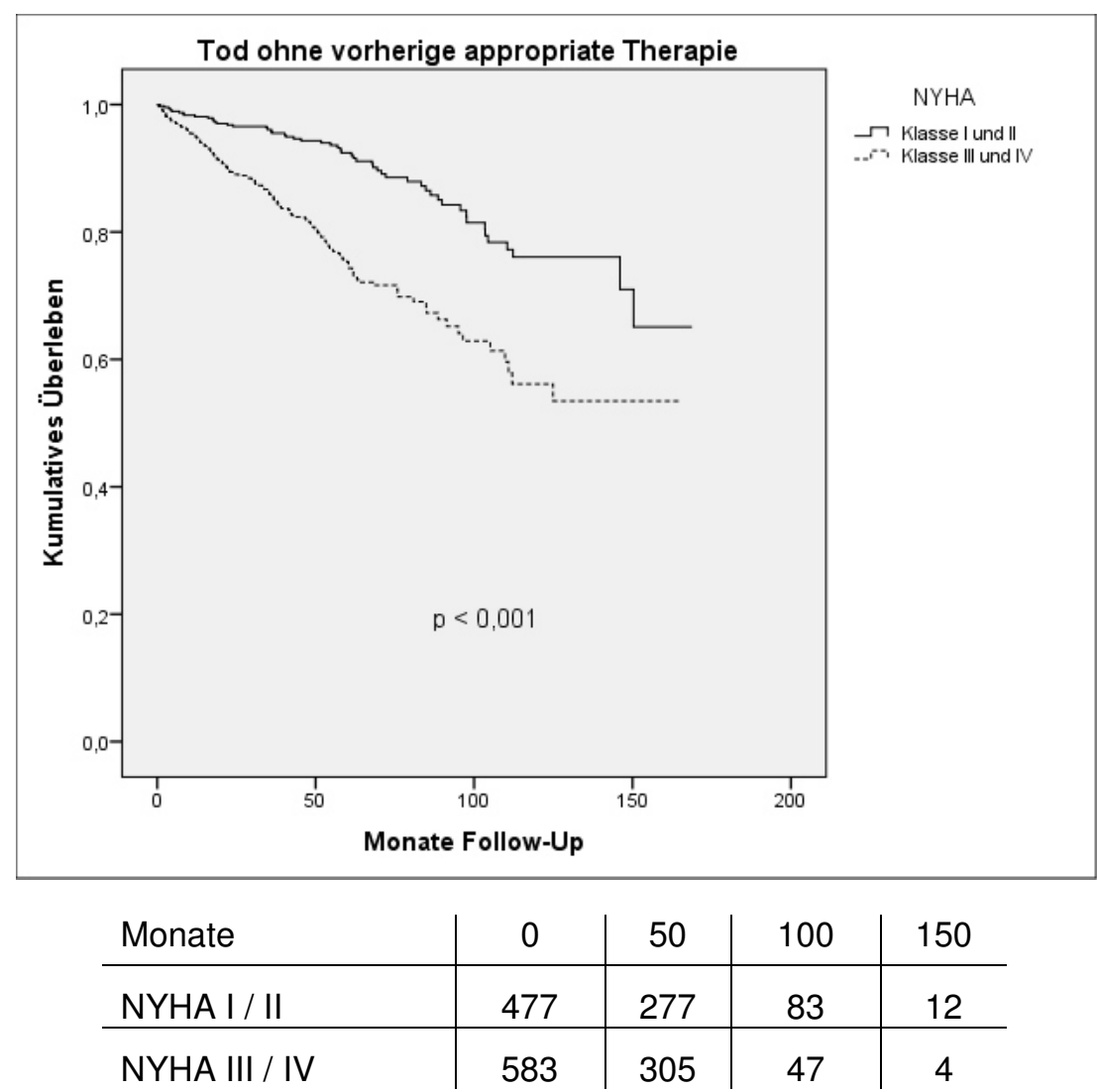

Abbildung 18: Tod ohne vorherige appropriate Therapie (Diuretika)

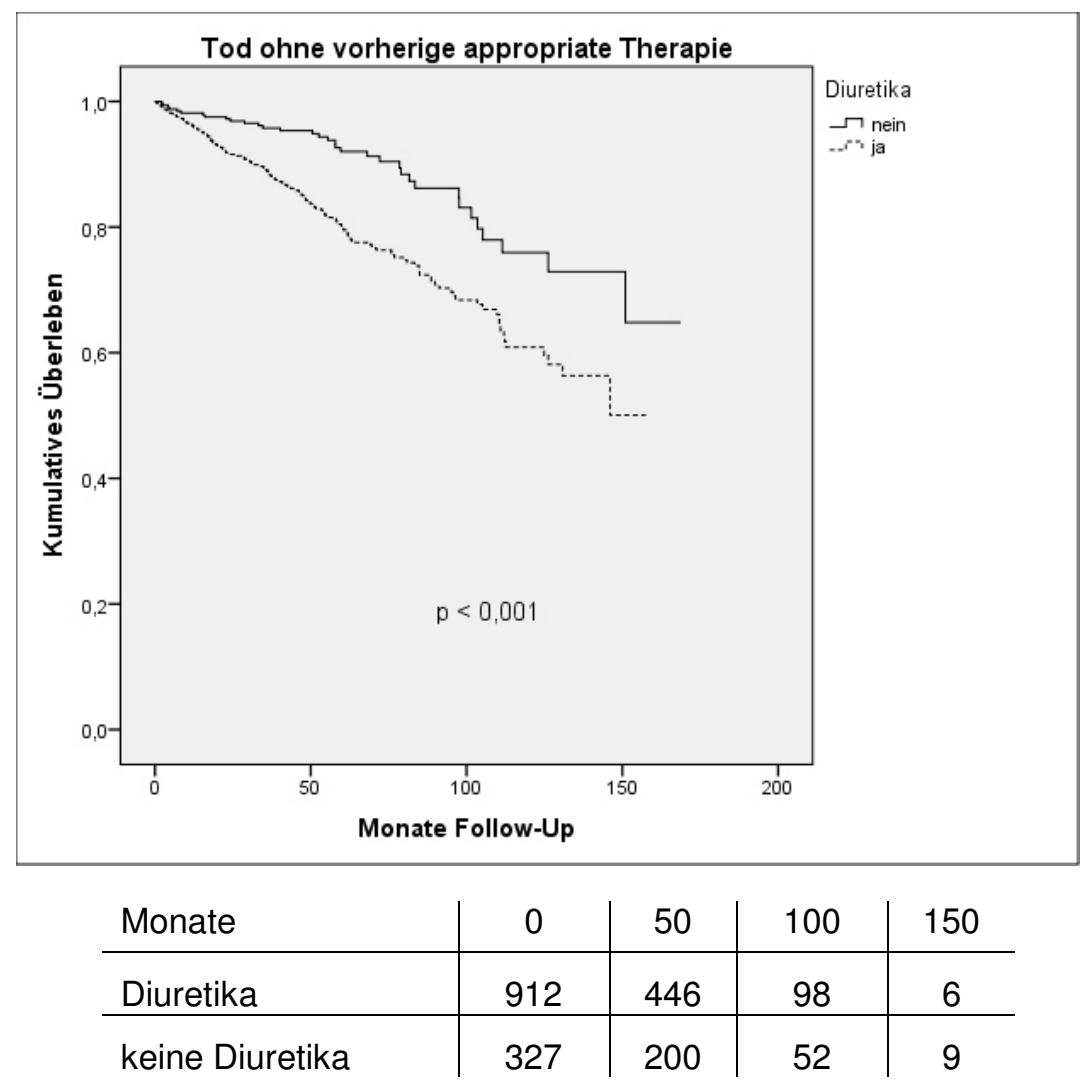




\section{Diskussion}

\subsection{Prädiktoren appropriater ICD-Schocks}

In der untersuchten Population erhielten insgesamt 25\% der Patienten (331/1304), bei denen Daten zur ICD-Therapie vorlagen, appropriate ICD-Schocks. Dabei zeigten sich als unabhängige Faktoren für das Auftreten von appropriaten ICD-Schocks ein männliches Geschlecht (HR: 1,7; 95\% Cl: 1,1 - 2,4; $\mathrm{p}=0,008)$ und eine Amiodaroneinnahme zum Zeitpunkt der Implantation (HR: 1,6;95\% Cl: 1,2 - 2,1; $\mathrm{p}=$ $0,002)$. Eine primärprophylaktische Implantationsindikation war im Vergleich zur Sekundärprophylaxe hingegen mit einem geringeren Risiko für appropriate Therapien assoziiert (HR: 0,7; 95\% Cl: 0,6-0,9; $p=0,014$ ).

Männer haben ein höheres Risiko für kardiovaskuläre Krankheiten gegenüber Frauen (Bays 2011). In einer Untersuchung des finnischen Registers für Todesursachen im Zeitraum von 1986 bis 2009 wurden mehr als 1 Million Todesfälle betrachtet, wobei sich für Männer ein alterskorrigiertes um 80\% erhöhtes Risiko, an einer kardiovaskulären Krankheit zu sterben, zeigte (Mikkola et al. 2013).

In der untersuchten Population von 1484 ICD-Patienten waren 81\% männlich. Der gefundene Zusammenhang von appropriater ICD-Therapie beziehungsweise dem Auftreten ventrikulärer Tachyarrhythmien und männlichem Geschlecht wird auch in anderen Studien beschrieben (Klein et al. 2003, Wijers et al. 2013).

Ghanbari et al. (2009) führten eine Metaanalyse von fünf randomisierten klinischen Studien zur Primärprophylaxe des plötzlichen Herztodes mittels ICD durch. Dabei zeigte sich keine signifikante Reduktion der Gesamtmortalität in der weiblichen Studienpopulation.

Eine weitere Metaanalyse von Studien mit insgesamt 7229 Patienten, davon 22\% Frauen, zeigte vergleichbare Mortalitätsraten beider Geschlechter, während Frauen signifikant weniger appropriate Therapien erhalten. Dies könnte ein Hinweis auf den geringeren Anteil von plötzlichem Herztod an der Gesamtmortalität bei Frauen sein und den geringeren Nutzen einer ICD-Implantation in der weiblichen Population erklären (Santangeli et al. 2010).

In einer im Jahr 2013 veröffentlichten Studie von Bhavnani et al. konnte bestätigt werden, dass Frauen eine signifikant niedrigere Anzahl appropriater ICD-Therapien erhielten. Die Mortalitätsrate lag in beiden Gruppen bei $25 \%$. Um einen rein durch das Geschlecht bedingten Unterschied zu untersuchen, wurden je 291 Frauen und 
Männer mit gleichen demographischen und klinischen Charakteristika untersucht (Bhavnani et al. 2013). Diese Daten unterstützen die Vermutung einer geringeren Anzahl arrhythmisch bedingter Todesfälle bei Frauen im Vergleich zu Männern.

Hierfür werden unterschiedliche Ursachen diskutiert. So fanden Haigney et al. (2009) in einer Untersuchung von 142 Frauen der MADIT-II-Population heraus, dass bei innen eine erhöhte Repolarisationsdauer und Herzfrequenzvariabilität kein erhöhtes Arrhythmierisiko birgt.

Zusätzlich hat eine prospektive Multicenter-Studie mit 6021 Patienten (21\% Frauen) eine höhere Komplikationsrate bei Frauen zu den Zeitpunkten 45 Tage beziehungsweise ein Jahr nach ICD-Implantation ergeben, während weniger appropriate Schocks auftraten (MacFadden et al. 2012).

Ähnliche Prädiktoren für das Auftreten appropriater ICD-Therapien wie in der vorliegenden Arbeit fanden auch Wijers et al. (2013) in einer Untersuchung von 1075 Patienten. Männliches Geschlecht, eine sekundärprophylaktische Implantation, ischämische Kardiomyopathie und eine LVEF $\leq 25 \%$ waren dort mit einem höheren Risiko für appropriate Schocks assoziiert.

Dass Patienten mit primärprophylaktisch implantierten ICD weniger Schocks erhielten, zeigten auch Koller et al. (2008). Die Wahrscheinlichkeit für appropriate Schocks war in der Gruppe mit sekundärprophylaktischer Implantation fast doppelt so groß. Weitere Prädiktoren waren eine erniedrigte LVEF und höheres Alter.

Amiodaron hat komplexe elektrophysiologische Wirkmechanismen, unter anderem verlängert sich die atriale und ventrikuläre Repolarisationsdauer durch Blockade von myokardialen Kaliumkanälen. Amiodaron wird erfolgreich eingesetzt zur Prävention von ventrikulären Tachyarrhythmien und zur Prophylaxe des plötzlichen Herztodes.

Durch direkte Effekte am Sinusknoten wird die Herzfrequenz gesenkt und eine Verlängerung der AV-Überleitungszeit bewirkt den positiven Einfluss auf supraventrikuläre Arrhythmien (Connolly 1999). Diese Mechanismen können einen Beitrag zur Verhinderung inappropriater Schocks leisten. Alter et al. (2005) beschreiben die Assoziation von Amiodaroneinnahme zum Implantationszeitpunkt und der Reduktion inappropriater Schocks bei supraventrikulären Tachyarrhythmien.

Im Vergleich zu anderen antiarrhythmischen Medikamenten sind die kardialen $\mathrm{Ne}$ benwirkungen gering, aber es gibt zahlreiche nicht-kardiale unerwünschte Arzneimittelwirkungen wie pulmonale und hepatische Toxizität, thyreoidale Dysfunktionen, 
Keratopathien und periphere Neuropathien. Deshalb und auch aufgrund der langen Eliminationshalbwertszeit von bis zu 60 Tagen bedarf der Einsatz des Medikaments daher regelmäßiger Kontrollen.

Die von Hohnloser et al. (2006) beschriebene Erhöhung der Defibrillationsschwelle in einer Subgruppe von Patienten der OPTIC-Studie (Optimal Pharmacological Therapy in Cardioverter Defibrillator Patients) war in der Amiodaron-Gruppe zwar signifikant erhöht, aber nie größer als die festgelegte Sicherheitsgrenze von 10 Joule, sodass kein Interventionsbedarf bestand.

Der in der vorliegenden Auswertung gefundene Zusammenhang von erhöhtem Schockrisiko bei Einnahme von Amiodaron zum Implantationszeitpunkt bestätigte sich nicht in großen randomisierten Studien.

In der CASCADE-Studie (Cardiac Arrest in Seattle: Conventional Versus Amiodarone Drug Evaluation) wurde an 228 Patienten mit überlebtem Kammerflimmern gezeigt, dass in der Gruppe der mit Amiodaron Behandelten signifikant weniger ICD-Schocks aufgetreten sind als bei der Therapie mit Klasse-I-Antiarrhythmika (Greene 1993).

Connolly et al. (2006) untersuchten in der OPTIC-Studie, ob Amiodaron zur Reduzierung von ICD-Schocks beiträgt. In der prospektiven Multicenter-Studie wurden 412 Patienten mit sekundärprophylaktischem ICD drei Gruppen zugewiesen und erhielten entweder Amiodaron plus Betablocker, Sotalol oder einen Betablocker. Nach einem Jahr zeigte sich in der mit Amiodaron plus Betablocker behandelten Gruppe eine relative Risikoreduktion für sowohl appropriate als auch inappropriate Schocks von $57 \%$ verglichen mit Sotalol sowie 73\% im Vergleich mit alleiniger Betablockertherapie. 18\% der mit Amiodaron behandelten Patienten hatten das Medikament aufgrund pulmonaler oder thyreoidaler Nebenwirkungen beziehungsweise symptomatischer Bradykardien abgesetzt.

Ein wichtiger Unterschied beider Studien zu der hier untersuchten Population ist, dass ausschließlich Patienten mit Sekundärprophylaxe eingeschlossen wurden und die Ergebnisse nur bedingt übertragbar sind auf Patienten mit primärprophylaktisch implantiertem ICD. Aber auch in der vorliegenden Untersuchung hatten die mit Amiodaron behandelten Patienten den ICD häufiger zur Sekundärprophylaxe erhalten als in der Gesamtpopulation (65\% versus 46\%).

Die Erhöhung des Schockrisikos durch Amiodaron kann also nicht nur aufgrund der Implantationsindikation erklärt werden, sondern ist mit hoher Wahrscheinlichkeit dadurch bedingt, dass die betroffenen Patienten aufgrund stattgehabter Arrhythmien 
und Gefahr von Rezidiven zum Zeitpunkt der ICD-Implantation mit Amiodaron behandelt wurden. Der statistische Zusammenhang könnte deswegen nur das höhere arrhythmische Potential widerspiegeln, statt einen ursächlichen Zusammenhang zu beschreiben.

Dass Vorhofflimmern mit ventrikulären Tachykardien assoziiert sein kann, zeigte sich in mehreren Untersuchungen.

In einer prospektiven Studie mit 229 Patienten, von denen bei 17\% zum Implantationszeitpunkt ein Vorhofflimmern vorlag, zeigte sich für diese Patienten im durchschnittlichen Follow-Up von 20 Monaten ein 1,8-fach erhöhtes Risiko, appropriate Therapien zu erhalten. Bemerkenswert ist dabei, dass nicht etwa eine schnelle Überleitung des Vorhofflimmerns die ventrikulären Tachyarrhythmien auslöst, sondern komplexe elektrophysiologische Prozesse zur elektrischen Instabilität im Ventrikelmyokard führen können (Grönefeld et al. 2000)

Das Auftreten sogenannter „dualer Tachykardien“, das heißt ventrikulärer Tachyarrhythmien, denen entweder eine atriale Tachykardie (AT) oder ein Vorhofflimmern (AF) vorausgeht, beschreiben Stein et al. (2002). Insgesamt 9\% aller detektierten Episoden ventrikulärer Tachyarrhythmien erfüllten die Kriterien. Untersucht wurden 537 Patienten mit Zweikammer-ICD und mindestens 2 Episoden von AT / AF im vorangegangenen Jahr, ein permanentes Vorhofflimmern war ein Ausschlusskriterium. Abgegebene ICD-Therapien beendeten zwar alle Fälle von ventrikulären Tachyarrhythmien, aber nur 40\% der AT / AF-Episoden. Bei diesen Patienten kam es nach signifikant kürzerer Zeit zum Wiederauftreten von ventrikulären Tachykardien oder Kammerflimmern.

Die PROFIT-Studie (Prospective Analysis of Risk Factors for appropriate ICD Therapy) ermittelte als unabhängige Prädiktoren für das Auftreten ventrikulärer Tachyarrhythmien ebenfalls ein permanentes Vorhofflimmern sowie eine Ejektionsfraktion < 40\% und eine QRS-Breite > 150 ms. Bei Vorliegen von zwei oder mehr dieser Risikofaktoren betrug das 2-Jahres-Risiko 100\%. Von 250 Patienten hatten 92\% den ICD zur Sekundärprophylaxe erhalten, es wurden nur 46 Patienten mit ventrikulären Tachyarrhythmien in die Prädiktor-Analyse einbezogen (Klein et al. 2006).

In der hier beschriebenen Population bestand zum Implantationszeitpunkt bei insgesamt 564 Patienten (40\%) ein Vorhofflimmern. Es zeigte sich lediglich in der univariaten Analyse ein erhöhtes Risiko für appropriate ICD-Schocks bei Vorliegen von Vor- 
hofflimmern (HR: 1,3; 95\% Cl: 1,0 - 1,6; $\mathrm{p}=0$ 0,039). In der multivariaten Analyse wurde keine statistische Signifikanz erreicht.

Eine weitere prospektive Studie zur Prädiktion von appropriaten Schocks anhand klinischer Charakteristika ist die von Whang et al. (2004) ausgewertete TOVA-Studie (Triggers Of Ventricular Arrhythmias), in der sich bei 502 Patienten ein NYHAStadium III und eine LVEF < $20 \%$ als unabhängige Prädiktoren für appropriate Schocks zeigten. Sowohl das Vorliegen einer Herzinsuffizienz als auch eine verringerte LVEF waren in dieser Analyse nicht mit der Häufigkeit von appropriaten ICDTherapien assoziiert.

Die Analyse von Prädiktoren für appropriate ICD-Schocks dient nicht nur der Abschätzung, welche Patienten am meisten von einer ICD-Implantation profitieren. Sie ist auch nötig, um zu identifizieren, wer ein besonders hohes Risiko für arrhythmische Ereignisse hat, um gegebenenfalls die Therapie anzupassen, sofern das möglich ist, wie zum Beispiel eine intensivierte Herzinsuffizienzbehandlung.

Bei zwei der drei in der vorliegenden Arbeit beschriebenen Variablen (Geschlecht und Implantationsindikation) ist dies nicht möglich. Auch die Interventionsmöglichkeiten bezüglich der Amiodarontherapie sind gering. Es wurde zudem nur die Amiodaroneinnahme zum Implantationszeitpunkt betrachtet und es liegen keine Daten über eine eventuelle Weiterführung im Follow-Up vor.

Um die Häufigkeit von appropriaten Schocks zu reduzieren, hat sich antitachykardes Pacing als sehr wirksam gezeigt und war daher in der hier beschriebenen ICDPopulation standardmäßig aktiviert. Bei insgesamt 288 Patienten (22\%) kam es zu einer appropriaten ATP-Abgabe, bei 49 Patienten (4\%) zu inappropriater Therapie mittels ATP, am häufigsten aufgrund supraventrikulärer Arrhythmien.

Klein et al. (2003) beschreiben die Erfolgsraten bei 149 Patienten der AVID-Studie, die ATP dauerhaft aktiviert hatten. Es wurden 67\% aller VT-Episoden erfolgreich terminiert, bei schnellen VT-Episoden > 187 bpm sogar $84 \%$. Insgesamt kam es nur bei 4\% aller Episoden zu einer Akzeleration der Tachyarrhythmien.

Zu ähnlichen Ergebnissen kommen Wathen et al. (2001), die zeigen konnten, dass bei 220 Patienten mit koronarer Herzkrankheit insgesamt 77\% aller aufgetretenen schnellen VT-Episoden (188 bis 250 bpm) mittels ATP terminiert werden konnten.

Um diese Ergebnisse zu verifizieren, wurden in der prospektiven PainFREE Rx IIStudie (Pacing Fast VT Reduces Shock Therapies) 634 Patienten in 2 Gruppen ge- 
teilt, wobei die initiale Therapie für FVT-Episoden entweder ATP oder Schock war (Wathen et al. 2004). Nach durchschnittlich 11 Monaten Follow-Up konnten 81\% der FVT-Episoden erfolgreich durch antitachykardes Pacing beendet werden, ohne dass es zu einer Zunahme von akzelerierten Arrhythmien, Synkopen oder Fällen von plötzlichem Herztod kam. Die Lebensqualität stieg in der Gruppe mit ATP deutlicher an als in der Vergleichsgruppe. Somit trägt ATP wirksam zur Reduktion von Schocks bei und sollte auch für schnelle ventrikuläre Tachykardien initiale Therapie der Wahl sein.

Bei nicht erfolgreichem ATP oder für den Fall einer Akzeleration der Arrhythmie muss zeitnah die Abgabe eines Schocks erfolgen. Um diese Zeit möglichst gering zu halten, gibt es die Möglichkeit der ATP-Therapie, während der ICD für den Schock auflädt. Die Effektivität dieser Methode zeigen Schoels et al. (2007). Bei 421 untersuchten Patienten konnte in $69 \%$ der mit ATP behandelten Arrhythmieepisoden ein Schock verhindert werden.

\subsection{Prädiktoren inappropriater ICD-Schocks}

Erfolgte eine Therapieabgabe des ICD aufgrund anderer Ursachen als von ventrikulären Tachykardien oder Kammerflimmern, so wurde dies als inappropriat gewertet (Daubert et al. 2008). Insgesamt 10\% der Patienten (134/1304) erhielten inappropriate Schocks. Die Ursachen der inappropriaten Therapien waren Vorhofflimmern (29\%), supraventrikuläre Tachykardien (26\%) und in 35\% der Fälle fehlerhaftes Sensing beziehungsweise mechanische Defekte der Sonden. Bei 10\% der Patienten konnte aufgrund fehlender Daten keine Einordnung der Ursache erfolgen.

Bestand bei der ICD-Implantation ein Vorhofflimmern, so ist das Risiko, im Verlauf inappropriate Schocks zu erhalten, doppelt so groß im Vergleich zu Patienten mit Sinusrhythmus (HR: 2,0;95\% Cl: 1,3-3,1; $p=0,002$ ). Halbiert ist das Risiko für inappropriate Schocks hingegen bei Patienten, die den ICD im Alter von über 67 Jahren erhalten hatten (HR: 0,5;95\% $\mathrm{Cl}: 0,3-0,9 ; \mathrm{p}=0,009$ ).

Da Vorhofflimmern mit schneller Überleitung auf den Ventrikel eine der häufigsten Ursachen für inappropriate Schocks darstellt, war dieser Zusammenhang erwartet worden. Dies zeigte sich auch in den bereits beschriebenen Studien von Grönefeld et al. (2000) und Wijers et al. (2013).

In einer Untersuchung von 719 Patienten der MADIT-II-Studie waren neben Vorhofflimmern weitere Prädiktoren für inappropriate Schocks ein Nikotinkonsum, ein dias- 
tolischer Blutdruck > $80 \mathrm{~mm} \mathrm{Hg}$ und vorherige appropriate ICD-Schocks (Daubert et al. 2008). Bis auf Vorhofflimmern wurden diese Prädiktoren hier nicht untersucht, da hierzu keine Daten vorlagen.

Da Vorhofflimmern nicht nur mit inappropriaten Schocks assoziiert ist, sondern auch mit zahlreichen klinischen Komplikationen (Herzinsuffizienz, Thrombembolien) und einer bis zu zweifach erhöhten Mortalität, wie in einer Untersuchung von 4731 Patienten der Framingham-Studie über einen Zeitraum von 38 Jahren gezeigt werden konnte (Kannel et al. 1998), kommt diesem Parameter in der klinischen Praxis eine besondere Bedeutung zu.

In einer 2011 veröffentlichten Studie von van Rees et al. traten bei $13 \%$ von 1544 Patienten nach einem mittleren Follow-Up von 41 Monaten inappropriate Schocks auf. Prädiktoren dafür mit zweifach erhöhtem Risiko waren ein Alter $<70$ Jahren und Vorhofflimmern. Außerdem waren die Nichteinnahme von Statinen und ein vorhergehender appropriater Schock mit häufigeren inappropriaten Schocks assoziiert. Die untersuchte Population war von demographischen und klinischen Charakteristika weitgehend mit den in der vorliegenden Arbeit untersuchten Patienten vergleichbar, lediglich Vorhofflimmern lag seltener vor (23\% versus $40 \%)$. Ein weiterer Unterschied war die geringere Anzahl der inappropriaten Therapien, die durch fehlerhaftes Sensing bedingt waren (12\% versus $35 \%$ ). Die vergleichbare Populationsgröße, das lange Follow-Up sowie ähnliche Ergebnisse könnten aber ein Hinweis sein, dass diese Prädiktoranalyse eher zutreffend ist als in anderen, kleineren Studien.

Den positiven Einfluss von Statinen beschreiben auch Bhavnani et al. (2008) in einer prospektiven Studie mit 1445 Patienten. Die Einnahme von Statinen war sowohl mit der Reduktion der Häufigkeit von Vorhofflimmern als auch dem verminderten Risiko für inappropriate Schocks assoziiert.

In dieser Auswertung zeigte sich keine statistische Signifikanz für einen Einfluss der Statintherapie auf inappropriate Schocks (HR: 0,7; 95\% Cl: 0,5-1,1; $p=0,126$ ).

Den Zusammenhang, dass bei jüngeren Patienten mehr inappropriate ICD-Schocks auftreten, beschreiben auch Alter et al. (2005) in einer Untersuchung von 440 Patienten. Im mittleren Nachbeobachtungszeitraum von 46 Monaten traten bei $12 \%$ der Patienten inappropriate Schocks auf. Dabei war das durchschnittliche Alter mit 48 versus 58 Jahren signifikant unterschiedlich.

Eine mögliche Erklärung könnte sein, dass Patienten mit Sondendefekten, die in den beschriebenen Studien wie auch in der hier untersuchten Population eine wichtige 
Ursache inappropriater Schocks darstellen, jünger sind. Kleemann et al. (2007) fanden bei einer prospektiven Untersuchung von 990 Patienten, dass diejenigen mit Sondendefekten im Durchschnitt 3 Jahre jünger und häufiger weiblich waren.

Bemerkenswert ist auch das Ergebnis einer Untersuchung von 2279 Patienten der MADIT-II- und MADIT-CRT-Studien: die 503 Frauen (22\%) hatten ein signifikant niedrigeres Risiko für inappropriate Therapien, vor allem aufgrund von niedrigerer Prävalenz von Vorhofflimmern (Tompkins et al. 2012).

Während sich bei appropriaten ICD-Schocks diese Geschlechterverteilung in dieser Untersuchung darstellte, konnte das für inappropriate Therapien nicht festgestellt werden (männliches Geschlecht HR: 1,1;95\% Cl: 0,6-1,8; $p=0,756$ ).

Da Zweikammer-ICD mit einer zusätzlichen Sonde im rechten Vorhof in der Lage sind, dort Signale wahrzunehmen, besteht die Annahme, dass diese Aggregate besser in der Lage sind, atriale und ventrikuläre Tachyarrhythmien zu unterscheiden und daher weniger inappropriate Therapien abzugeben.

Dem widersprechende Ergebnisse zeigen Deisenhofer et al. (2001) in einer prospektiven Studie mit 92 Patienten, bei denen 73 von 725 detektierten Episoden inappropriat waren. In der Gruppe mit Zweikammer-ICD traten 69\% dieser inappropriaten Episoden auf, während es bei Einkammer-ICD nur 31\% waren.

Theuns et al. (2004) zeigten in einer prospektiven Studie mit 60 Patienten, dass zusätzliche atriale Detektionsalgorithmen keinen signifikanten Vorteil brachten. 42\% aller atrialen Tachyarrhythmien wurden inappropriat behandelt. Eine stabile 1:1Überleitung und Sinustachykardie bei Vorliegen von vorzeitigen ventrikulären Erregungen waren die Hauptursachen inappropriater Detektionen. Es wurden keine Morphologiekriterien angewendet, die sich als wirksam bei der Diskrimination inappropriater Episoden erwiesen haben. Daher sind diese Ergebnisse eingeschränkt beurteilbar.

Ein fehlender Vorteil eines bestimmten Aggregattyps zeigte sich auch in der bereits genannten Population von 1544 Patienten, die van Rees et al. (2011) beschreiben. Inappropriate Therapien sind gleich häufig bei Patienten mit Ein-/Zwei- und Dreikammer-ICD.

Ruwald et al. (2013) zeigen an 704 Patienten, von denen 13\% inappropriate Therapien erhalten hatten, dass es keinen signifikanten Unterschied in den Gruppen mit Ein- oder Zweikammer-ICD gab. 
Es gibt aber auch Untersuchungen, die den Vorteil einer zusätzlichen Sonde im Vorhof zeigen. Friedman et al. (2006) beschreiben bei 400 Patienten, die in zwei Gruppen randomisiert werden, den signifikanten Vorteil von atrialen Detektionsalgorithmen in der korrekten Klassifizierung supraventrikulärer Tachykardien. Inappropriate Detektionen konnten von $40 \%$ auf $31 \%$ gesenkt werden.

Eine weitere Untersuchung, die signifikant weniger inappropriate Therapien durch Zweikammer-ICD zeigte, ist eine retrospektive Analyse von 386 Patienten (Soundarraj et al. 2006). Verglichen mit Einkammer-ICD erhielten nur 5\% versus 22\% der Patienten inappropriate Schocks.

In der vorliegenden Arbeit zeigte sich kein Unterschied zwischen Ein- oder Zweikammer-ICD und dem Auftreten von inappropriaten Schocks. Die 454 Patienten mit CRT-D hatten jedoch signifikant weniger inappropriate Schocks (im Vergleich mit Einkammer-ICD 19\% versus 39\%, $p=0,022$; im Vergleich mit Zweikammer-ICD 19\% versus $42 \%, p=0,002)$.

Ähnliche Ergebnisse berichten Chen et al. (2013), die eine signifikant geringere Rate an inappropriaten Schocks in Patienten mit CRT-D beschreiben. Als mögliche Erklärung wird eine Reduktion von Vorhofflimmern, der Hauptursache inappropriater Therapien, durch die kardiale Resynchronisationstherapie genannt. Es wurden nur 185 ICD-Patienten untersucht, davon 85 mit CRT-D. Insgesamt erhielten 18 Patienten einen oder mehrere inappropriate Schocks und die Datenerhebung erfolgte retrospektiv. Zur Verifizierung der Überlegenheit kardialer Resynchronisationstherapie zur Prävention inappropriater Schocks wären weitere prospektive, randomisierte Studien nötig.

Die Programmierung der ICD-Aggregate hat großen Einfluss auf die Erkennung von Tachyarrhythmien und deren korrekte Klassifizierung, was vor allem bei der Verhinderung inappropriater Therapien eine große Rolle spielt. Dabei stellt sich die Frage, ob eine individualisierte, also an den jeweiligen Patienten und dessen Arrhythmieneigung angepasste Programmierung besser ist als eine standardisierte Auswahl basierend auf empirischen Ergebnissen.

Die Daten der Studien EMPIRIC (Wilkoff et al. 2006) und PREPARE (Wilkoff et al. 2008) legen nahe, dass eine empirische ICD-Programmierung mit standardisierter ATP-Aktivierung und einer VT-Zone mit einer Untergrenze von $182 \mathrm{bpm}$ bei mindestens $30 / 40$ Schlägen zu signifikant weniger appropriaten als auch inappropriaten Schocks führt. Bestätigt wird das in der RELEVANT-Studie (Gasparini et al. 2009) bei 
324 Patienten mit nicht-ischämischer Kardiomyopathie, die einen CRT-D zur Primärprophylaxe erhalten hatten.

Moss et al. (2012) beschreiben bei 1500 Patienten der Studie MADIT-RIT (Multicenter Automatic Defibrillator Implantation Trial -Reduce Inappropriate Therapy) die signifikante Reduzierung inappropriater Therapien bei Programmierung einer Therapie ausschließlich für Frequenzen $\geq 200$ bpm mit einer Verzögerung von 2,5 Sekunden gegenüber konventioneller Programmierung, da vor allem atriale Tachyarrhythmien $\leq$ 200 bpm häufig auftreten und mittels ATP inappropriat therapiert werden.

Eine wichtige Beobachtung in den beschriebenen Studien war, dass durch die zunehmend weniger aggressive Programmierung keine Zunahme unbehandelter ventrikulärer Tachykardien aufgetreten ist. Die Anzahl der Synkopen sowie die Mortalitätsraten waren in den jeweiligen Behandlungsgruppen nicht signifikant verschieden. In MADIT-RIT war sogar eine signifikant geringere Mortalität in der Gruppe mit Therapien $\geq 200$ bpm beschrieben worden. Die Zahl der Todesfälle war gering und ein kausaler Zusammenhang mit weniger inappropriaten Therapien kann nicht sicher bestätigt werden (Moss et al. 2012).

Bei den in der vorliegenden Arbeit untersuchten Patienten war die Programmierung über den langen Zeitraum nicht einheitlich. Wie eingangs beschrieben, waren zur Verhinderung inappropriater Therapien aber zahlreiche Detektionsalgorithmen und ATP regelmäßig aktiviert. Die Detektionsgrenzen der VT-Zone waren, insbesondere bei Programmierung einer zusätzlichen FVT-Zone, in der Regel niedriger als in den oben beschriebenen Studien.

Das Ziel muss jeweils sein, das richtige Gleichgewicht zu finden zwischen adäquater Verhinderung inappropriater Therapien bei gleichzeitig sicherer Terminierung lebensbedrohlicher ventrikulärer Tachyarrhythmien.

Aufgrund des retrospektiven Charakters und nicht vollständiger Datenlage ist der Einfluss der Programmierung auf die Häufigkeit von Schocks in der hier untersuchten Population nicht zu bestimmen. Die Ergebnisse von MADIT-RIT, die erst nach Beendigung der Datenaufnahme zu dieser Arbeit vorlagen, haben Eingang in die aktuelle Programmierung gefunden.

Inappropriate ICD-Schocks können zahlreiche negative Auswirkungen haben. Es wurden einzelne Fälle proarrhythmogener Effekte durch inappropriate Schocks beschrieben, die selbst Kammerflimmern auslösten, das zum Tod der Patienten führte (Messali et al. 2004; Veltmann et al. 2007; Catanchin et al. 2008). 
Außerdem wurden Fälle beschrieben, in denen die Entwicklung einer Panikstörung beziehungsweise Agoraphobie bei zwei oder mehr ICD-Schocks pro Jahr signifikant erhöht war (Godemann et al. 2004).

In den großen Studien zur Primär- und Sekundärprophylaxe wurden die Auswirkungen von ICD-Schocks auf die Lebensqualität als eigener Endpunkt untersucht und unterschiedlich bewertet.

Während in der AVID-Studie Schocks einen negativen Einfluss auf die selbst wahrgenommene Lebensqualität hatten (Schron et al. 2002), so war in der ebenfalls sekundärprophylaktischen CIDS-Studie nur bei Auftreten von fünf ICD-Schocks und mehr keine Verbesserung der Lebensqualität aufgetreten (Irvine et al. 2002).

In MADIT-II hingegen verminderten Schocks die Bewertung der gesundheitsbezogenen Lebensqualität am nächsten Untersuchungstermin (Noyes et al. 2009).

Dieser Zusammenhang zwischen dem Abstand von Schocks und der jeweiligen Befragung zur Lebensqualität zeigte sich auch bei den Patienten der SCD-HeFT-Studie. Schocks innerhalb des letzten Monats vor dem jeweiligen Nachbeobachtungstermin waren mit einer verringerten Lebensqualität assoziiert, während dieser Effekt nach einem Jahr nicht mehr festellbar war (Mark et al. 2008).

Es gibt auch Hinweise, dass nicht so sehr Schocks, sondern vielmehr der Fortschritt der Grunderkrankung eine wichtige Rolle spielt. Johansen et al. (2008) zeigen an 610 Patienten, dass eine fortschreitende Herzinsuffizienz in einem höheren Maße mit verringerter Lebensqualität, Ängstlichkeit und depressiver Symptomatik korreliert, als das Auftreten von ICD-Schocks.

Ebenso ist der Einfluss der Schocks selbst oft geringer als das psychologische Profil des Patienten und der Umgang mit der zugrunde liegenden Herzerkrankung. In einer Studie von Pedersen et al. (2004) zeigte sich der Einfluss des Persönlichkeitsprofils auf Angst- und Depressionssymptome stärker als der Einfluss von erlebten Schocks. Depressive Symptome nach einem Myokardinfarkt sind häufig, so waren in einer Untersuchung von Kaptein et al. (2006) etwa ein Viertel der untersuchten 475 Patienten innerhalb des ersten Jahres betroffen.

In der hier beschriebenen Population lag bei insgesamt $42 \%$ der Patienten ein Zustand nach Myokardinfarkt vor. Die Rate von Depressionen betrug lediglich 4\%, da das hier definierte Kriterium einer medikamentösen antidepressiven Therapie zu einer Unterschätzung der wahren Prävalenz geführt haben könnte. Die bewusste Thematisierung dieser Problematik und eventuelle professionelle Hilfe kann den Patienten helfen, mit der individuellen Belastung umzugehen. 


\subsection{Prädiktoren für Tod ohne vorherige appropriate ICD-Therapie}

In der untersuchten Population waren nach $58 \pm 35$ Monaten insgesamt 481 Patienten $(33 \%)$ verstorben, davon $265(20 \%)$ ohne bekannte vorherige appropriate ICDSchocks. 148 dieser 265 Patienten (56\%) hatten den ICD aus primärprophylaktischer Indikation erhalten, was der prozentualen Verteilung in der Gesamtpopulation entspricht.

Der Nutzen einer ICD-Implantation spiegelt sich nicht ausschließlich in appropriater Therapie wider, sondern vor allem in einem Überlebensvorteil. Die Identifikation von Hochrisikopatienten mit hoher Wahrscheinlichkeit eines kurzfristigen nicht arrhythmisch bedingten Todes ist somit ein vorrangiges Ziel bei Indikationsstellung und Implantation.

Unabhängige Prädiktoren für das Ereignis Tod ohne vorherige appropriate ICDTherapie sind eine fortgeschrittene Herzinsuffizienz entsprechend NYHA-Stadium III oder IV (HR: 2,2; 95\% Cl: 1,5 - 3,2; p < 0,001), die Einnahme von Diuretika zum Implantationszeitpunkt (HR: 1,$9 ; 95 \% \mathrm{Cl}: 1,1-3,2 ; \mathrm{p}=0,014$ ) und ein Implantationsalter über 67 Jahren (HR: 2,7; 95\% Cl: 1,9-3,9; $\mathrm{p}<0,001$ ).

Die beiden erstgenannten Parameter haben einen klinisch bedingten Zusammenhang, da bei schweren Formen der Herzinsuffizienz eine Diuretikatherapie häufig ist. Auch wenn es bisher keine Daten zur Mortalitätsreduktion durch Diuretika bei Herzinsuffizienz gibt, ist nach den aktuellen Leitlinien der Europäischen Gesellschaft für Kardiologie die Gabe von Diuretika bei Zeichen von Herzinsuffizienz, wie Dyspnoe und Flüssigkeitsretention, empfohlen (McMurray et al. 2012).

Bei den in der vorliegenden Arbeit untersuchten Patienten war die Anzahl appropriater Therapien unabhängig von der Diuretikaeinnahme (HR: 1,063; 95\% Cl: 0,8 - 1,4; $p=0,646)$, das Risiko für appropriate Therapien aber verdoppelt.

Dieser Zusammenhang zeigte sich auch bei Koller et al. (2008). Eine DiuretikaEinnahme war sogar mit einem 4-fach erhöhten Risiko assoziiert. In der prospektiven Untersuchung von 422 Patienten waren insgesamt 73 Patienten nach einem mittleren Follow-Up von 3,6 Jahren verstorben, davon 29 Patienten, die keine appropriate Therapie erhalten hatten. Ein hohes Alter zeigte eine Tendenz für Tod ohne vorherige appropriate Therapie, erreichte aber keine statistische Signifikanz, am wahrscheinlichsten aufgrund der geringen Fallzahl. 
Die Bewertung, ob eine ICD-Implantation bei Patienten in hohem Alter und mit fortgeschrittener Herzinsuffizienz von Nutzen ist, wird kontrovers diskutiert.

In einer Studie mit 1866 Patienten, davon 252 im Alter $\geq 75$ Jahren, zeigte sich ein signifikant höheres Risiko für nichtarrhythmisch bedingten Tod (8,7\% pro Jahr versus $4 \%$ pro Jahr) und daher ein eventuell geringerer Nutzen einer ICD-Implantation bei Älteren (Healey et al. 2007).

In einer Metaanalyse von 11 Studien wurde gezeigt, dass die jährliche Mortalitätsrate mit dem Grad der Herzinsuffizienz steigt, von 15\% bei NYHA-Stadium I und II auf bis zu 60\% bei NYHA-Stadium IV. Dabei sinkt aber der Anteil des plötzlichen, arrhythmiebedingten Herztods von $50-60 \%$ bei NYHA-Stadium I und II auf $20-30 \%$ bei NYHA-Stadium IV. Die Haupttodesursache bei hochgradiger Herzinsuffizienz ist progressives Pumpversagen, während ein plötzlicher Herztod eher bei leichtgradig symptomatischen oder asymptomatischen Patienten auftritt. Eine mögliche Ursache kann der Verlust myokardialer Katecholamine und einer verminderten Dichte der $\beta$ Adrenozeptoren des Myokards sein, die zu einer erhöhten Toleranz für ventrikuläre Tachyarrhythmien führt (Kjekshus 1990).

Es gibt aber auch Hinweise, dass vor allem ältere Patienten mit fortgeschrittener Herzinsuffizienz von einer ICD-Implantation profitieren.

So entwickelten Sheldon et al. (2000) bei 659 untersuchten Patienten der CIDSStudie einen Risiko-Score. Der größte Überlebensvorteil zeigte sich bei Vorliegen von zwei der folgenden drei klinischen Parameter: Alter > 70 Jahre, NYHA-Stadium III oder IV und eine LVEF < 35\%.

Goldenberg et al. (2008) zeigten an 1232 Patienten der MADIT-II-Studie einen signifikanten Nutzen einer ICD-Implantation, wenn mindestens einer der folgenden Risikofaktoren besteht: Alter $>70$ Jahre, NYHA-Stadium > II, eingeschränkte Nierenfunktion, QRS-Breite $>120$ ms oder Vorhofflimmern. Kein Überlebensvorteil zeigte sich hingegen in der Hochrisikogruppe von Patienten mit schwerer Niereninsuffizienz (Serumkreatinin $\geq 2,5 \mathrm{mg} / \mathrm{dl}$ ).

In einer Untersuchung von 1089 Patienten mit Herzinsuffizienz im Endstadium, die für eine Herztransplantation gelistet waren, zeigte sich in der Gruppe mit 550 ICDPatienten eine deutliche Reduktion der Gesamtmortalität. Am größten war diese mit $60 \%$ bei Patienten mit primärprophylaktischer Implantation (Fröhlich et al. 2013).

Ein wichtiger Punkt in diesem Zusammenhang ist auch die Mortalitätsreduktion durch kardiale Resynchronisationstherapie bei Patienten mit hochgradiger Herzinsuffizienz. 
Neben der bereits erwähnten Mortalitätsreduktion von $36 \%$ in der COMPANIONStudie (Bristow et al. 2004) zeigte sich bei 813 Patienten der CARE-HF-Studie (Cardiac Resynchronization - Heart Failure) mit LVEF $\leq 35 \%$ und überwiegend Symptomen entsprechend NYHA-Stadium III sowohl eine 45-prozentige Reduktion von Todesfällen durch Pumpversagen als auch eine Reduktion des plötzlichen Herztodes von 46\% (Cleland et al. 2006).

Insgesamt wurde in der hier untersuchten Population von 1484 Patienten bei 31\% ein CRT-D implantiert. Von den 265 ohne vorherige appropriate Therapie Verstorbenen hatte ein prozentual größerer Anteil (103 Patienten, 39\%) ein Gerät zur kardialen Resynchronisationstherapie erhalten.

Eine Analyse von Prädiktoren für Tod ohne vorherige appropriate ICD-Therapie führten auch van Rees et al. (2012) durch und kamen dabei zu ähnlichen Ergebnissen wie in der vorliegenden Arbeit. Untersucht wurden 900 Patienten mit ischämischer Kardiomyopathie, die einen ICD zur Primärprophylaxe erhalten hatten. 191 Patienten (21\%) erhielten appropriate Therapien, 150 (17\%) sind im medianen Nachbeobachtungszeitraum von 669 Tagen verstorben, 114 Patienten (13\% aller Patienten beziehungsweise $76 \%$ aller Verstorbenen) ohne vorherige appropriate Therapie. Unabhängige Prädiktoren dafür waren NYHA-Stadium $\geq$ III, höheres Alter, Diabetes mellitus, LVEF $\leq 25 \%$ und Nikotinkonsum.

Bei der Indikationsstellung sollte aber nicht das Lebensalter der Patienten an sich eine Rolle spielen, sondern vielmehr die individuelle Evaluation der Lebenserwartung. Dass dies bereits geschieht und trotzdem im Einzelfall schwierig sein kann, ist aufgrund der oft komplexen Lebens- und Krankengeschichten der Patienten verständlich.

Eine ebenfalls wichtige Thematik ist der Umgang mit nötig werdenden Aggregatwechseln bei diesen Patienten. In der untersuchten Population hatten bei insgesamt 326 Fällen mit Batterieerschöpfung 204 Patienten (63\%) keine vorherige appropriate Therapie durch den ICD erhalten.

Koller et al. (2008) geben die Wahrscheinlichkeit für erstmals auftretende arrhythmische Ereignisse 6-8 Jahre nach Implantation mit 6\% an, das Neuauftreten von Kammerflimmern sogar als höchst unwahrscheinlich. 
Grimm und Marchlinski (1994) beschreiben, dass von 26 Patienten ohne vorherige Schocks bis zur Batterieerschöpfung bei 35\% im Verlauf appropriate Schocks auftreten, teilweise mehr als 7 Jahre nach Erstimplantation.

Eine generelle Schlussfolgerung lässt sich daher nicht ziehen, es muss eine sorgfältige Abwägung erfolgen zwischen einer potentiell lebensrettenden Therapie und den eventuellen Einschränkungen der Lebensqualität durch eben diese Therapie.

\subsection{Limitationen}

Die vorliegende Arbeit ist eine retrospektive Untersuchung und daher nicht vergleichbar mit prospektiv durchgeführten, randomisierten Studien. In diesem Zusammenhang müssen die Ergebnisse der durchgeführten Analysen von Prädiktoren gesehen werden.

In dem großen Zeitraum von fast 13 Jahren, in dem Implantationen vorgenommen wurden, gab es fortlaufende Veränderungen in Indikationsstellung, wie auch Fortschritte in Geräteentwicklung und Programmierung sowie Neuentwicklungen in der Pharmakotherapie. Dadurch ist eine heterogene Population entstanden, die mit randomisierten klinischen Studien nicht unbedingt vergleichbar ist, die oft zahlreiche Einund Ausschlusskriterien haben. Andererseits spiegelt die untersuchte Population die klinische Routine wider und kann insofern auch praktikable Ergebnisse liefern. Die hier beschriebenen Patienten haben sich ausschließlich in einem Zentrum der ICDImplantation unterzogen. Daher ist es möglich, dass die Praxis der Indikationsstellung und Programmierung sowie die demographischen und klinischen Parameter in anderen Zentren abweichen.

Außerdem konnte in dieser Analyse der Einfluss der einzelnen Parameter der ICDProgrammierung nicht einbezogen werden.

Die Auswertung und Klassifizierung der Therapieepisoden erfolgte nur durch einen einzelnen Kardiologen und wurde nicht durch eine zweite Instanz überprüft. Eine vereinzelte falsche Bewertung appropriater und inappropriater Therapien ist daher nicht komplett auszuschließen.

Trotz der Versuche, aus allen verfügbaren Quellen Informationen über Art und Häufigkeit der aufgetretenen ICD-Therapien zu erhalten, könnten nicht alle potentiell aufgetretenen Schocks registriert worden sein, vor allem, da kein postmortales Auslesen der Geräte erfolgte. Dies ist vor allem entscheidend bei der Auswertung bezüglich des Todes ohne vorherige appropriate Therapie. 
Auch nach gewissenhafter Auswahl der klinisch relevanten Faktoren für die Analyse von Prädiktoren ist es denkbar, dass es weitere Parameter gab, die einen Einfluss auf den jeweiligen Endpunkt hatten, aber in der vorliegenden Arbeit nicht untersucht wurden. Außerdem wurden nur Kriterien zum Zeitpunkt der Implantation betrachtet, Ereignisse in der Nachbeobachtungszeit wie zwischenzeitliche Verschlechterung einer Herzinsuffizienz, myokardiale Ischämien oder Wechsel der Medikation wurden hingegen nicht einbezogen.

\subsection{Ausblick}

Es besteht weiterhin das Problem, dass ein nicht zu vernachlässigender Teil der $\mathrm{Pa}$ tienten, die einen plötzlichen Herztod erleiden, nicht die klassischen Implantationskriterien für einen ICD erfüllen. Dies gilt vor allem, wenn der plötzliche Herztod die erste Manifestation einer kardialen Erkrankung ist. Um diese Patienten besser identifizieren zu können, sind neben der klassischen epidemiologischen Analyse klinischer Prädiktoren und Risikofaktoren auch weitere, neue Ansätze nötig. Vielversprechend in dieser Hinsicht sind Untersuchungen, inwieweit genetische Einflüsse bei der Entstehung von ventrikulären Tachyarrhythmien und der Begünstigung eines plötzlichen Herztodes eine Rolle spielen. Dies können Polymorphismen in Genen sein, die in Verbindung stehen mit der kardialen Erregungsleitung (Kanalopathien) oder dem sympathischen (adrenergen) Nervensystem (Deo und Albert 2012).

In einer aktuell laufenden prospektiven europäischen Multizenter-Studie wird ein Einfluss 19 solcher Gene auf ein erhöhtes arrhythmogenes Potential untersucht (Seegers et al. 2012). Daneben wird auch der Einfluss verschiedener elektrophysiologischer Parameter wie T-Wellen-Morphologie und T-Wellen-Alternans und der Herzfrequenzvariabilität untersucht.

Die immer genauer werdende Eingrenzung, welche Patienten das höchste Risiko für einen plötzlichen Herztod und daher den größten Nutzen der Implantation eines ICD haben, wird voraussichtlich durch diese neuen Herangehensweisen weiter fortschreiten, um im Endeffekt nicht nur den Patienten zu dienen, sondern auch denen, die keinen ICD benötigen. 


\section{Zusammenfassung}

Der plötzliche Herztod, eine der häufigsten Todesursachen, ist meist tachyarrhythmisch bedingt und kann wirksam durch einen implantierbaren KardioverterDefibrillator (ICD) verhindert werden. Dafür ist eine möglichst genaue Auswahl der Patienten, die ein erhöhtes Risiko für einen plötzlichen Herztod haben, nötig.

Die vorliegende Arbeit untersuchte das Vorhandensein von Prädiktoren für das Auftreten von appropriaten und inappropriaten Schocks sowie Tod ohne vorherige appropriate Therapie bei 1484 ICD-Patienten. Als unabhängige Prädiktoren für das Auftreten von appropriaten ICD-Schocks zeigten sich ein männliches Geschlecht, eine Amiodaroneinnahme zum Zeitpunkt der Implantation und eine sekundärprophylaktische Implantationsindikation. Vorhofflimmern und ein Implantationsalter unter 67 Jahren waren mit einem häufigeren Auftreten von inappropriaten Schocks assoziiert. Das Risiko, ohne vorherige appropriate Therapie des ICD zu versterben, war bei $\mathrm{Pa}$ tienten mit fortgeschrittener Herzinsuffizienz, entsprechend NYHA-Stadium III / IV, der Einnahme von Diuretika und einem Implantationsalter über 67 Jahren erhöht. Die Prädiktoranalyse ist aus mehreren Gründen relevant. Es gilt, die Patienten mit erhöhtem Risiko für ventrikuläre Tachyarrhythmien zu identifizieren, um die Therapie zu optimieren und eventuell zu intensivieren. Da es sich bei inappropriaten Schocks um die häufigste Nebenwirkung der ICD-Therapie handelt, die zudem noch mit deutlichen Einschränkungen bezüglich Lebensqualität und Akzeptanz der Therapie einhergehen kann, ist es wichtig, bei Risikopatienten gegebenenfalls Strategien für Prävention und Umgang mit inappropriaten Schocks zu entwickeln. Trotz sorgfältiger Indikationsstellung zeigte sich, dass ein nicht unerheblicher Teil der untersuchten Patientenpopulation ohne vorherige appropriate ICD-Therapie verstorben ist. Diese Patienten, die von einer Implantation nicht profitieren, noch genauer zu klassifizieren, hilft nicht nur, kosteneffektiv die vorhandenen Möglichkeiten optimal auszuschöpfen, sondern auch, eventuelle Komplikationen der Therapie von vornherein zu vermeiden. Da dies nicht einfach zu lösen ist, weil die genannten Patienten ein sowohl hohes Risiko für arrhythmisch bedingten plötzlichen Herztod haben als auch eine hohe Mortalitätsrate aus konkurrierenden Gründen, soll noch einmal betont werden, wie wichtig ein möglichst bedarfsgerechter Einsatz dieser so oft lebensrettenden Therapie durch den ICD ist. 


\section{Literaturverzeichnis}

Albert CM, Chae CU, Grodstein F, Rose LM, Rexrode KM, Ruskin JN, Stampfer MJ, Manson JE (2003): Prospective study of sudden cardiac death among women in the United States. Circulation 107, 2096-2101

Alter P, Waldhans S, Plachta E, Moosdorf R, Grimm W (2005): Complications of Implantable Cardioverter Defibrillator Therapy in 440 Consecutive Patients. Pacing Clin Electrophysiol 28, 926-932

Bardy GH, Lee KL, Mark DB, Poole JE, Packer DL, Boineau R, Domanski M, Troutman C, Anderson J, Johnson G et al. (2005): Amiodarone or an implantable cardioverter-defibrillator for congestive heart failure. N Engl J Med 352, 225-237

Bays HE (2011): Adiposopathy is "sick fat" a cardiovascular disease? J Am Coll Cardiol $\underline{57}, 2461-2473$

Bhavnani SP, Coleman CI, White CM, Clyne CA, Yarlagadda R, Guertin D, Kluger J (2008): Association between statin therapy and reductions in atrial fibrillation or flutter and inappropriate shock therapy. Europace $\underline{10}$, 854-859

Bhavnani SP, Pavuluri V, Coleman Cl, Guertin D, Yarlagadda RK, Clyne CA, Kluger J (2013): The Gender-Paradox among Patients with Implantable CardioverterDefibrillators: A Propensity-Matched Study. Pacing Clin Electrophysiol $\underline{36}$, 878-884

Bristow MR, Saxon LA, Boehmer J, Krueger S, Kass DA, Marco T de, Carson P, DiCarlo L, DeMets D, White BG et al. (2004): Cardiac-resynchronization therapy with or without an implantable defibrillator in advanced chronic heart failure. $\mathrm{N}$ Engl J Med $\underline{350}, 2140-2150$

Buxton AE, Lee KL, Fisher JD, Josephson ME, Prystowsky EN, Hafley G (1999): A randomized study of the prevention of sudden death in patients with coronary artery disease. Multicenter Unsustained Tachycardia Trial Investigators. N Engl J Med $\underline{341}$, 1882-1890

Catanchin A, Anderson L, Jones SU, Ward D (2008): When Life-Saving Devices Terminate Life. J Cardiovasc Electrophysiol 19, 316-318

Chen Z, Kotecha T, Crichton S, Shetty A, Sohal M, Arujuna A, Kirubakaran S, Bostock J, Cooklin M, O'Neill M et al. (2013): Lower incidence of inappropriate shock therapy in patients with combined cardiac resynchronisation therapy defibrillators 
(CRT-D) compared with patients with non-CRT defibrillators (ICDs). Int J Clin Pract $\underline{67}, 733-739$

Cleland JGF, Daubert J, Erdmann E, Freemantle N, Gras D, Kappenberger L, Tavazzi $L$ (2006): Longer-term effects of cardiac resynchronization therapy on mortality in heart failure the CArdiac REsynchronization-Heart Failure (CARE-HF) trial extension phase. Eur Heart J $\underline{27}, 1928-1932$

Connolly SJ (1999): Evidence-based analysis of amiodarone efficacy and safety. Circulation $\underline{100}, 2025-2034$

Connolly SJ, Gent M, Roberts RS, Dorian P, Roy D, Sheldon RS, Mitchell LB, Green MS, Klein GJ, O'Brien B (2000): Canadian implantable defibrillator study (CIDS): A randomized trial of the implantable cardioverter defibrillator against amiodarone. Circulation $\underline{101}, 1297-1302$

Connolly SJ, Dorian P, Roberts RS, Gent M, Bailin S, Fain ES, Thorpe K, Champagne J, Talajic M, Coutu B et al. (2006): Comparison of beta-blockers, amiodarone plus beta-blockers, or sotalol for prevention of shocks from implantable cardioverter defibrillators: the OPTIC Study: a randomized trial. JAMA $\underline{295}, 165-171$

Daubert JP, Zareba W, Cannom DS, McNitt S, Rosero SZ, Wang P, Schuger C, Steinberg JS, Higgins SL, Wilber DJ et al. (2008): Inappropriate implantable cardioverter-defibrillator shocks in MADIT II: frequency, mechanisms, predictors, and

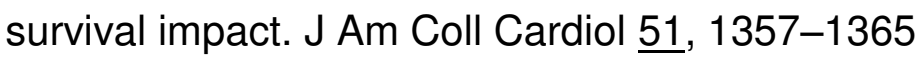

Deisenhofer I, Kolb C, Ndrepepa G, Schreieck J, Karch M, Schmieder S, Zrenner B, Schmitt C (2001): Do Current Dual Chamber Cardioverter Defibrillators Have Advantages Over Conventional Single Chamber Cardioverter Defibrillators in Reducing Inappropriate Therapies? A Randomized, Prospective Study. J Cardiovasc Electrophysiol 12, 134-142

Deo R, Albert CM (2012): Epidemiology and genetics of sudden cardiac death. Circulation $\underline{125}, 620-637$

Dickstein K, Vardas PE, Auricchio A, Daubert J, Linde C, McMurray J, Ponikowski P, Priori SG, Sutton R, van Veldhuisen DJ (2010): 2010 focused update of ESC Guidelines on device therapy in heart failure: an update of the 2008 ESC Guidelines for the diagnosis and treatment of acute and chronic heart failure and the 2007 ESC Guidelines for cardiac and resynchronization therapy. Developed with the special contribu- 
tion of the Heart Failure Association and the European Heart Rhythm Association. Eur J Heart Fail 12, 1143-1153

Epstein AE, DiMarco JP, Ellenbogen KA, Estes NAM III, Freedman RA, Gettes LS, Gillinov AM, Gregoratos G, Hammill SC, Hayes DL et al. (2008): ACC/AHA/HRS 2008 Guidelines for Device-Based Therapy of Cardiac Rhythm Abnormalities: a report of the American College of Cardiology/American Heart Association Task Force on Practice Guidelines (Writing Committee to Revise the ACC/AHA/NASPE 2002 Guideline Update for Implantation of Cardiac Pacemakers and Antiarrhythmia Devices) developed in collaboration with the American Association for Thoracic Surgery and Society of Thoracic Surgeons. J Am Coll Cardiol 51, e1-62

Epstein AE, DiMarco JP, Ellenbogen KA, Estes NAM, Freedman RA, Gettes LS, Gillinov AM, Gregoratos G, Hammill SC, Hayes DL et al. (2013): 2012 ACCF/AHA/HRS focused update incorporated into the ACCF/AHA/HRS 2008 guidelines for device-based therapy of cardiac rhythm abnormalities: a report of the American College of Cardiology Foundation/American Heart Association Task Force on Practice Guidelines and the Heart Rhythm Society. J Am Coll Cardiol 61, e6-75

Exner DV, Pinski SL, Wyse DG, Renfroe EG, Follmann D, Gold M, Beckman KJ, Coromilas J, Lancaster S, Hallstrom AP (2001): Electrical storm presages nonsudden death: the antiarrhythmics versus implantable defibrillators (AVID) trial. Circulation $\underline{103}, 2066-2071$

Friedman PA, McClelland RL, Bamlet WR, Acosta H, Kessler D, Munger TM, Kavesh NG, Wood M, Daoud E, Massumi A et al. (2006): Dual-chamber versus singlechamber detection enhancements for implantable defibrillator rhythm diagnosis: the detect supraventricular tachycardia study. Circulation $\underline{113}, 2871-2879$

Fröhlich GM, Holzmeister J, Hubler M, Hubler S, Wolfrum M, Enseleit F, Seifert B, Hurlimann D, Lehmkuhl HB, Noll G et al. (2013): Prophylactic implantable cardioverter defibrillator treatment in patients with end-stage heart failure awaiting heart transplantation. Heart $\underline{99}, 1158-1165$

Gandjour A, Holler A, Dipl-Ges-Ok, Adarkwah CC (2011): Cost-effectiveness of implantable defibrillators after myocardial infarction based on 8-year follow-up data (MADIT II). Value Health $\underline{14}, 812-817$

Gasparini M, Menozzi C, Proclemer A, Landolina M, lacopino S, Carboni A, Lombardo E, Regoli F, Biffi M, Burrone V et al. (2009): A simplified biventricular defibrillator 
with fixed long detection intervals reduces implantable cardioverter defibrillator (ICD) interventions and heart failure hospitalizations in patients with non-ischaemic cardiomyopathy implanted for primary prevention: the RELEVANT [Role of long dEtection window programming in patients with LEft VentriculAr dysfunction, Non-ischemic eTiology in primary prevention treated with a biventricular ICD] study. European Heart Journal $\underline{30}$, 2758-2767

Ghanbari H, Dalloul G, Hasan R, Daccarett M, Saba S, David S, Machado C (2009): Effectiveness of implantable cardioverter-defibrillators for the primary prevention of sudden cardiac death in women with advanced heart failure: a meta-analysis of randomized controlled trials. Arch Intern Med 169, 1500-1506

Godemann F, Butter C, Lampe F, Linden M, Schlegl M, Schultheiss H, Behrens S (2004): Panic disorders and agoraphobia: side effects of treatment with an implantable cardioverter/defibrillator. Clin Cardiol $\underline{27}, 321-326$

Goldenberg I, Moss AJ, Maron BJ, Dick AW, Zareba W (2005): Cost-effectiveness of implanted defibrillators in young people with inherited cardiac arrhythmias. Ann Noninvasive Electrocardiol 10, 67-83

Goldenberg I, Vyas AK, Hall WJ, Moss AJ, Wang H, He H, Zareba W, McNitt S, Andrews ML (2008): Risk stratification for primary implantation of a cardioverterdefibrillator in patients with ischemic left ventricular dysfunction. J Am Coll Cardiol $\underline{51}$, 288-296

Greene HL (1993): The CASCADE Study: randomized antiarrhythmic drug therapy in survivors of cardiac arrest in Seattle. CASCADE Investigators. Am J Cardiol $\underline{72}, 70 \mathrm{~F}-$ $74 \mathrm{~F}$

Grimm W, Marchlinski FE (1994): Shock occurrence in patients with an implantable cardioverter-defibrillator without spontaneous shocks before first generator replacement for battery depletion. Am J Cardiol $\underline{73}, 969-970$

Grönefeld GC, Mauss O, Li YG, Klingenheben T, Hohnloser SH (2000): Association between atrial fibrillation and appropriate implantable cardioverter defibrillator therapy: results from a prospective study. J Cardiovasc Electrophysiol 11, 1208-1214

Haigney MC, Zareba W, Nasir JM, McNitt S, McAdams D, Gentlesk PJ, Goldstein RE, Moss AJ (2009): Gender differences and risk of ventricular tachycardia or ventricular fibrillation. Heart Rhythm $\underline{6}, 180-186$ 
Healey JS, Hallstrom AP, Kuck K, Nair G, Schron EP, Roberts RS, Morillo CA, Connolly SJ (2007): Role of the implantable defibrillator among elderly patients with a history of life-threatening ventricular arrhythmias. Eur Heart J $\underline{28}, 1746-1749$

Hohnloser SH, Kuck KH, Dorian P, Roberts RS, Hampton JR, Hatala R, Fain E, Gent M, Connolly SJ (2004): Prophylactic use of an implantable cardioverter-defibrillator after acute myocardial infarction. N Engl J Med $\underline{351}, 2481-2488$

Hohnloser SH, Dorian P, Roberts R, Gent M, Israel CW, Fain E, Champagne J, Connolly SJ (2006): Effect of amiodarone and sotalol on ventricular defibrillation threshold: the optimal pharmacological therapy in cardioverter defibrillator patients (OPTIC) trial. Circulation 114, 104-109

Irvine J, Dorian P, Baker B, O'Brien BJ, Roberts R, Gent M, Newman D, Connolly SJ (2002): Quality of life in the Canadian Implantable Defibrillator Study (CIDS). Am Heart J $\underline{144}, 282-289$

Johansen JB, Pedersen SS, Spindler H, Andersen K, Nielsen JC, Mortensen PT (2008): Symptomatic heart failure is the most important clinical correlate of impaired quality of life, anxiety, and depression in implantable cardioverter-defibrillator patients: a single-centre, cross-sectional study in 610 patients. Europace $\underline{10}, 545-551$

Josephson M, Wellens HJJ (2004): Implantable Defibrillators and Sudden Cardiac Death. Circulation $\underline{109}, 2685-2691$

Kadish A, Dyer A, Daubert JP, Quigg R, Estes NAM III, Anderson KP, Calkins H, Hoch D, Goldberger J, Shalaby A et al. (2004): Prophylactic defibrillator implantation in patients with nonischemic dilated cardiomyopathy. N Engl J Med $\underline{350}, 2151-2158$

Kannel WB, Wolf PA, Benjamin EJ, Levy D (1998): Prevalence, incidence, prognosis, and predisposing conditions for atrial fibrillation: population-based estimates. Am J Cardiol $\underline{82}, 2 \mathrm{~N}-9 \mathrm{~N}$

Kaptein KI, Jonge P de, van den Brink RHS, Korf J (2006): Course of depressive symptoms after myocardial infarction and cardiac prognosis: a latent class analysis. Psychosom Med $\underline{68}, 662-668$

Kjekshus J (1990): Arrhythmias and mortality in congestive heart failure. Am J Cardiol $\underline{65}, 42 \mathrm{l}-48 \mathrm{I}$

Kleemann T, Becker T, Doenges K, Vater M, Senges J, Schneider S, Saggau W, Weisse U, Seidl K (2007): Annual rate of transvenous defibrillation lead defects in 
implantable cardioverter-defibrillators over a period of 10 years. Circulation $\underline{115}$, 2474-2480

Klein G, Lissel C, Fuchs A, Gardiwal A, Oswald H, Desousa M, Pichlmaier AM, Lichtinghagen R, Geerlings H, Lippolt P et al. (2006): Predictors of VT/VF-occurrence in ICD patients: results from the PROFIT-Study. Europace $\underline{8}, 618-624$

Klein RC, Raitt MH, Wilkoff BL, Beckman KJ, Coromilas J, Wyse DG, Friedman PL, Martins JB, Epstein AE, Hallstrom AP et al. (2003): Analysis of implantable cardioverter defibrillator therapy in the Antiarrhythmics Versus Implantable Defibrillators (AVID) Trial. J Cardiovasc Electrophysiol 14, 940-948

Koller MT, Schaer B, Wolbers M, Sticherling C, Bucher HC, Osswald S (2008): Death without prior appropriate implantable cardioverter-defibrillator therapy: a competing risk study. Circulation $\underline{117}, 1918-1926$

Kuck KH, Cappato R, Siebels J, Ruppel R (2000): Randomized comparison of antiarrhythmic drug therapy with implantable defibrillators in patients resuscitated from cardiac arrest: The Cardiac Arrest Study Hamburg (CASH). Circulation 102, 748-754 MacFadden DR, Crystal E, Krahn AD, Mangat I, Healey JS, Dorian P, Birnie D, Simpson CS, Khaykin Y, Pinter A et al. (2012): Sex differences in implantable cardioverter-defibrillator outcomes: findings from a prospective defibrillator database. Ann Intern Med 156, 195-203

Mark DB, Anstrom KJ, Sun JL, Clapp-Channing NE, Tsiatis AA, Davidson-Ray L, Lee KL, Bardy GH (2008): Quality of life with defibrillator therapy or amiodarone in heart failure. N Engl J Med 359, 999-1008

McMurray JJV, Adamopoulos S, Anker SD, Auricchio A, Bohm M, Dickstein K, Falk V, Filippatos G, Fonseca C, Gomez-Sanchez MA et al. (2012): ESC guidelines for the diagnosis and treatment of acute and chronic heart failure 2012: The Task Force for the Diagnosis and Treatment of Acute and Chronic Heart Failure 2012 of the European Society of Cardiology. Developed in collaboration with the Heart Failure Association (HFA) of the ESC. Eur J Heart Fail 14, 803-869

Messali A, Thomas O, Chauvin M, Coumel P, Leenhardt A (2004): Death due to an implantable cardioverter defibrillator. J Cardiovasc Electrophysiol 15, 953-956 
Mikkola TS, Gissler M, Merikukka M, Tuomikoski P, Ylikorkala O, Onland-Moret NC (2013): Sex Differences in Age-Related Cardiovascular Mortality. PLoS ONE $\underline{8}$, e63347

Moss AJ, Hall WJ, Cannom DS, Daubert JP, Higgins SL, Klein H, Levine JH, Saksena S, Waldo AL, Wilber D et al. (1996): Improved survival with an implanted defibrillator in patients with coronary disease at high risk for ventricular arrhythmia. Multicenter Automatic Defibrillator Implantation Trial Investigators. N Engl J Med $\underline{335}$, 1933-1940

Moss AJ, Zareba W, Hall WJ, Klein H, Wilber DJ, Cannom DS, Daubert JP, Higgins SL, Brown MW, Andrews ML (2002): Prophylactic implantation of a defibrillator in patients with myocardial infarction and reduced ejection fraction. N Engl J Med $\underline{346}$, 877-883

Moss AJ, Hall WJ, Cannom DS, Klein H, Brown MW, Daubert JP, Estes NAM III, Foster E, Greenberg H, Higgins SL et al. (2009): Cardiac-resynchronization therapy for the prevention of heart-failure events. N Engl J Med $\underline{361}, 1329-1338$

Moss AJ, Schuger C, Beck CA, Brown MW, Cannom DS, Daubert JP, Estes NAM III, Greenberg H, Hall WJ, Huang DT et al. (2012): Reduction in Inappropriate Therapy and Mortality through ICD Programming. N Engl J Med $\underline{367}, 2275-2283$

Nichol G, Thomas E, Callaway CW, Hedges J, Powell JL, Aufderheide TP, Rea T, Lowe R, Brown T, Dreyer J et al. (2008): Regional variation in out-of-hospital cardiac arrest incidence and outcome. JAMA $\underline{300}, 1423-1431$

Noyes K, Corona E, Veazie P, Dick AW, Zhao H, Moss AJ (2009): Examination of the effect of implantable cardioverter-defibrillators on health-related quality of life: based on results from the Multicenter Automatic Defibrillator Trial-II. Am J Cardiovasc Drugs $\underline{9}, 393-400$

Pedersen SS, van Domburg RT, Theuns DAMJ, Jordaens L, Erdman RAM (2004): Type D personality is associated with increased anxiety and depressive symptoms in patients with an implantable cardioverter defibrillator and their partners. Psychosom Med $\underline{66}, 714-719$

Ruwald AH, Sood N, Ruwald MH, Jons C, Clyne CA, McNitt S, Wang P, Zareba W, Moss AJ (2013): Frequency of inappropriate therapy in patients implanted with dualversus single-chamber ICD devices in the ICD arm of MADIT-CRT. J Cardiovasc Electrophysiol 24, 672-679 
Santangeli P, Pelargonio G, Dello Russo A, Casella M, Bisceglia C, Bartoletti S, Santarelli P, Di Biase L, Natale A (2010): Gender differences in clinical outcome and primary prevention defibrillator benefit in patients with severe left ventricular dysfunction: a systematic review and meta-analysis. Heart Rhythm $\underline{7}, 876-882$

Schoels W, Steinhaus D, Johnson WB, O'hara G, Schwab JO, Jenniskens I, DeGroot PJ, Tang F, Helmling E (2007): Optimizing implantable cardioverter-defibrillator treatment of rapid ventricular tachycardia: antitachycardia pacing therapy during charging. Heart Rhythm 4, $879-885$

Schron EB, Exner DV, Yao Q, Jenkins LS, Steinberg JS, Cook JR, Kutalek SP, Friedman PL, Bubien RS, Page RL et al. (2002): Quality of life in the antiarrhythmics versus implantable defibrillators trial: impact of therapy and influence of adverse symptoms and defibrillator shocks. Circulation $\underline{105}, 589-594$

Seegers J, Vos MA, Flevari P, Willems R, Sohns C, Vollmann D, Luthje L, Kremastinos DT, Flore V, Meine M et al. (2012): Rationale, objectives, and design of the EUTrigTreat clinical study: a prospective observational study for arrhythmia risk stratification and assessment of interrelationships among repolarization markers and genotype. Europace 14, 416-422

Sheldon R, Connolly S, Krahn A, Roberts R, Gent M, Gardner M (2000): Identification of patients most likely to benefit from implantable cardioverter-defibrillator therapy: the Canadian Implantable Defibrillator Study. Circulation 101, 1660-1664

Smith T, Jordaens L, Theuns DAMJ, van Dessel PF, Wilde AA, Hunink MGM (2013): The cost-effectiveness of primary prophylactic implantable defibrillator therapy in patients with ischaemic or non-ischaemic heart disease: a European analysis. Eur Heart J $\underline{34}, 211-219$

Soundarraj D, Thakur RK, Gardiner JC, Khasnis A, Jongnarangsin K (2006): Inappropriate ICD Therapy: Does Device Configuration Make a Difference. Pacing Clin Electrophysiol $\underline{29}, 810-815$

Stein KM, Euler DE, Mehra R, Seidl K, Slotwiner DJ, Mittal S, Markowitz SM, Lerman BB (2002): Do atrial tachyarrhythmias beget ventricular tachyarrhythmias in defibrillator recipients? J Am Coll Cardiol 40, 335-340

The Antiarrhythmics versus Implantable Defibrillators (AVID) Investigators (1997): A comparison of antiarrhythmic-drug therapy with implantable defibrillators in patients resuscitated from near-fatal ventricular arrhythmias. N Engl J Med 337, 1576-1583 
Theuns DAMJ, Klootwijk APJ, Goedhart DM, Jordaens LJLM (2004): Prevention of inappropriate therapy in implantable cardioverter-defibrillators: results of a prospective, randomized study of tachyarrhythmia detection algorithms. J Am Coll Cardiol $\underline{44}$, 2362-2367

Tompkins C, McNitt S, Daubert J, Wang P, Moss A, Zareba W (2012): Gender differences in inappropriate device therapies from MADIT. J Am Coll Cardiol $\underline{59}$, E583

Trappe H (2012): Plötzlicher Herztod und automatisierte externe Defibrillatoren. Herz $\underline{37}, 416-423$

van Rees JB, Borleffs CJW, Bie MK de, Stijnen T, van Erven L, Bax JJ, Schalij MJ (2011): Inappropriate implantable cardioverter-defibrillator shocks: incidence, predictors, and impact on mortality. J Am Coll Cardiol 57, 556-562

van Rees JB, Borleffs CJW, van Welsenes GH, van der Velde ET, Bax JJ, van Erven L, Putter H, van der Bom JG, Schalij MJ (2012): Clinical prediction model for death prior to appropriate therapy in primary prevention implantable cardioverter defibrillator patients with ischaemic heart disease: the FADES risk score. Heart $\underline{98}, 872-877$

Veltmann C, Borggrefe M, Schimpf R, Wolpert C (2007): Fatal inappropriate ICD shock. J Cardiovasc Electrophysiol 18, 326-328

Wathen MS, Sweeney MO, DeGroot PJ, Stark AJ, Koehler JL, Chisner MB, Machado C, Adkisson WO (2001): Shock reduction using antitachycardia pacing for spontaneous rapid ventricular tachycardia in patients with coronary artery disease. Circulation 104, 796-801

Wathen MS, DeGroot PJ, Sweeney MO, Stark AJ, Otterness MF, Adkisson WO, Canby RC, Khalighi K, Machado C, Rubenstein DS et al. (2004): Prospective randomized multicenter trial of empirical antitachycardia pacing versus shocks for spontaneous rapid ventricular tachycardia in patients with implantable cardioverterdefibrillators: Pacing Fast Ventricular Tachycardia Reduces Shock Therapies (PainFREE Rx II) trial results. Circulation 110, 2591-2596

Whang W, Mittleman MA, Rich DQ, Wang PJ, Ruskin JN, Tofler GH, Muller JE, Albert CM (2004): Heart failure and the risk of shocks in patients with implantable cardioverter defibrillators: results from the Triggers Of Ventricular Arrhythmias (TOVA) study. Circulation 109, 1386-1391 
Wijers SC, Kolk BYM, Tuinenburg AE, Doevendans PAF, Vos MA, Meine M (2013): Implementation of guidelines for implantable cardioverter-defibrillator therapy in clinical practice: Which patients do benefit? Neth Heart J 21, 274-283

Wilkoff BL, Ousdigian KT, Sterns LD, Wang ZJ, Wilson RD, Morgan JM (2006): A comparison of empiric to physician-tailored programming of implantable cardioverterdefibrillators: results from the prospective randomized multicenter EMPIRIC trial. $J$ Am Coll Cardiol $\underline{48}, 330-339$

Wilkoff BL, Williamson BD, Stern RS, Moore SL, Lu F, Lee SW, Birgersdotter-Green UM, Wathen MS, van Gelder IC, Heubner BM et al. (2008): Strategic programming of detection and therapy parameters in implantable cardioverter-defibrillators reduces shocks in primary prevention patients: results from the PREPARE (Primary Prevention Parameters Evaluation) study. J Am Coll Cardiol $\underline{52}, 541-550$

Zipes DP, Camm AJ, Borggrefe M, Buxton AE, Chaitman B, Fromer M, Gregoratos G, Klein G, Moss AJ, Myerburg RJ et al. (2006): ACC/AHA/ESC 2006 guidelines for management of patients with ventricular arrhythmias and the prevention of sudden cardiac death: a report of the American College of Cardiology/American Heart Association Task Force and the European Society of Cardiology Committee for Practice Guidelines (Writing Committee to Develop Guidelines for Management of Patients With Ventricular Arrhythmias and the Prevention of Sudden Cardiac Death). J Am Coll Cardiol $\underline{48}$, e247-346 


\section{Abkürzungsverzeichnis}

\begin{tabular}{|c|c|}
\hline Abb. & Abbildung \\
\hline ACE & angiotensin converting enzyme \\
\hline ACVB & aorto-coronarer Venen-Bypass \\
\hline $\mathrm{AF}$ & atrial fibrillation (Vorhofflimmern) \\
\hline ARVC & arrhythmogene rechtsventrikuläre Kardiomyopathie \\
\hline AT & atriale Tachykardie \\
\hline AT1 & Angiotensin-II-Rezeptor-Subtyp-1 \\
\hline ATP & antitachykardes Pacing (antitachykarde Stimulation) \\
\hline AV & atrioventrikulär \\
\hline AVID & Antiarrhythmics versus Implantable Defibrillators (Studie) \\
\hline BMI & Body-Mass-Index \\
\hline BNP & brain natriuretic peptide \\
\hline bpm & beats per minute (Schläge pro Minute) \\
\hline CARE-HF & Cardiac Resynchronization - Heart Failure (Studie) \\
\hline CASCADE & Cardiac Arrest in Seattle: Conventional Versus Amiodarone Drug Evaluation (Studie) \\
\hline CASH & Cardiac Arrest Study Hamburg (Studie) \\
\hline $\mathrm{Cl}$ & Konfidenzintervall \\
\hline CIDS & Canadian implantable defibrillator study (Studie) \\
\hline COMPANION & Comparison of Medical Therapy, Pacing, and Defibrillation in Heart Failure (Studie) \\
\hline COPD & chronisch obstruktive Lungenerkrankung \\
\hline CRT-D & kardiale Resynchronisationstherapie plus Defibrillator \\
\hline DCM & dilatative Kardiomyopathie \\
\hline DEFINITE & Defibrillators in Non-Ischemic Cardiomyopathy Treatment Evaluation (Studie) \\
\hline DFT & defibrillation treshold (Defibrillationsschwelle) \\
\hline DINAMIT & Defibrillator in Acute Myocardial Infarction Trial (Studie) \\
\hline EGM & (intrakardiales) Elektrogramm \\
\hline EKG & Elektrokardiogramm \\
\hline \multirow[t]{2}{*}{ EMPIRIC } & Comparison of Empiric to Physician-Tailored Programming of Implantable \\
\hline & Cardioverter-Defibrillators (Studie) \\
\hline EPU & elektrophysiologische Untersuchung \\
\hline FVT & fast ventricular tachycardia (schnelle ventrikuläre Tachykardie) \\
\hline $\mathrm{HR}$ & hazard ratio \\
\hline ICD & implantierbarer Kardioverter-Defibrillator \\
\hline KHK & koronare Herzkrankheit \\
\hline LVEF & linksventrikuläre Ejektionsfraktion \\
\hline MADIT & Multicenter Automatic Defibrillator Implantation Trial (Studie) \\
\hline MADIT-II & Multicenter Automatic Defibrillator Implantation Trial II (Studie) \\
\hline MADIT-CRT & Multicenter Automatic Defibrillator Implantation Trial with Cardiac Resynchronization \\
\hline & Therapy (Studie) \\
\hline
\end{tabular}


MADIT-RIT Multicenter Automatic Defibrillator Implantation Trial - Reduce Inappropriate Therapy (Studie)

$\mathrm{mg} / \mathrm{dl} \quad$ Milligramm je Deziliter

$\mathrm{mm} \mathrm{Hg} \quad$ Millimeter Quecksilbersäule

ms Millisekunden

MUSTT Multicenter Unsustained Tachycardia Trial (Studie)

NID number of intervals detected

NYHA New York Heart Association

OPTIC Optimal Pharmacological Therapy in Cardioverter Defibrillator Patients (Studie)

p-Wert statistischer Signifikanzwert

PainFREE Pacing Fast VT Reduces Shock Therapies (Studie)

$\mathrm{PCl}$ perkutane Koronarintervention

$\mathrm{pg} / \mathrm{ml} \quad$ Pikogramm je Milliliter

PREPARE Primary Prevention Parameters Evaluation (Studie)

PROFIT Prospective Analysis of Risk Factors for appropriate ICD Therapy (Studie)

QALY quality adjusted life year

SCD-HeFT Sudden Cardiac Death in Heart Failure Trial (Studie)

SVT supraventrikuläre Tachykardie

TOVA Triggers Of Ventricular Arrhythmias (Studie)

$\mathrm{VF}$ ventricular fibrillation (Kammerflimmern)

VT ventrikuläre Tachykardie

z.B. zum Beispiel

Z.n. Zustand nach 


\section{Tabellenverzeichnis}

Tabelle 1: Kardiale Grunderkrankung $\quad 16$

Tabelle 2: Grundrhythmus bei Erstimplantation $\quad 16$

Tabelle 3: Funktionsbestimmung der LVEF 17

Tabelle 4: (Operative) Interventionen $\quad 17$

Tabelle 5: Nicht kardiale Grunderkrankungen $\quad 18$

Tabelle 6: Kardiovaskuläre Risikofaktoren $\quad 18$

Tabelle 7: Medikation bei ICD-Implantation $\quad 20$

Tabelle 8: Klinische Präsentation $\quad 20$

Tabelle 9: ICD-Implantationsindikation 21

Tabelle 10: Gerätetyp bei Erstimplantation $\quad 21$

Tabelle 11: Allgemeine ICD-Therapie 23

Tabelle 12: Appropriate ICD-Therapie 23

Tabelle 13: Inappropriate ICD-Therapie $\quad 24$

Tabelle 14: Ursachen der inappropriaten ICD-Therapie 24

Tabelle 15: Univariate Cox-Regressionsanalyse für appropriate Schocks 27

Tabelle 16: Multivariates Cox-Regressionsmodell für appropriate Schocks 28

Tabelle 17: Univariate Cox-Regressionsanalyse für inappropriate Schocks 31

Tabelle 18: Multivariates Cox-Regressionsmodell für inappropriate Schocks 32

Tabelle 19: Univariate Cox-Regressionsanalyse für Tod ohne vorherige $\begin{array}{ll}\text { appropriate Therapie } & 34\end{array}$

Tabelle 20: Multivariates Cox-Regressionsmodell für Tod ohne vorherige appropriate Therapie 


\section{Abbildungsverzeichnis}

Abbildung 1: Röntgenbild (p.a. Strahlengang) $\quad 8$

Abbildung 2: Röntgenbild (seitlicher Strahlengang) 8

Abbildung 3: ATP - Antitachykardes Pacing (EGM-Ausdruck) 11

Abbildung 4: Appropriater Schock für VT (EGM-Ausdruck) 12

Abbildung 5: Alter bei Erstimplantation des ICD $\quad 15$

Abbildung 6: Linksventrikuläre Ejektionsfraktion (LVEF)

Abbildung 7: Grad der Herzinsuffizienz (NYHA-Klassifikation) 19

Abbildung 8: Hersteller der Geräte 22

Abbildung 9: Auftreten des ersten ICD-Schocks $\quad 25$

Abbildung 10: Mortalität im Nachuntersuchungszeitraum 26

Abbildung 11: Appropriater Schock (Geschlecht) 29

Abbildung 12: Appropriater Schock (Primärprophylaxe) 29

Abbildung 13: Appropriater Schock (Amiodaroneinnahme) 30

Abbildung 14: Inappropriater Schock (Vorhofflimmern) 32

Abbildung 15: Inappropriater Schock (Implantationsalter) 33

Abbildung 16: Tod ohne vorherige appropriate Therapie (Implantationsalter) 35

Abbildung 17: Tod ohne vorherige appropriate Therapie (NYHA) 36

Abbildung 18: Tod ohne vorherige appropriate Therapie (Diuretika) 36

Quellennachweis Abbildungen 1 - 4: ICD-Ambulanz, Universitätsmedizin Göttingen 


\section{Danksagung}

Mein besonderer Dank gilt Herrn Professor Dr. med. Markus Zabel für die freundliche Überlassung des Themas sowie die fachliche Unterstützung und Betreuung in jeder Phase der Erstellung der vorliegenden Arbeit.

Ebenso danke ich Herrn Dr. med. Marc Dorenkamp für die Betreuung vor allem in der Anfangsphase der Arbeit, der ermöglichten Teilnahme an ICD-Implantationen und elektrophysiologischen Untersuchungen sowie der Unterstützung bei der Datenaufnahme.

Weiterhin danke ich Frau Sabine Busse für die Erklärungen zur praktischen Arbeit in der ICD-Ambulanz und die Einführung in die Programmierung der Aggregate.

Außerdem bin ich dankbar für die Beratungsangebote des Instituts für Medizinische Statistik der Universität Göttingen und die Erläuterungen zur statistischen Analyse. 